\author{
Universidade De SÃo Paulo \\ InSTITUTO DE FÍSICA
}

\title{
Formulação Cinética para Cosmologias com Criação de Matéria e Aplicações
}

\author{
IURI BARANOV PEREIRA RAYMUNDO
}

Orientador: Prof. Dr. José Ademir Sales de Lima

Tese de doutorado apresentada ao Instituto de Física para a obtenção do título de Doutor em Ciências

Banca Examinadora:

Prof. Dr. José Ademir Sales de Lima - IAG-USP.

Prof. Dr. Júlio Fabris - UFES.

Prof. Dr. Winfried Zimdahl - UFES.

Prof. Dr. Carlos Molina - EACH-USP.

Profa. Dra. Renata Funchal - IFUSP.

São Paulo 


\section{Agradecimentos}

Agradeço ao professor José Ademir Sales de Lima pela sugestão do tema desta tese e pelas discussões.

A todos do nosso grupo de pesquisa: David, Diego, Felipe, George, João Vital, José Fernando, Leila, Leonardo, Michel, Sílvio e Vinícius. Em especial ao professor Gary Steigman pelas ricas discussões.

A todos funcionários do IFUSP e do IAG. Ao Departamento de Física Geral do IFUSP pela infra-estrutura concedida.

Agradeço à minha mãe Tamara e ao meu pai Paulo, pela dedicação, apoio e incentivo incondicionais. Ao meu irmão Carlos e à minha sobrinha Larissa, que são fonte de inspiração e grande influência.

À minha namorada Claire, pela companhia.

À CAPES e CNPq pelo suporte financeiro. 


\section{Resumo}

Nesta tese, estudamos cosmologias com criação de matéria como alternativa ao modelo $\Lambda$ CDM. Generalizamos a equação de Boltzmann relativística com um termo de produção de partículas fenomenológico, de tal forma que a equação resultante seja capaz de reobter os resultados da termodinâmica de não-equilíbrio das equações de balanço e evolução de temperatura.

Após obter o termo correto para a equação generalizada de Boltzmann, investigamos como o formalismo proposto altera a equação de evolução de relíquias cósmicas na presença de criação gravitacional de partículas. 


\section{Abstract}

In this thesis, we study matter creation cosmologies as an alternative to the $\Lambda \mathrm{CDM}$ model. We generalize the relativistic Boltzmann equation with a phenomenological particle production term, in such a way that the resulting equation will be able to reproduce the non-equilibrium thermodynamics results of the balance equations and temperature evolution law.

After obtaining the correct term to the generalized Boltzmann equation, we investigate how the proposed formalism changes the cosmic relic evolution equation in the presence of gravitational particle creation. 


\section{Convenções}

- Adotaremos o sistema de unidades naturais em que $c=k=h=1$. Quando for conveniente, reescreveremos as constantes explicitamente.

- Índices gregos variam de 0 a 3 e índices latinos variam de 1 a 3.

- Índices repetidos significam soma (convenção de Einstein).

- Assinatura da métrica: $(+,-,-,-)$.

- A métrica de Minkowski é

$$
\eta_{\mu \nu}=\operatorname{diag}(1,-1,-1,-1)
$$

- A derivada parcial é $\frac{\partial \phi}{\partial x^{\mu}} \equiv \partial_{\mu} \phi \equiv \phi,{ }_{\mu}$.

- A derivada covariante de um campo tensorial é

$$
\nabla_{\mu} T_{\beta \cdots}^{\alpha \cdots}=\partial_{\mu} T_{\beta \cdots}^{\alpha \cdots}+\Gamma_{\gamma \mu}^{\alpha} T_{\beta \cdots}^{\gamma \cdots}+\cdots-\Gamma_{\beta \mu}^{\gamma} T_{\gamma \cdots}^{\alpha \cdots}-\cdots
$$




\section{Lista de Figuras}

1.1 Expansão do espaço-tempo. . . . . . . . . . . . . . . . . . 8

1.2 Gráfico obtido por Hubble para sua lei $v=H_{0} d$. Figura retirada de [1] . . 9

1.3 Raios de luz sucessivos de uma galáxia em $P$ até um observador $O$. . . 10

1.4 Densidades de energia de diferentes componentes em função do fator de escala $a \ldots \ldots \ldots \ldots \ldots$. . . . . . . . . . . . . . . 19

1.5 Fatores de escala para diferentes modelos cosmológicos. . . . . . . . . . . 21

1.6 Influência de $\Lambda$ na idade total do Universo. . . . . . . . . . . . . . 23

1.7 Parâmetro de desaceleração para o modelo $\Lambda$ CDM em função do redshift. . 24

1.8 Distância de luminosidade para diferentes parâmetros. . . . . . . . . . . 26

1.9 Evidência da expansão acelerada do Universo. Figura retirada de [2] . . . . 26

2.1 Solução da equação de evolução para diferentes valores de $\lambda . \ldots$. . . . . 47

3.1 Fator de escala para $\gamma=0.3$ e dois valores distintos de $\beta$. Os resultados são comparados com o modelo Einstein-de Sitter. . . . . . . . . . . . . 54

3.2 Idade do Universo em função de $\gamma$ para alguns valores de $\beta$. A linha verde representa o limite inferior para a idade do Universo. . . . . . . . . . . . 55

3.3 Parâmetro de desaceleração para diferentes valores de $\beta$ e $\gamma$. . . . . . . 56

3.4 Vínculos obtidos através de análise de Supernovas do tipo Ia. Figura retirada de $[3] . \ldots \ldots \ldots \ldots$

3.5 Taxa de criação de matéria escura e parâmetro de Hubble em função do

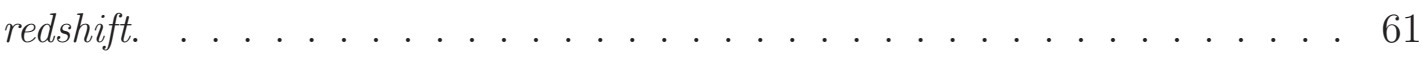

3.6 Quociente entre taxa de criação de matéria e parâmetro de Hubble, parâmetro de desaceleração e quociente entre pressão de criação e densidade de matéria criada em função do redshift. . . . . . . . . . . . . . 62 
3.7 Quantidade de matéria criada por unidade de volume de Hubble por ano em função do redshift. . . . . . . . . . . . . . . . . . . . . . . . 62

5.1 Soluções da nova equação de evolução para diferentes valores de $\beta$. O parâmetro $\lambda$ foi fixado em $\lambda=10^{13}$. Mais detalhes no texto. . . . . . . . . 73

5.2 Diferença percentual $\Delta Y=\frac{Y-Y_{\text {usual }}}{Y_{\text {usual }}}$ para diferentes valores de $\beta, \lambda=10^{13} . \quad 76$

5.3 Parâmetro de densidade de relíquias $\Omega_{\chi} h^{2}$ como função de $<\sigma v>$. . . . 76

$5.4<\sigma v>$ como função de $\beta$ para o best-fit do experimento Planck $\Omega_{\chi} h^{2}=$

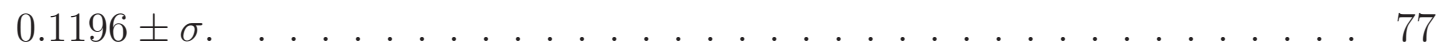

$5.5 \Omega_{\chi} h^{2}$ em função de $u$ para o modelo LJO. . . . . . . . . . . . . . . . . . 80

5.6 Diferença percentual entre modelo LJO para o caso usual sem criação de matéria em função de $u$. . . . . . . . . . . . . . . . . . . . . . . . 80 


\section{Sumário}

Agradecimentos $\quad$ i

Resumo

Abstract

Convenções $\quad$ iv

Lista de Figuras $\quad$ v

$\begin{array}{ll}\text { Introdução } & 1\end{array}$

1 Cosmologia 5

1.1 Métrica de Friedmann-Lemaître-Robertson-Walker e Lei de Hubble . . . . 6

1.2 Redshift Cosmológico . . . . . . . . . . . . . . . . . . . 8

1.3 Tensor energia-momento de um Fluido Perfeito . . . . . . . . . . . . . . 11

1.4 Dinâmica Cosmológica e Equações de Friedmann ......... 13

1.5 Cosmologias do tipo FLRW . . . . . . . . . . . . . . . 17

1.6 Distância de Luminosidade e Aceleração do Universo . . . . . . . . . . . . . 25

2 Teoria Cinética Relativística, Equilíbrio na Métrica FLRW e $\begin{array}{lr}\text { Abundâncias Cósmicas } & 28\end{array}$

2.1 Equação de Boltzmann Relativística . . . . . . . . . . . . . . . . 30

2.2 Equação de Transferência e Invariantes de Soma . . . . . . . . . . . . . . . 33

2.3 Entropia e Função Distribuição de Equilíbrio . . . . . . . . . . . . . . . . . 34

2.4 Equação de Boltzmann na métrica FLRW . . . . . . . . . . . . . . . 40

2.4.1 Equações de Balanço . . . . . . . . . . . . . . . . . . . . . . . 41

2.4.2 Lei de Evolução da Temperatura . . . . . . . . . . . . . . . . . . . 44 
2.4.3 Equação de Evolução de Relíquias Cósmicas . . . . . . . . . . . . . 45

3 Criação Gravitacional de Partículas: Formulação Termodinâmica e Cos$\begin{array}{ll}\text { mologia } & 48\end{array}$

3.1 Termodinâmica do Modelo . . . . . . . . . . . . . . . . . . . 50

3.2 Cosmologia com Criação de Matéria Escura . . . . . . . . . . . . . . . 53

3.3 Criação de Matéria $\times \Lambda$ CDM: O Modelo LJO . . . . . . . . . . . . 56

3.3.1 Redshift de Transição e Vínculos de Supernova . . . . . . . . . . . . 59

4 Criação Gravitacional de Partículas: Formulação Cinética $\quad 64$

4.1 Equações de Balanço . . . . . . . . . . . . . . . . . . 67

4.2 Lei de Temperatura . . . . . . . . . . . . . . . . . . . . . 69

5 Nova Equação de Relíquias Cósmicas $\quad 72$

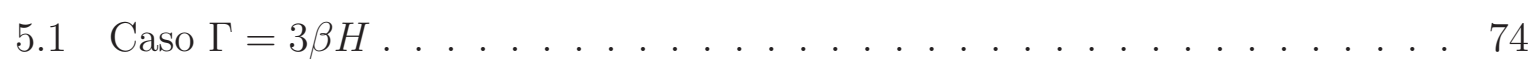

5.2 Produção de WIMPs no Modelo LJO . . . . . . . . . . . . . . . . 78

6 Conclusão e Perspectivas $\quad 81$

A Dedução alternativa da Equação de Boltzmann $\quad 84$

B Dedução do termo colisional da equação de evolução $\quad 87$

C Equação de Boltzmann em momentos comóveis $\quad 90$

$\begin{array}{ll}\text { Referências Bibliográficas } & 93\end{array}$ 


\section{Introdução}

A Cosmologia atual encontra na Teoria da Relatividade Geral (TRG)[4], desenvolvida por Einstein em 1916, um arcabouço teórico jamais disponível para o estudo completo do Universo. Através da TRG e do desenvolvimento de equipamentos e técnicas observacionais, a Cosmologia evoluiu de meras especulações filosóficas para uma ciência consolidada, com atividades teóricas e experimentais.

A primeira consequência cosmológica das equações de campo de Einstein a foi de que o Universo poderia ter uma dinâmica. À época, era senso comum que o Universo era estático. Essa discrepância fez com que Einstein introduzisse um novo termo em suas equações, a chamada constante cosmológica [5].

Na contramão do pensamento vigente, Alexander Friedmann publicou em 1922 uma série de equações baseadas nas equações de Einstein que previam a expansão do Universo com curvatura positiva [6]. Em 1924, Friedmann obteve soluções expansionistas com curvatura negativa [7]. De forma independente, Georges Lemaître também obteve soluções expansionistas [8]. Contudo, seu trabalho original ficou desconhecido por muitos anos. As soluções expansionistas ficaram desacreditados até 1929, quando Edwin Hubble constatou que a luz proveniente das galáxias apresentavam um desvio para o vermelho, o redshift [1]. Hubble obteve uma relação empírica entre a velocidade de afastamento das galáxias e a distância até nós. Descartando a possibilidade de que esse movimento fosse devido a velocidades peculiares das galáxias, essa descoberta fez com que se abandonasse a ideia de um Universo estático. Nascia a teoria do Big Bang: em algum momento no passado o Universo passou a expandir até os dias de hoje.

A expansão cósmica observada por Hubble é um dos três grandes pilares observacionais que sustentam a teoria do Big Bang. Os outros são a predição da abundância de elementos leves e a observação, por Penzias e Wilson, da radiação cósmica de fundo em 1965, prevista por Gamow em 1948. 
Apesar dos sucessos da teoria do Big Bang, veremos adiante que a Cosmologia atual enfrenta dois grandes problemas. Evidências observacionais garantem que existe mais matéria do que a matéria bariônica comum, o chamado problema da matéria escura. Em diversas escalas, a presença da matéria escura é notada: desde curvas de rotação de galáxias até observações de lentes gravitacionais em aglomerados de galáxias. Alguns testes cosmológicos também confirmam a existência da matéria escura, como, por exemplo, análise do espectro de potência da radiação cósmica de fundo. A expansão cósmica, ao contrário do que era de se esperar, acontece de forma acelerada. Esse fato, observado por dois grupos independentes [9, 10], somado a outras evidências como, por exemplo, a baixa idade do Universo para modelos com apenas matéria, fez com que se sugerisse a existência de uma nova componente na natureza, a chamada energia escura. Uma revisão sobre os problemas da matéria e energia escuras pode ser encontrada em [11, 12].

Dentro do contexto da TRG, o ingrediente fundamental para que haja uma expansão acelerada do Universo é uma pressão suficientemente negativa capaz de alterar o sinal da equação de aceleração do fator de escala. Essa equação é obtida através de uma composição das duas equações de Friedmann. Diferentes mecanismos podem explicar tal pressão negativa: uma constante cosmológica, um campo escalar, existência de fluidos exóticos etc. Um dos candidatos mais estudados é a energia associada ao vácuo. Para que o vácuo seja o mesmo para todos observadores, ele precisa ter uma equação de estado da forma $p=-\rho$, em que $\rho$ é sua densidade de energia. O modelo cosmológico mais usual é o chamado $\Lambda$ CDM. Nele, além da matéria escura, temos outra nova componente, a constante cosmológica $\Lambda$, que usualmente está associada à energia do vácuo, e é a responsável pela aceleração da expansão do Universo. Contudo, a divergência entre os valores observados e calculados pela teoria quântica de campo para $\Lambda$ pode chegar a 120 ordens de grandeza; isso caracteriza uma das maiores discrepâncias entre teoria e observação da Física e é chamada problema da constante cosmológica.

Como veremos, a contribuição energética da matéria escura é da mesma ordem da contribuição da energia escura apenas hoje. Esse é outro problema enfrentado pelo modelo $\Lambda \mathrm{CDM}$ e é chamado de problema da coincidência cósmica. Por que as densidades de matéria e de energia escura são da mesma ordem de grandeza hoje?

Com as duas questões levantadas acima (problema da constante cosmológica e da coincidência cósmica) ainda em aberto, grupos de pesquisa investigam modelos cosmológicos 
alternativos ao $\Lambda \mathrm{CDM}$ [13], dentre os quais destacamos os chamados modelos cosmológicos dissipativos. O primeiro modelo dissipativo, proposto por [14], sugere que a criação de matéria na era de Planck poderia ser modelada como uma viscosidade volumar. A segunda alternativa, seriam os modelos cosmológicos com criação gravitacional de partículas, proposto inicialmente por [15]. Como mostrado por [16], os dois modelos possuem características dinâmicas equivalentes, porém com propriedades termodinâmicas completamente diferentes. Como veremos em capítulos posteriores, a criação de partículas à custa do campo gravitacional gera uma pressão negativa, fundamental para a expansão acelerada do Universo. Esta tese, além de reunir informações sobre o modelo cosmológico de criação de partículas, tem como objetivo propor formulação cinética para a produção de partículas a partir da equação de Boltzmann. A estrutura da tese é detalhada a seguir.

No Capítulo 1 faremos uma revisão da Cosmologia moderna. Será discutido o conceito de métrica e apresentaremos a métrica de Friedmann-Lemaître-Robertson-Walker (FLRW). Lançando mão da TRG, obteremos as equações básicas da Cosmologia. Estudaremos modelos cosmológicos simples e, ao final do capítulo, introduziremos os problemas da matéria e energia escuras.

No Capítulo 2 é apresentada a teoria cinética relativística. Serão discutidos conceitos básicos e será obtida a equação de Boltzmann relativística. Posteriormente, aplicaremos a métrica de FLRW na equação de Boltzmann; a partir desta última, recuperaremos alguns resultados da termodinâmica, a saber: equações de balanço em equilíbrio e lei de evolução da temperatura. Ao final do capítulo, discutiremos como se obtém a equação de evolução para relíquias cósmicas.

No Capítulo 3 discutiremos a termodinâmica de processos de não-equilíbrio de criação de partículas no contexto da Cosmologia. Veremos que a presença de tal processo resulta no surgimento de uma pressão negativa, capaz de explicar a expansão acelerada do Universo. Faremos uma revisão de modelos cosmológicos com criação de matéria, dando ênfase, ao final, ao modelo LJO [3], que é um boa alternativa ao $\Lambda$ CDM.

Por fim, no Capítulo 4 apresentaremos nossos primeiros resultados originais, que podem ser obtidos na íntegra em [17]. Proporemos um modelo cinético capaz de explicar os resultados termodinâmicos de não-equilíbrio (equações de balanço e lei de evolução de temperatura) do processo de criação gravitacional de partículas. Esse novo termo se traduz numa nova equação de Boltzmann. 
Como aplicação direta do mecanismo desenvolvido, no Capítulo 5 estudaremos como a nova equação de Boltzmann afeta a evolução de relíquias cósmicas para alguns modelos. Os resultados originais obtidos nesse capítulo estão disponíveis em [18].

No Capítulo 6 é apresentado um resumo dos resultados obtidos e uma conclusão. Também serão mencionadas perspectivas para trabalhos futuros. 


\section{Capítulo 1}

\section{Cosmologia}

Em 1916, Einstein publica a generalização da sua teoria da relatividade restrita, a teoria da Relatividade Geral (RG) [4]. Dentre as variadas aplicações da RG, é utilizando-a como base teórica para descrever o Universo que nasce a Cosmologia moderna. Em 1917, Einstein publica uma alteração em suas equações de campo da RG [5]. Para explicar um Universo estático, Einstein incluiu às suas equações uma nova constante, a constante cosmológica.

Treze anos mais tarde, Hubble [1] muda a visão que os cientistas tinham da dinâmica do Universo. Ao contrário do que se imaginava, o Universo está em expansão. Essa observação é um dos três pilares observacionais da Cosmologia. Os outros são: detecção da radiação cósmica de fundo e abundância de elementos leves.

Junto com os três pilares observacionais e a teoria da RG, o chamado Modelo Padrão da Cosmologia (MPC) lança mão de uma importante hipótese, chamada de princípio cosmológico: em larga escala o Universo é homogêneo (todos os pontos são equivalentes) e isotrópico (todas as direções são equivalentes). Por larga escala queremos dizer distâncias superiores a $100 \mathrm{Mpc}$.

Antes de o MPC ser discutido com mais detalhes, faremos uma revisão das ferramentas da RG que serão úteis ao longo do texto. Para uma abordagem mais detalhada ver $[19,20,21,22,23,24]$. 


\subsection{Métrica}

\section{Friedmann-Lemaître-Robertson-Walker e Lei de Hubble}

Na RG, o espaço e o tempo são tratados como dimensões de uma estrutura quadridimensional (uma dimensão de tempo e três de espaço) chamada de espaço-tempo. Matematicamente, o que caracteriza o espaço-tempo é sua métrica.

Em um espaço-tempo quadridimensional, a distância entre dois eventos é chamada de intervalo, ds. Esse intervalo é representado pelo elemento de linha invariante

$$
d s^{2}=g_{\mu \nu} d x^{\mu} d x^{\nu}
$$

em que $g_{\mu \nu}$ é a métrica e os índices variam de 0 a 3.

Partículas livres no espaço-tempo percorrem trajetórias ditas geodésicas e obedecem à equação de geodésica

$$
\frac{d^{2} x^{\mu}}{d \lambda^{2}}+\Gamma_{\alpha \beta}^{\mu} \frac{d x^{\alpha}}{d \lambda} \frac{d x^{\beta}}{d \lambda}=0
$$

em que $\lambda$ é um parâmetro afim. Os símbolos $\Gamma_{\alpha \beta}^{\mu}$ são chamados de símbolos de Christoffel de segundo tipo ou conexões. Eles podem ser obtidos através da métrica pela relação

$$
\Gamma_{\alpha \beta}^{\mu}=\frac{1}{2} g^{\mu \nu}\left(\partial_{\beta} g_{\alpha \nu}+\partial_{\alpha} g_{\beta \nu}-\partial_{\nu} g_{\alpha \beta}\right)
$$

Em coordenadas cartesianas, as conexões são nulas (as componentes do tensor métrico são constantes e, portanto, suas derivadas em relação às coordenadas são nulas). Vemos da equação de geodésica (1.2) que as soluções para o movimento de uma partícula livre nesse sistema de coordenadas são as de uma reta.

A métrica que descreve um Universo expansionista homogêneo e isotrópico é a métrica de Friedmann-Lemaître-Robertson-Walker (FLRW) e é dada por [19]

$$
d s^{2}=d t^{2}-a^{2}(t)\left[\frac{d r^{2}}{1-\kappa r^{2}}+r^{2} d \theta^{2}+r^{2} \sin ^{2} \theta d \phi^{2}\right]
$$

em que $(t, r, \theta, \phi)$ são coordenadas comóveis, $\kappa=0, \pm 1$ é o parâmetro de curvatura. $a(t)$ é o fator de escala, uma função dependente do tempo que contém as informações da 
expansão do espaço.

Imaginemos uma galáxia distante de nós com coordenadas $(r, \theta, \phi)$. A distância da galáxia até nós, em um tempo fixo $t$, lembrando que as coordenadas $(\theta, \phi)$ não variam, é dada por

$$
d(r, t)=a(t) \int_{0}^{r} \frac{d r}{\sqrt{1-\kappa r^{2}}}=a(t) \times\left\{\begin{array}{ll}
\sin ^{-1} r & \text { para } \kappa=+1 \\
r & \text { para } \kappa=0 \\
\sinh ^{-1} r & \text { para } \kappa=-1
\end{array}=a(t) f(r)\right.
$$

A quantidade expressa pela integral é a distância coordenada, que depende apenas da coordenada comóvel $r$ da galáxia e não depende de $t$. A distância física $d(r, t)$ entre nós e a galáxia é modulada através da evolução temporal do fator de escala $a(t)$. Se $a(t)$ aumenta com o tempo, a distância física entre nós e a galáxia aumenta e a galáxia se afasta de nós. Caso $a(t)$ diminua com o tempo, a distância física também diminui e a galáxia se aproxima de nós.

Para entender melhor o que é uma coordenada comóvel e como a distância física varia com o tempo, observemos a Figura 1.1. No lado esquerdo, mostramos uma configuração inicial de duas galáxias, digamos, nós (galáxia vermelha) e uma galáxia distante (galáxia azul). Cada galáxia ocupa uma posição dada pelo par coordenado $\left(X^{1}, X^{2}\right)$ e a distância física inicial é $d i$. Com a expansão do espaço, a distância física entre as galáxias aumenta, $d f>d i$. Mas as coordenadas que cada uma ocupa permanecem as mesmas. Essas coordenadas, que não mudam com a expansão do espaço, são as coordenadas comóveis.

Se derivarmos a expressão (1.5) com relação ao tempo obtemos

$$
\dot{d}=\dot{a}(t) f(r)=\frac{\dot{a}(t)}{a(t)} d
$$

lembrando que $f(r)$ não depende da coordenada $t$ e que $f(r)=\frac{d}{a}$. Ou seja, a expressão para a velocidade de afastamento das galáxias pode ser escrita como

$$
v=H(t) d
$$

em que $H(t) \equiv \frac{\dot{a}(t)}{a(t)}$ é chamado parâmetro de Hubble. Em particular, para o tempo de hoje, $H_{0}=H\left(t_{0}\right)$ é a constante de Hubble.

Em 1929, Hubble observou que as galáxias estavam se afastando de nós com uma 


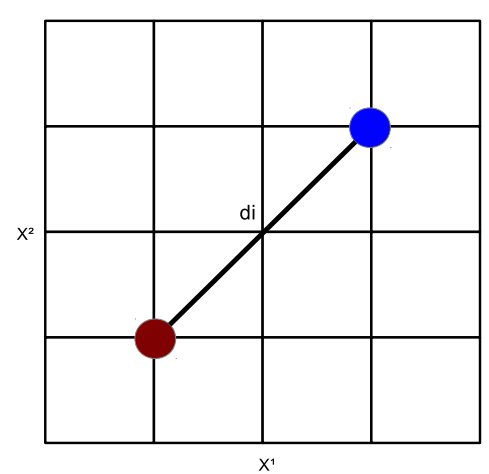

(a) Configuração inicial do espaço-tempo

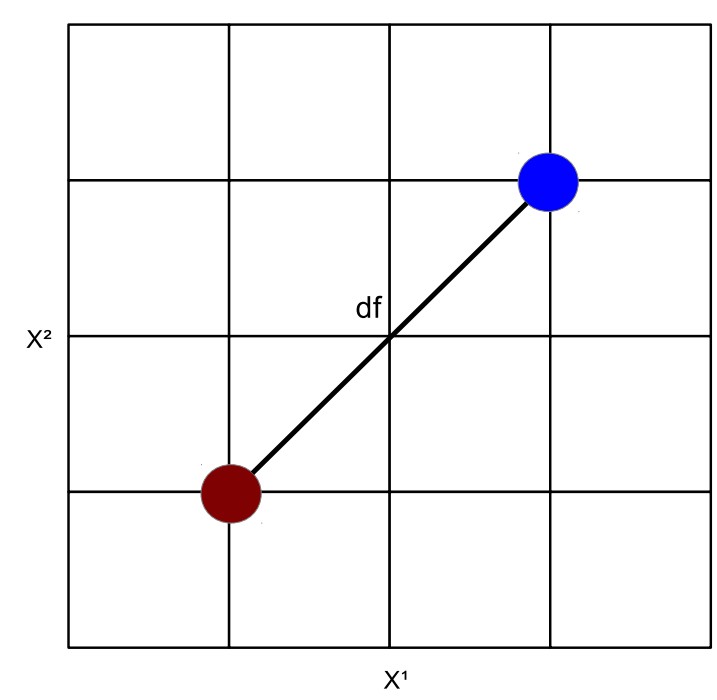

(b) Configuração do espaçotempo após um intervalo de tempo

Figura 1.1: Expansão do espaço-tempo.

velocidade que aumentava à medida que a distância aumentava. Essa relação empírica obtida por Hubble é a mesma obtida em (1.7) quando calculamos para $t=t_{0}$.

$$
v=H_{0} d
$$

é a lei de Hubble. Na Figura 1.2 é mostrado o gráfico original obtido por Hubble [1].

\section{$1.2 \quad$ Redshift Cosmológico}

Em Astronomia, os dados observacionais são obtidos através da radiação que os objetos emitem e que chega aos nossos telescópios. É preciso saber como a luz se propaga na métrica FLRW.

Consideremos que estamos observando uma galáxia posicionada em um ponto $P$, com coordenadas $\left(r_{1}, \theta_{1}, \phi_{1}\right)$, e que estamos na origem $O$, com coordenadas $(0,0,0)$. As coor- 


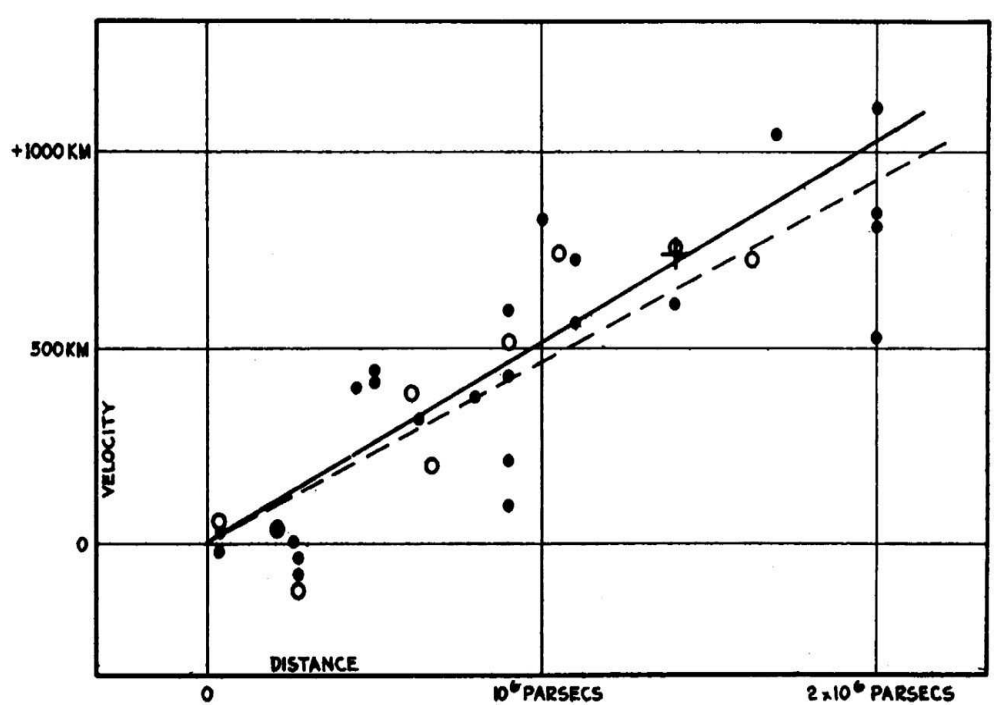

Figura 1.2: Gráfico obtido por Hubble para sua lei $v=H_{0} d$. Figura retirada de [1].

denadas angulares da luz emitida pela galáxia não mudam, isto significa que $d \theta=d \phi=0$. Partículas sem massa, como a luz, percorrem geodésicas nulas, ou seja, $d s^{2}=0$. Com essas informações, podemos utilizar a métrica de FLRW para escrevermos

$$
\frac{d t}{a(t)}= \pm \frac{d r}{\sqrt{1-\kappa r^{2}}}
$$

em que o sinal "-" designa a luz que chega ao observador e o sinal "+" a luz que se afasta do observador.

Podemos relacionar o redshift de um objeto, nesse caso, da galáxia em $P$, com o fator de escala na época em que o objeto emitiu a luz agora observada. Para isso, consideremos que a galáxia em $r_{1}$ emita um raio de luz no instante $t=t_{1}$. Esse raio de luz viaja sob a geodésica nula até ser observado por nós, em $r=0$ e em um instante $t=t_{0}$. Integrando a expressão anterior, obtemos

$$
\int_{t_{1}}^{t_{0}} \frac{d t}{a(t)}=-\int_{r_{1}}^{0} \frac{d r}{\sqrt{1-\kappa r^{2}}}
$$

O termo da esquerda da expressão acima depende apenas de $t$, enquanto que o termo da direita apenas de $r$. Analisaremos agora a emissão de dois raios sucessivos emitidos pela galáxia. O primeiro é emitido em $t=t_{1}$ e recebido em $t=t_{0}$. O segundo raio parte no instante $t=t_{1}+d t_{1}$ que será observado em $t=t_{0}+d t_{0}$. A Figura 1.3 ilustra melhor a situação. Para o segundo raio temos 


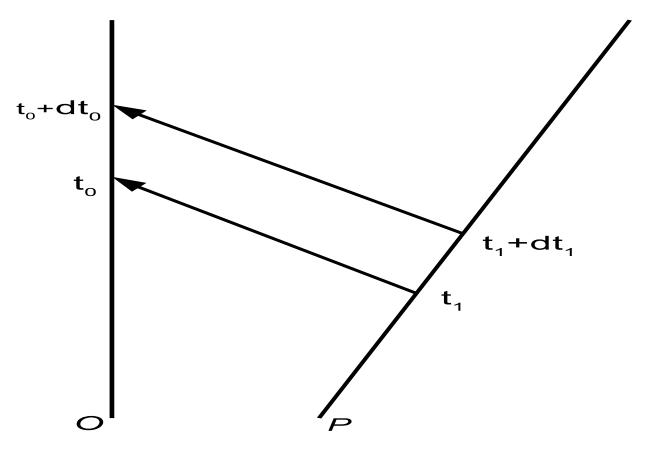

Figura 1.3: Raios de luz sucessivos de uma galáxia em $P$ até um observador $O$.

$$
\int_{t_{1}+d t_{1}}^{t_{0}+d t_{0}} \frac{d t}{a(t)}=-\int_{r_{1}}^{0} \frac{d r}{\sqrt{1-\kappa r^{2}}}
$$

pois o ponto $P$ está localizado em uma coordenada comóvel. Portanto, podemos igualar as expressões (1.10) e (1.11)

$$
\int_{t_{1}}^{t_{0}} \frac{d t}{a(t)}=\int_{t_{1}+d t_{1}}^{t_{0}+d t_{0}} \frac{d t}{a(t)}
$$

Manipularemos estas integrais da seguinte maneira:

$$
\int_{t_{1}}^{t_{0}} \frac{d t}{a(t)}=\int_{t_{1}}^{t_{1}+d t_{1}} \frac{d t}{a(t)}+\int_{t_{1}+d t_{1}}^{t_{0}} \frac{d t}{a(t)},
$$

e

$$
\int_{t_{1}+d t_{1}}^{t_{0}+d t_{0}} \frac{d t}{a(t)}=\int_{t_{1}+d t_{1}}^{t_{0}} \frac{d t}{a(t)}+\int_{t_{0}}^{t_{0}+d t_{0}} \frac{d t}{a(t)},
$$

Com isso, ficamos com

$$
\int_{t_{1}}^{t_{1}+d t_{1}} \frac{d t}{a(t)}=\int_{t_{0}}^{t_{0}+d t_{0}} \frac{d t}{a(t)} .
$$

Durante a emissão de sucessivos raios de luz pelo objeto, o Universo não expandiu muito. Ou seja, $a(t)$ pode ser considerado constante na expressão acima. Assim, podemos escrever da seguinte maneira:

$$
\frac{1}{a\left(t_{1}\right)} \int_{t_{1}}^{t_{1}+d t_{1}} d t=\frac{1}{a\left(t_{0}\right)} \int_{t_{0}}^{t_{0}+d t_{0}} d t
$$


$\mathrm{ou}$,

$$
\frac{d t_{1}}{a\left(t_{1}\right)}=\frac{d t_{0}}{a\left(t_{0}\right)}
$$

Da relação acima, podemos extrair um importante resultado. Para isso, lembramos que o comprimento de onda emitido é $\lambda_{1}=c / \nu_{1}$, em que $c$ é a velocidade da luz e $\nu_{1}$ é a frequência da onda emitida, que pode ser relacionada com $d t_{1}$, o período de emissão, por $d t_{1}=1 / \nu_{1}$. Dessa maneira, $d t_{1}=\lambda_{1} / c$. Da mesma forma, o comprimento de onda observado pode ser relacionado a $d t_{0}, d t_{0}=\lambda_{0} / c$. Com isso, temos

$$
\frac{\lambda_{1}}{a\left(t_{1}\right)}=\frac{\lambda_{0}}{a\left(t_{0}\right)}
$$

Podemos usar a definição de redshift,

$$
z=\frac{\lambda_{\text {observado }}-\lambda_{\text {emitido }}}{\lambda_{\text {emitido }}}
$$

para relacionar o fator de escala ao redshift

$$
1+z=\frac{a\left(t_{0}\right)}{a\left(t_{1}\right)}=\frac{1}{a\left(t_{1}\right)},
$$

em que usamos a convenção $a\left(t_{0}\right)=1$. Ao observar o redshift de um objeto, obtemos informações de como era o fator de escala na época de emissão do raio de luz. Como é obtido o fator de escala será discutido na seção seguinte.

\subsection{Tensor energia-momento de um Fluido Perfeito}

Um fluido perfeito é aquele em que um observador, movendo-se com a mesma velocidade $v$ do fluido, o enxerga como isotrópico. Portanto, no sistema de referência comóvel ao fluido, as componentes do tensor de energia-momento são

$$
\begin{aligned}
& T^{\prime 00}=\rho, \\
& T^{\prime i j}=p \delta^{i j}, \\
& T^{\prime 0 i}=T^{\prime i 0}=0,
\end{aligned}
$$

em que $\rho$ é a densidade de energia do fluido e $p$ é a pressão isotrópica. 
Denotaremos por $x^{\prime \mu}$ as coordenadas em um sistema de referência comóvel ao fluido, $K^{\prime}$ e $x^{\mu}$ as coordenadas em um sistema arbitrário $K$ em repouso. Os dois sistemas estão conectados pelas transformações de Lorentz

$$
\left[\begin{array}{l}
t^{\prime} \\
x^{\prime} \\
y^{\prime} \\
z^{\prime}
\end{array}\right]=\left[\begin{array}{cccc}
\gamma & -\gamma \beta_{x} & -\gamma \beta_{y} & -\gamma \beta_{z} \\
-\gamma \beta_{x} & 1+(\gamma-1) \frac{\beta_{x}^{2}}{\beta^{2}} & (\gamma-1) \frac{\beta_{x} \beta_{y}}{\beta^{2}} & (\gamma-1) \frac{\beta_{x} \beta_{z}}{\beta^{2}} \\
-\gamma \beta_{y} & (\gamma-1) \frac{\beta_{y} \beta_{x}}{\beta^{2}} & 1+(\gamma-1) \frac{\beta_{y}^{2}}{\beta^{2}} & (\gamma-1) \frac{\beta_{y} \beta_{z}}{\beta^{2}} \\
-\gamma \beta_{z} & (\gamma-1) \frac{\beta_{z} \beta_{x}}{\beta^{2}} & (\gamma-1) \frac{\beta_{z} \beta_{y}}{\beta^{2}} & 1+(\gamma-1) \frac{\beta_{z}^{2}}{\beta^{2}}
\end{array}\right]\left[\begin{array}{l}
t \\
x \\
y \\
z
\end{array}\right]
$$

como estamos adotando unidades naturais em que $c=1, \gamma \equiv\left[1-(v)^{2}\right]^{-1 / 2}$ e $\beta_{i}=v_{i}$. Ou de forma mais compacta

$$
x^{\prime \mu}=\Lambda(v)_{\nu}^{\mu} x^{\nu} .
$$

Em um sistema de referência arbitrário, o tensor de energia-momento é

$$
T^{\mu \nu}=\bar{\Lambda}_{\alpha}^{\mu} \bar{\Lambda}_{\beta}^{\mu} T^{\alpha \beta}
$$

em que $\bar{\Lambda}_{\nu}^{\mu}=\Lambda(-v)_{\nu}^{\mu}$ tem a mesma forma que (1.24) porém com o sinal da velocidade trocado.

As componentes do tensor no referencial $K$ são: $T^{00}=(\rho+p) \gamma^{2}-p, T^{i 0}=T^{0 i}=$ $(\rho+p) \gamma^{2} \beta^{i}$ e $T^{i j}=(\rho+p) \gamma^{2} \beta^{i} \beta^{j}+p \delta^{i j}$. Com auxílio da métrica de Minkowski (1) e em termos da quadrivelocidade do fluido medido pelo observador em $K, u^{\mu}=\left(\gamma, \gamma v^{i}\right)$, podemos escrever o tensor de energia-momento da seguinte forma tensorial:

$$
T^{\mu \nu}=(\rho+p) u^{\mu} u^{\nu}-p \eta^{\mu \nu}
$$

Utilizando o Princípio da Covariância Geral [19], escrevemos o tensor de energia-momento para um fluido perfeito na RG substituindo a métrica de Minkowski por uma métrica arbitrária

$$
T^{\mu \nu}=(\rho+p) u^{\mu} u^{\nu}-p g^{\mu \nu}
$$

Notamos que a construção do tensor de energia-momento feita acima é geral e vale para qualquer referencial. Em particular, para estudarmos a dinâmica do Universo, assumiremos um referencial comóvel ao fluido. Isso significa dizer que, à medida que o Universo 
expande, o fluido contido nele acompanha a expansão. Observadores que possuem velocidades peculiares desprezíveis em relação à velocidade de expansão, serão comóveis ao fluido. Matematicamente, temos que a quadri-velocidade de um observador comóvel é $u^{\mu}=(1,0,0,0)$.

\subsection{Dinâmica Cosmológica e Equações de Friedmann}

Para estudarmos a dinâmica cosmológica, utilizamos as equações de campo de Einstein

$$
G_{\mu \nu} \equiv R_{\mu \nu}-\frac{1}{2} g_{\mu \nu} \mathcal{R}=8 \pi G T_{\mu \nu}+\Lambda g_{\mu \nu}
$$

em que $G_{\mu \nu}$ é o tensor de Einstein, $R_{\mu \nu}$ é o tensor de Ricci, $g_{\mu \nu}$ é a métrica, $\mathcal{R}$ é o escalar de curvatura de Ricci, $G$ é a constante gravitacional, $T_{\mu \nu}$ é o tensor de energiamomento, que contém a informação sobre o conteúdo material do Universo, e $\Lambda$ é a constante cosmológica. Essa constante foi adicionada por Einstein em sua equação de campo pois, à época, acreditava-se que o Universo era estático. Com as observações de Hubble, a constante foi descartada. Como veremos mais tarde, recentes descobertas indicam que o Universo encontra-se em expansão acelerada e o candidato mais simples para explicar esse fato seria a reintrodução da constante cosmológica. Para mais detalhes sobre as equações de campo de Einstein e os passos a seguir, recomendamos a leitura dos seguintes livros-texto: [19, 20, 21, 22, 23, 24].

O tensor de Ricci é a contração do primeiro e terceiro índices do tensor de Riemann, $R_{\mu \nu}=R_{\mu \gamma \nu}^{\gamma}$. O tensor de Riemann, por sua vez, é obtido através das conexões

$$
R_{\mu \nu \eta}^{\rho}=\partial_{\nu} \Gamma_{\mu \eta}^{\rho}-\partial_{\eta} \Gamma_{\mu \nu}^{\rho}+\Gamma_{\mu \eta}^{\alpha} \Gamma_{\alpha \nu}^{\rho}-\Gamma_{\mu \nu}^{\alpha} \Gamma_{\alpha \eta}^{\rho}
$$

Assim, o tensor de Ricci fica

$$
R_{\mu \nu}=\partial_{\alpha} \Gamma_{\mu \nu}^{\alpha}-\partial_{\nu} \Gamma_{\mu \alpha}^{\alpha}+\Gamma_{\beta \alpha}^{\alpha} \Gamma_{\mu \nu}^{\beta}-\Gamma_{\beta \nu}^{\alpha} \Gamma_{\mu \alpha}^{\beta}
$$

O escalar de Ricci é o traço do tensor de Ricci

$$
\mathcal{R}=R^{\mu}{ }_{\nu}=g^{\mu \nu} R_{\mu \nu} .
$$


Passaremos agora a calcular as grandezas $\Gamma_{\mu \nu}^{\alpha}, R_{\mu \nu}$ e $\mathcal{R}$ na métrica FLRW. Para o cálculo das conexões, definimos a lagrangiana de uma partícula livre

$$
\mathcal{L}=g_{\mu \nu} \frac{d x^{\mu}}{d \lambda} \frac{d x^{\nu}}{d \lambda}
$$

em que $\lambda$ é um parâmetro arbitrário. Utilizando a métrica de FLRW (1.4), a lagrangiana pode ser escrita como

$$
\mathcal{L}=t^{\prime 2}-\frac{a^{2}(t)}{1-\kappa r^{2}} r^{\prime 2}-a^{2}(t) r^{2} \theta^{\prime 2}-a^{2}(t) r^{2} \sin ^{2} \theta \phi^{\prime 2}
$$

em que a linha se refere à derivada com relação ao parâmetro $\lambda$. Com a equação de Euler-Lagrange,

$$
\frac{d}{d \lambda} \frac{\partial \mathcal{L}}{\partial x^{\prime \alpha}}-\frac{\partial \mathcal{L}}{\partial x^{\alpha}}=0
$$

podemos encontrar as seguintes equações de movimento

$$
\begin{aligned}
t^{\prime \prime}+\frac{a}{1-\kappa r^{2}} \dot{a} r^{2}+a \dot{a} \theta^{\prime 2}+a \dot{a} r^{2} \sin ^{2} \theta \phi^{\prime 2} & =0, \\
r^{\prime \prime}+\frac{2}{a} \dot{a} r^{\prime} t^{\prime}+\frac{\kappa r}{1-\kappa r^{2}}-r\left(1-\kappa r^{2}\right) \theta^{\prime 2}-r\left(1-\kappa r^{2}\right) \sin ^{2} \theta \phi^{\prime 2} & =0, \\
\theta^{\prime \prime}+\frac{2}{a} \dot{a} \theta^{\prime} t^{\prime}+\frac{2}{r} \theta^{\prime} r^{\prime}-\sin \theta \cos \theta \phi^{\prime 2} & =0, \\
\phi^{\prime \prime}+\frac{2}{a} \dot{a} \phi^{\prime} t^{\prime}+\frac{2}{r} r^{\prime} \phi^{\prime}+2 \cot \theta \dot{\theta} \phi^{\prime} & =0 .
\end{aligned}
$$

O ponto refere-se à derivada com relação ao tempo. Comparando a equação de geodésica (1.2), que é a equação de movimento de uma partícula livre em determinada métrica, com as equações de movimento obtidas acima, encontramos as conexões não nulas, ver Tabela 1.1. 


$$
\begin{array}{|ll}
\Gamma_{11}^{0}=\frac{a \dot{a}}{1-\kappa r^{2}} & \Gamma_{02}^{2}=\frac{\dot{a}}{a} \\
\Gamma_{22}^{0}=a \dot{a} r^{2} & \Gamma_{12}^{2}=\frac{1}{r} \\
\Gamma_{33}^{0}=a \dot{a} r^{2} \sin ^{2} \theta & \Gamma_{33}^{2}=-\sin \theta \cos \theta \\
\Gamma_{01}^{1}=\frac{\dot{a}}{a} & \Gamma_{03}^{3}=\frac{\dot{a}}{a} \\
\Gamma_{11}^{1}=\frac{\kappa r}{1-\kappa r^{2}} & \Gamma_{13}^{3}=\frac{1}{r} \\
\Gamma_{22}^{1}=-r\left(1-\kappa r^{2}\right) & \Gamma_{23}^{3}=\cot \theta \\
& \Gamma_{33}^{1}=-r\left(1-\kappa r^{2}\right) \sin ^{2} \theta
\end{array}
$$

Tabela 1.1: Conexões da métrica FLRW (1.4)

De posse das conexões, podemos calcular os componentes do tensor de Ricci não nulos e o escalar de Ricci:

$$
\begin{aligned}
R_{00} & =-3 \frac{\ddot{a}}{a}, \\
R_{11} & =\frac{a \ddot{a}+2 \dot{a}^{2}+2 \kappa}{1-\kappa r^{2}}, \\
R_{22} & =r^{2}\left(a \ddot{a}+2 \dot{a}^{2}+2 k\right), \\
R_{33} & =r^{2}\left(a \ddot{a}+2 \dot{a}^{2}+2 k\right) \sin ^{2} \theta, \\
\mathcal{R} & =-6\left[\frac{\ddot{a}}{a}+\left(\frac{\dot{a}}{a}\right)^{2}+\frac{\kappa}{a^{2}}\right] .
\end{aligned}
$$

Os componentes geométricos que fazem parte das equações de Einstein foram determinados para a métrica FLRW. Ainda nos falta encontrar os componentes do tensor energia-momento. Como estamos aplicando as equações de campo para o Universo como um todo, assumiremos que o Universo é preenchido, em larga escala, por um fluido perfeito.

Ao aplicarmos às equações de Einstein (1.29) o tensor (1.28), obtemos as equações de Friedmann

$$
\begin{gathered}
\frac{\dot{a}^{2}+\kappa}{a^{2}}=\frac{8 \pi G}{3} \rho+\frac{\Lambda}{3}, \\
\frac{2 \ddot{a}}{a}+\frac{\dot{a}^{2}+\kappa}{a^{2}}=-8 \pi G p+\Lambda .
\end{gathered}
$$


Podemos juntar as duas equações de Friedmann para obter uma equação que descreve a aceleração do fator de escala:

$$
\frac{\ddot{a}}{a}=\frac{\Lambda}{3}-\frac{4 \pi G}{3}(\rho+3 p)
$$

Após as observações de Hubble, abandonou-se a constante cosmológica. Como em larga escala a força gravitacional é a força predominante e é sempre atrativa, acreditavase que a expansão observada por Hubble deveria ser desacelerada. Da equação acima, vemos que para que essa propriedade seja satisfeita basta que o conteúdo material do Universo tenha a propriedade $\rho+3 p>0$. Matéria relativística, como a radiação, possui $p=\frac{\rho}{3}$. Já a matéria não-relativística tem, nesse contexto, pressão desprezível. Como $\rho$ é sempre positivo, um Universo preenchido por matéria e radiação será sempre desacelerado. Em seções posteriores, discutiremos que a atual compreensão do Universo é a de que a aceleração é positiva. Para que isso ocorra, pode-se reintroduzir a constante cosmológica de tal forma que ela seja suficientemente positiva ou que o Universo esteja permeado por um fluido que tenha uma pressão $p<-\frac{\rho}{3}$.

Pode-se mostrar que o tensor de Einstein obedece às identidades de Bianchi

$$
\nabla_{\nu} G^{\mu \nu}=0
$$

Das equações de Einstein (1.29), vemos que o tensor de energia-momento é (supondo $\Lambda$ constante) identicamente conservado, $\nabla_{\nu} T^{\mu \nu}=0$. A componente $\mu=0$ é

$$
\nabla_{\nu} T^{\nu 0}=\frac{\partial \rho}{\partial t}+\Gamma_{0 \nu}^{\nu} T^{00}+\Gamma_{11}^{0} T^{11}+\Gamma_{22}^{0} T^{22}+\Gamma_{33}^{0} T^{33}
$$

Usando as conexões encontradas na Tabela 1.1, ficamos com

$$
\dot{\rho}+3 \frac{\dot{a}}{a}(\rho+p)=0
$$

A equação acima não é independente das equações de Friedmann. Pode-se mostrar que de (1.45) e (1.46) se obtém (1.50). Para que possamos determinar $a(t), \rho(t), p(t)$, precisamos de outra equação. Essa equação pode ser uma equação de estado conectando a pressão e a densidade, ou seja, do tipo barotrópica, $p=p(\rho)$. 


\subsection{Cosmologias do tipo FLRW}

Em geral, é difícil obter a forma exata de $a(t)$. Podemos expandir $a(t)$ em séries de Taylor em torno do tempo atual e, a partir disso, definir alguns parâmetros cosmológicos. A expansão, até termos de segunda ordem, é

$$
a(t) \approx a\left(t_{0}\right)+\left.\frac{d a}{d t}\right|_{t=t_{0}}\left(t-t_{0}\right)+\left.\frac{1}{2 !} \frac{d^{2} a}{d t^{2}}\right|_{t=t_{0}}\left(t-t_{0}\right)^{2}
$$

em que $t_{0}$ corresponde ao tempo atual. Dividindo essa equação pelo fator de escala atual e usando a normalização $a\left(t_{0}\right)=1$, ficamos cm

$$
a(t) \approx 1+H_{0}\left(t-t_{0}\right)-\frac{1}{2} q_{0} H_{0}^{2}\left(t-t_{0}\right)^{2}
$$

em que definimos os parâmetros

$$
\left.H_{0} \equiv \frac{\dot{a}}{a}\right|_{t=t_{0}}
$$

que é a constante de Hubble, e

$$
q_{0} \equiv-\left.\frac{\ddot{a} a}{\dot{a}^{2}}\right|_{t=t_{0}}=-\left.\frac{\ddot{a}}{a H^{2}}\right|_{t=t_{0}}
$$

que é chamado de parâmetro de desaceleração. No caso de $\ddot{a}>0$ o parâmetro de desaceleração mede, na verdade, a aceleração do Universo.

A equação de Friedmann (1.45) escrita com a definição de parâmetro de Hubble é dada por

$$
H^{2}=\frac{8 \pi G}{3} \rho-\frac{\kappa}{3}+\frac{\Lambda}{3}
$$

Para um Universo plano e sem constante cosmológica a equação acima reduz-se a

$$
H^{2}=\frac{8 \pi G}{3} \rho
$$

Podemos, então, definir uma densidade crítica:

$$
\rho_{c} \equiv \frac{3 H^{2}}{8 \pi G}
$$

A razão entre a densidade de qualquer componente do Universo e a densidade crítica 
recebe o nome de parâmetro de densidade:

$$
\Omega_{i} \equiv \frac{\rho_{i}}{\rho_{c}}
$$

Esse parâmetro mede a contribuição relativa de cada componente. Utilizando essa definição, podemos reescrever a equação de Friedmann (1.45) da seguinte maneira:

$$
1+\frac{\kappa}{H^{2} a^{2}}=\frac{8 \pi G}{3 H^{2}} \rho+\frac{\Lambda}{3 H^{2}}=\frac{\rho}{\rho_{c}}+\frac{\rho_{\Lambda}}{\rho_{c}},
$$

em que definimos a densidade de energia associada à constante cosmológica, $\rho_{\Lambda}=\frac{\Lambda}{8 \pi G}$. Assim, a equação acima fica

$$
\frac{\kappa}{a^{2} H^{2}}=\Omega_{\text {total }}-1
$$

em que podemos definir o parâmetro de curvatura $\Omega_{\kappa}=-\frac{\kappa}{a^{2} H^{2}}$ e $\Omega_{\text {total }}=\Sigma_{i} \Omega_{i}+\Omega_{\Lambda}$ é o parâmetro de densidade total do Universo. Dessa última expressão, vemos que se soubermos os parâmetros de densidade das diferentes componentes do Universo, podemos determinar sua curvatura. Se a densidade total de energia do Universo for maior que a densidade crítica, a soma dos parâmetros de densidade, $\Omega_{\text {total }}$, será maior que 1 e, portanto, o Universo terá curvatura positiva. Por outro lado, se a contribuição das densidades de energia for menor que a densidade crítica, $\Omega_{\text {total }}$ será menor que 1 , logo, um Universo com curvatura negativa. A última possibilidade é a densidade total de energia do Universo ser igual à densidade crítica, nesse caso teremos um Universo plano. As evidências observacionais indicam que o Universo é praticamente plano, tal que $\Omega_{\text {total }} \approx 1$.

Para estudarmos alguns modelos cosmológicos simples, utilizaremos uma equação de estado dada pela relação linear

$$
p=\omega \rho
$$

em que $\omega$ é um parâmetro que indica o tipo de fluido que estamos estudando. Alguns exemplos que trataremos aqui são: $\omega=0$ para matéria comum, $\omega=\frac{1}{3}$ para radiação e $\omega=-1$ para o vácuo.

Com a equação de estado (1.61), obtemos, através da equação de conservação de energia (1.50),

$$
\rho=\rho_{0} a^{-3(1+\omega)}
$$

Assim, a densidade da matéria é $\rho_{m}=\rho_{m, 0} a^{-3}$, em que $\rho_{m, 0}$ é a densidade de matéria 
hoje. A densidade da radiação é $\rho_{r}=\rho_{r, 0} a^{-4}$, em que $\rho_{4,0}$ é a densidade da radiação hoje. Para o vácuo, temos que a densidade seria constante. Por essa razão, é natural atribuir ao vácuo o papel de constante cosmológica.

Como o comportamento das densidades de matéria, radiação e constante cosmológica são diferentes, podemos dividir o Universo em eras distintas. Quando $a \rightarrow 0$, a densidade da radiação cresce mais rapidamente que a densidade da matéria. Então, dizemos que o Universo está na era da radiação. Com o passar do tempo e evolução de $a(t)$, a densidade da radiação cai mais rápido que a densidade da matéria. Assim, em determinado momento, a matéria passa a dominar e começa a era da matéria. Como a densidade da constante cosmológica se mantém inalterada em relação à evolução do fator de escala, em um segundo instante, quando as densidades da radiação e matéria diminuem a tal ponto que passam a ser menores que a densidade da constante cosmológica, ela, a constante cosmológica, passa a ser a componente dominante. A era da constante cosmológica é a era que vivemos atualmente. A Figura 1.4 ilustra melhor a situação. Nela, observamos a evolução das densidades da matéria, radiação e constante cosmológica.

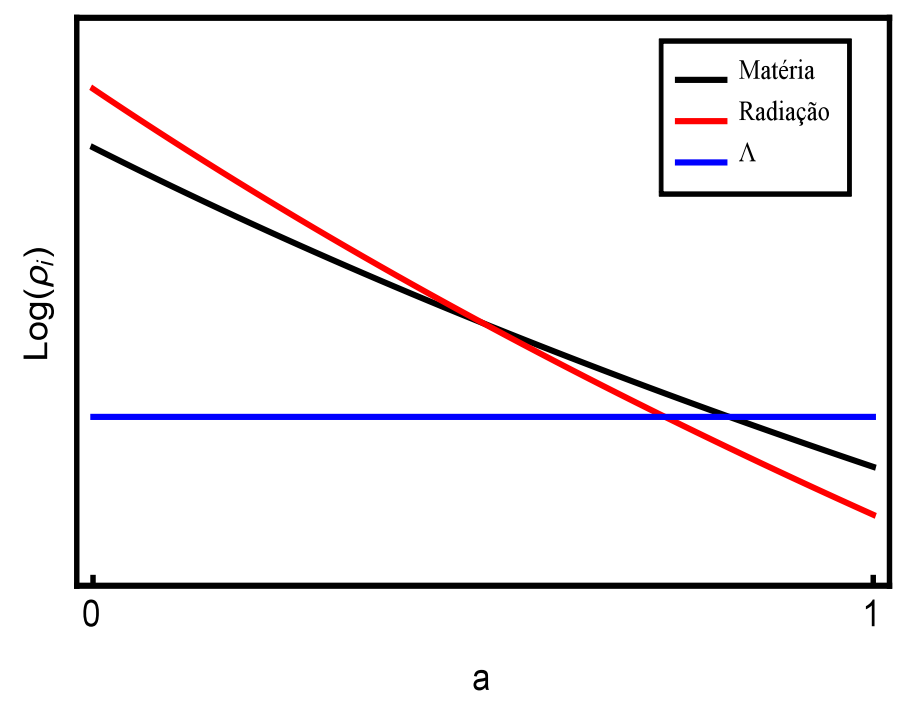

Figura 1.4: Densidades de energia de diferentes componentes em função do fator de escala $a$.

Levando em conta as definições dos parâmetros de densidade, podemos reescrever a equação de Friedmann da seguinte maneira

$$
\left(\frac{H}{H_{0}}\right)^{2}=\frac{\Omega_{r, 0}}{a^{4}}+\frac{\Omega_{m, 0}}{a^{3}}+\frac{\Omega_{\kappa, 0}}{a^{2}}+\Omega_{\Lambda, 0},
$$

em que definimos os parâmetros de densidade hoje em termos de $\rho_{c, 0}$, a densidade crítica 
hoje. $\Omega_{\kappa, 0}=1-\Omega_{\text {total }, 0}$ é o parâmetro de densidade relacionado à curvatura. Usando esse vínculo, temos a forma final

$$
H^{2}=H_{0}^{2}\left[\frac{\Omega_{r, 0}}{a^{4}}+\frac{\Omega_{m, 0}}{a^{3}}+\frac{1-\Omega_{\mathrm{total}, 0}}{a^{2}}+\Omega_{\Lambda, 0}\right]
$$

Essa é a equação de Friedmann para o modelo cosmológico $\Lambda$ CDM ( $\Lambda$ Cold Dark Matter).

Como $H \equiv \frac{\dot{a}}{a}$, podemos escrever a equação acima na forma integral

$$
H_{0} t=\int_{0}^{a} \frac{d a^{\prime}}{\left[\frac{\Omega_{r, 0}}{a^{\prime 2}}+\frac{\Omega_{m, 0}}{a^{\prime}}+1-\Omega_{\mathrm{total}, 0}+\Omega_{\Lambda, 0} a^{\prime 2}\right]^{\frac{1}{2}}} .
$$

Podemos agora fazer uma análise de alguns modelos cosmológicos simples utilizando a expressão acima para um Universo plano, $\kappa=0$.

\section{- Universo com radiação}

Em um Universo só com radiação $\left(\Omega_{r}=\Omega_{\text {total }}=1, \Omega_{m}=\Omega_{\Lambda}=0\right)$, temos

$$
H_{0} t=\int_{0}^{a} a^{\prime} d a^{\prime}
$$

cuja solução é

$$
a(t)=\left(2 H_{0} t\right)^{1 / 2} .
$$

A idade total de um Universo composto apenas por radiação é dada quando $a\left(t_{0}\right)=1$

$$
t_{0}=\frac{1}{H_{0}}
$$

\section{- Universo com matéria}

Temos agora que $\Omega_{m}=\Omega_{\text {total }}=1$ e $\Omega_{r}=\Omega_{\Lambda}=0$. Portanto, temos

$$
H_{0} t=\int_{0}^{a} a^{1 / 2} d a^{\prime}
$$

cuja solução é

$$
a(t)=\left(\frac{3}{2} H_{0} t\right)^{2 / 3} .
$$


De forma que a idade total do Universo contendo apenas matéria é

$$
t_{0}=\frac{2}{3 H_{0}}
$$

Esse modelo é conhecido como modelo de Einstein-de Sitter.

\section{- Universo com $\Lambda$}

Em um Universo dominado pela constante cosmológica temos que $\Omega_{\Lambda}=\Omega_{\text {total }}=1$ e $\Omega_{r}=\Omega_{m}=0$. Portanto, temos

$$
H_{0} t=\int_{0}^{a} \frac{d a^{\prime}}{a^{\prime}},
$$

cuja solução é

$$
a(t) \propto e^{H_{0}\left(t-t_{0}\right)}
$$

Esse modelo é conhecido como modelo de de Sitter.

A Figura 1.5 mostra a evolução do fator de escala para os três modelos estudados acima.

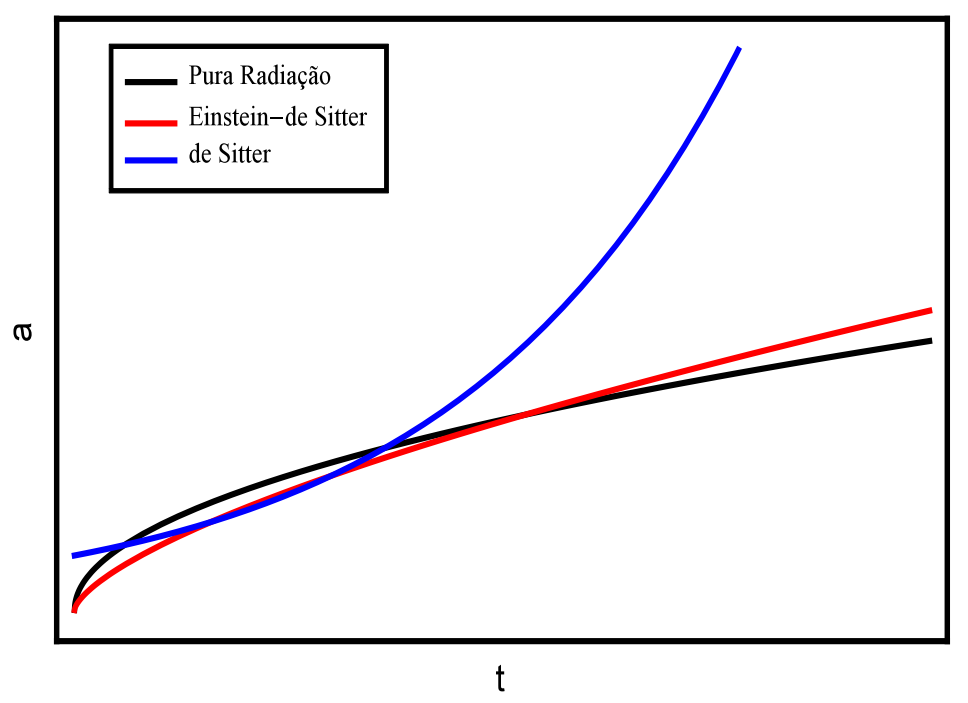

Figura 1.5: Fatores de escala para diferentes modelos cosmológicos.

Durante sua evolução, o Universo passou por fases em que cada componente (matéria, radiação etc) foi dominante. A mudança de fase, ou seja, o momento em que uma componente passou a dominar as demais, acontece quando a densidade do componente dominado passa a ser igual ao componente dominador. Como exemplo, calcularemos o instante em que a matéria passou a dominar a radiação. 
Da Figura 1.4 vemos que para instantes iniciais a radiação possui maior densidade, é a era da radiação. Com o passar do tempo, o fator de escala aumenta até certo instante em que a densidade da radiação se iguala à densidade da matéria. A partir daí, a densidade da matéria passa a ser maior que a densidade da radiação e entramos na era da matéria. Para calcularmos a idade que o Universo tinha quando aconteceu essa transição, primeiro calculamos o valor do fator de escala no momento de igualdade $a_{r m}$. Para isso, temos que $\rho_{r}\left(a_{r m}\right)=\rho_{m}\left(a_{r m}\right)$, ou

$$
a_{r m}=\frac{\rho_{r, 0}}{\rho_{m, 0}}=\frac{\Omega_{r, 0}}{\Omega_{m, 0}}=3,1 \times 10^{-4},
$$

em que usamos $\Omega_{r, 0}=9,2 \times 10^{-5}$ e $\Omega_{m, 0}=0,3$ [25]. Esses valores serão discutidos em seções posteriores.

Usando apenas matéria e radiação na integral (1.65), temos

$$
t_{r m}=H_{0}^{-1} \int_{0}^{a_{r m}} \frac{a d a}{\left[\Omega_{r, 0}\left(1+\frac{a}{a_{r m}}\right)\right]^{\frac{1}{2}}}
$$

Resolvendo a integral com $H_{0}=67.31 \mathrm{~km} / \mathrm{s} / \mathrm{Mpc}[25]$, temos

$$
t_{r m} \approx 55.661 \text { anos. }
$$

De forma similar, calculamos a idade que o Universo tinha quando a constante cosmológica passou a dominar. Igualamos as densidades de energia da matéria com a de $\Lambda$, ficando com

$$
a_{m \Lambda}=\left(\frac{\Omega_{m, 0}}{\Omega_{\Lambda, 0}}\right)^{1 / 3},
$$

que substituída na integral (1.65) e com $\Omega_{\Lambda, 0}=0.7$, fica

$$
t_{m \Lambda}=H_{0}^{-1} \int_{a_{r} m}^{a_{m \Lambda}} \frac{d a}{\left(\frac{0.3}{a}+0.7 a^{2}\right)^{1 / 2}}
$$

fornecendo uma idade de

$$
t_{m \Lambda} \approx 10.2 \times 10^{9} \text { anos }
$$

Podemos calcular a idade que um Universo plano teria se contivesse apenas matéria. 
A integral (1.65) ficaria

$$
t_{0}=H_{0}^{-1} \int_{0}^{1} a^{1 / 2} d a \approx 9.7 \times 10^{9} \text { anos }
$$

No entanto, sabe-se que a idade de alguns aglomerados de galáxias são da ordem de $12.7 \times$ $10^{9}$ anos [26]. Portanto, um Universo plano apenas com matéria estaria descartado por esse teste cosmológico. Por outro lado, um Universo com matéria e constante cosmológica teria a idade

$$
t_{0}=H_{0}^{-1} \int_{0}^{1} \frac{d a}{\left(\frac{1-\Omega_{\Lambda, 0}}{a}+\Omega_{\Lambda, 0} a^{2}\right)^{1 / 2}} .
$$

A Figura 1.6 mostra solução da integral acima para diferentes valores de $\Omega_{\Lambda, 0}$. A curva em preto mostra como a presença da constante cosmológica aumenta a idade do Universo (em anos). As curvas em vermelho e azul mostram, respectivamente, a idade dos aglomerados globulares mais antigos e a idade que o Universo teria apenas com matéria. Como se pode ver pela figura, um parâmetro de densidade da constante cosmológica da ordem $\Omega_{\Lambda, 0} \gtrsim 0.6$ aliviaria o problema da idade. Se os parâmetros de densidade da matéria e

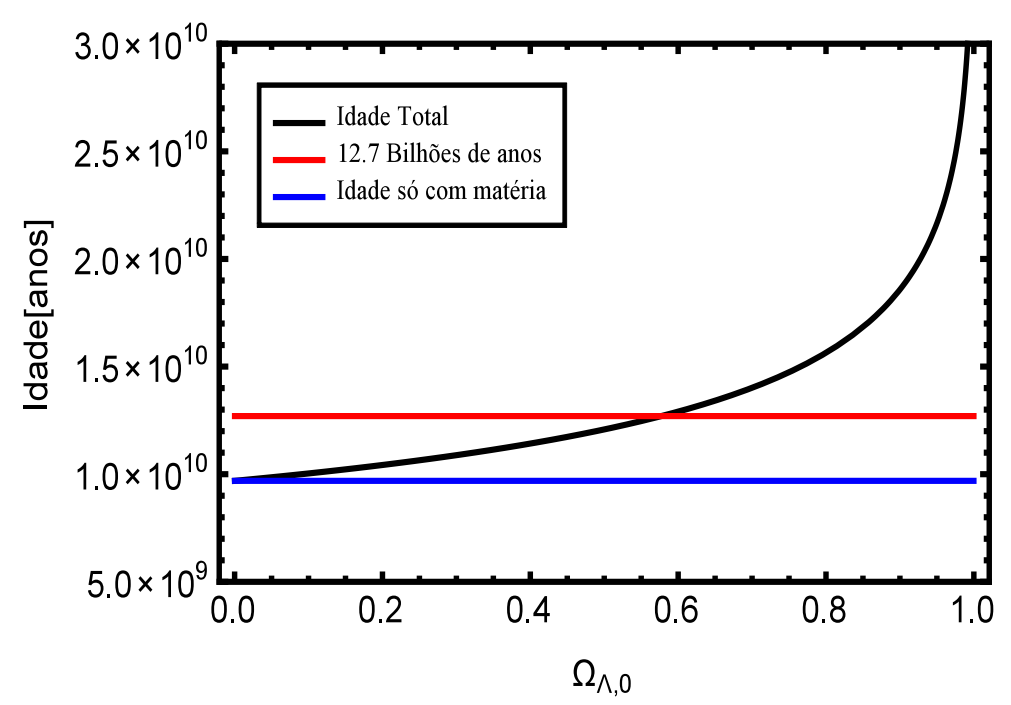

Figura 1.6: Influência de $\Lambda$ na idade total do Universo.

da constante cosmológica forem, respectivamente, $\Omega_{m, 0}=0.3$ e $\Omega_{\Lambda, 0}=0.7$, teremos uma idade total para o Universo de $\approx 14 \times 10^{9}$ anos.

O parâmetro de desaceleração para o modelo com matéria e constante cosmológica em função do redshift é

$$
q(z)=\frac{1}{2}\left(\frac{(1+z)^{3} \Omega_{m, 0}-2 \Omega_{\Lambda, 0}}{(1+z)^{3} \Omega_{m, 0}+\Omega_{\Lambda, 0}}\right) .
$$


No caso de um Universo apenas com matéria, $\Omega_{\Lambda, 0}=0$, temos que o parâmetro de desaceleração é $q=1 / 2$. Por outro lado, a presença de uma constante cosmológica faz com que esse parâmetro varie ao longo do tempo. Como podemos ver na Figura 1.7, existe um momento em que o Universo passa de uma expansão desacelerada, $q>0$, para uma expansão acelerada, $q<0$. O redshift de transição pode ser calculado impondo $q\left(z_{t}\right)=0$. Dessa forma, temos que

$$
z_{t}=\left[2 \frac{\left(1-\Omega_{m, 0}\right)}{\Omega_{m, 0}}\right]^{1 / 3}-1 .
$$

Tomando $\Omega_{m, 0}=0.287$ e $\Omega_{\Lambda, 0}=0.713$, temos $z_{t}=0.71$.

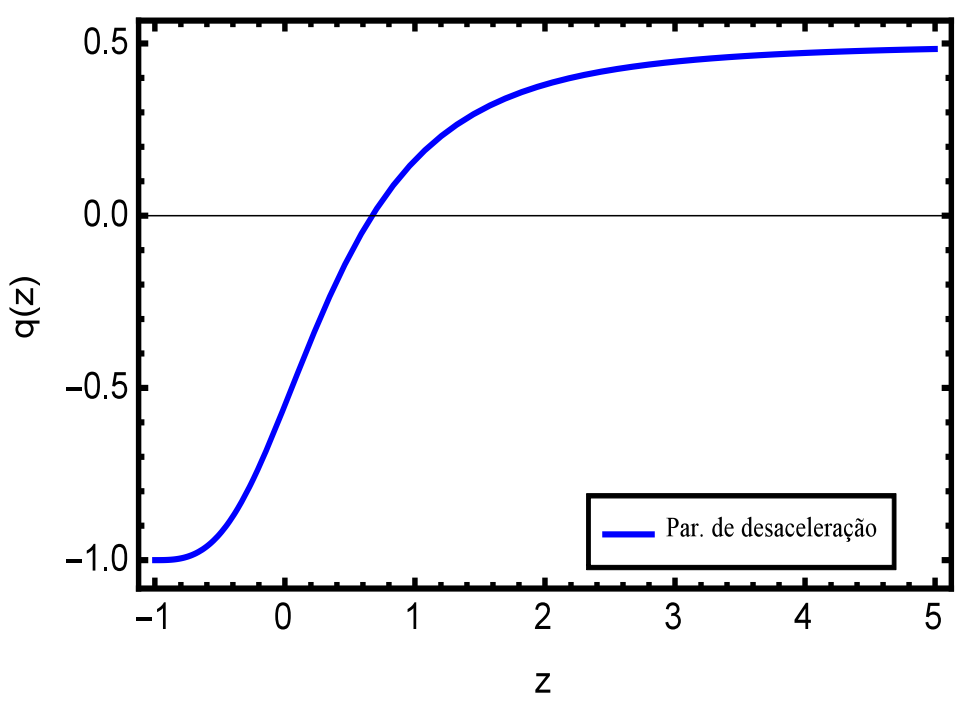

Figura 1.7: Parâmetro de desaceleração para o modelo $\Lambda$ CDM em função do redshift. 


\subsection{Distância de Luminosidade e Aceleração do Uni- verso}

Se soubermos a luminosidade $L$ de objetos a distâncias cosmológicas, podemos calcular sua distância até nós medindo o fluxo $f$ dessa fonte. Para isso, utilizamos a relação entre o fluxo observado e a luminosidade característica desse objeto com coordenada $r_{1}$ e a um certo redshift $z$

$$
f=\frac{L}{4 \pi r_{1}^{2} a^{2}\left(t_{0}\right)(1+z)^{2}} .
$$

Definimos, então, a distância de luminosidade de um objeto cosmológico a um redshift z

$$
d_{L}=a\left(t_{0}\right) r_{1}(1+z)
$$

Podemos calcular essa distância expressando a coordenada radial $r(z)$ em termos de parâmetros cosmológicos. Para isso, integramos a expressão (1.9) e utilizamos a equação de Friedmann para o modelo $\Lambda$ CDM (1.64)

$$
r(z)=S\left[\frac{1}{a_{0} H_{0}} \int_{1 /(1+z)}^{1} \frac{d x}{x^{2} \sqrt{\Omega_{\Lambda, 0}+\Omega_{\kappa} x^{-2}+\Omega_{m, 0} x^{-3}+\Omega_{r, 0} x^{-4}}}\right],
$$

em que usamos a definição $x \equiv a / a_{0}=1 /(1+z)$ e o funcional $S[y]$ é definido como

$$
S[y] \equiv\left\{\begin{array}{ll}
\sin r & \text { para } \kappa=+1 \\
r & \text { para } \kappa=0 \\
\sinh r & \text { para } \kappa=-1
\end{array} .\right.
$$

Podemos escrever a distância de luminosidade através de uma só expressão. Para isso, usamos a definição $\Omega_{\kappa}=\frac{-\kappa}{a_{0}^{2} H_{0}^{2}}$, ficando com

$$
\begin{aligned}
d_{L}(z) & =a_{0} r(z)(1+z) \\
& =\frac{1+z}{H_{0} \Omega_{\kappa}^{1 / 2}} \times \sinh \left[\Omega_{\kappa}^{1 / 2} \int_{1 /(1+z)}^{1} \frac{d x}{x^{2} \sqrt{\Omega_{\Lambda, 0}+\Omega_{\kappa} x^{-2}+\Omega_{m, 0} x^{-3}+\Omega_{r, 0} x^{-4}}}\right] .
\end{aligned}
$$

Na Figura (1.8), mostramos como a distância de luminosidade varia com o redshift para diferentes valores dos parâmetros $\left(\Omega_{\Lambda, 0}, \Omega_{m, 0}\right)$, em que consideramos um Universo plano e com contribuição da radiação insignificante. 


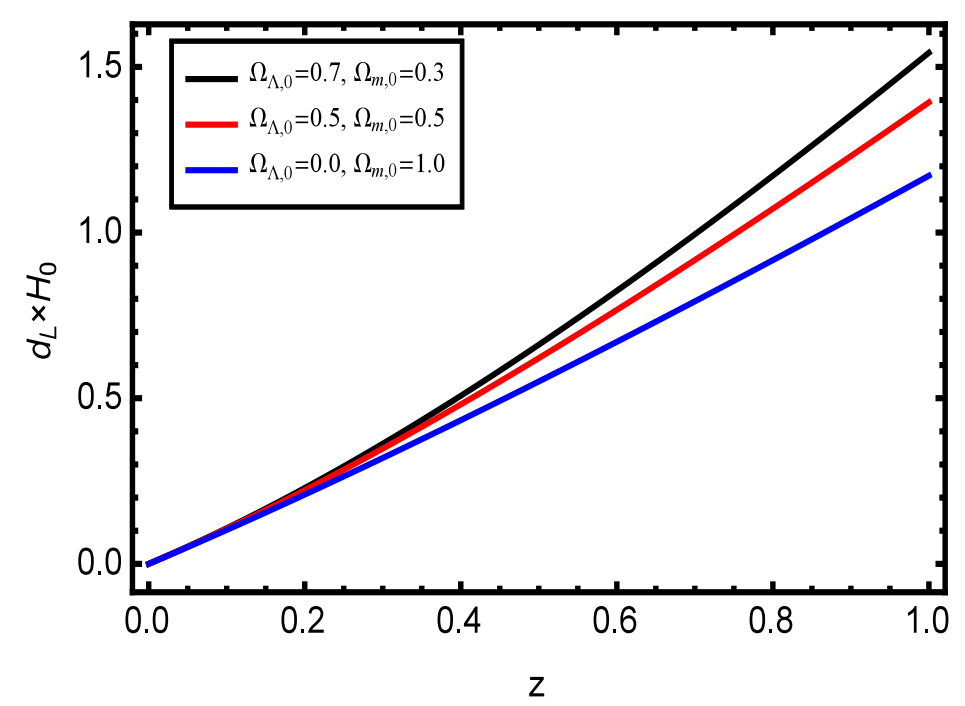

Figura 1.8: Distância de luminosidade para diferentes parâmetros.

Utilizando supernovas do tipo Ia, no fim do século passado, dois grupos, The Supernova Cosmology Project [9] e High-z Supernova Search Team [10], compararam a predição teórica da equação deduzida acima com as observações. O principal resultado dos dois grupos é que o parâmetro de densidade da matéria é $\Omega_{m, 0} \approx 0.3$ e que $\Omega_{\Lambda, 0}>0$, com $99 \%$ de nível de confiança. Esses resultados indicam um Universo em expansão acelerada. Na Figura 1.9 é mostrado um dos resultados mais recentes da compilação Union2.1, que possui dados de 580 Supernovas [2].

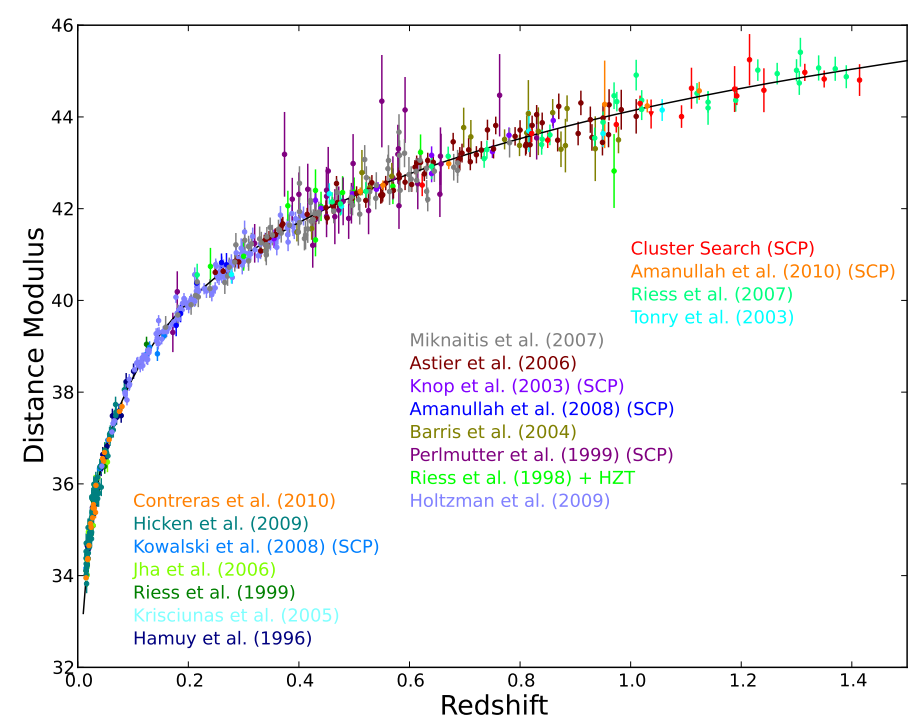

Figura 1.9: Evidência da expansão acelerada do Universo. Figura retirada de [2].

O best-fit para o modelo $\Lambda$ CDM plano do gráfico acima é $\Omega_{\Lambda, 0}=0.729 \pm 0.014$ com $68 \%$ de nível de confiança. O módulo de distância $(m-M)$, eixo das ordenadas acima, 
está relacionado com a distância de luminosidade por

$$
m-M=5 \log _{10}\left(\frac{d_{L}}{1 \mathrm{Mpc}}\right)+25,
$$

em que $m$ e $M$ são, respectivamente, a magnitude aparente e absoluta de um objeto.

A expansão acelerada do Universo é um fato inesperado. É usual associar à expansão uma nova componente do Universo, a energia escura. Ainda não sabemos a natureza da energia escura. Contudo, existem vários candidatos capazes de acelerar o Universo. Dentre eles, citamos alguns:

1. A constante cosmológica $\Lambda$ [27],

2. Um campo escalar primordial $\Phi(t)$, evoluindo em direção ao mínimo de seu potencial $[28]$,

3. Um termo $\Lambda(t)$, representando o decaimento do vácuo $[29,30,31,32,33,34,35,36]$,

4. A chamada matéria-X ou modelo XCDM [37, 38, 39],

5. O modelo do gás de Chaplygin [40, 41, 42, 43].

Ao incluirmos a matéria escura, obtemos o chamado Modelo Padrão da Cosmologia. O Universo se encontra em expansão acelerada, cuja a componente responsável pela aceleração possui $\Omega_{\Lambda} \approx 0.7$, a matéria escura contribui com $\Omega_{d m} \approx 0.26$ e podemos dizer que conhecemos a natureza do conteúdo material e energético de apenas $4 \%$ do Universo, a matéria bariônica.

Assim como a energia escura, a matéria escura possui alguns candidatos para explicar sua natureza [44]. Em capítulos posteriores, trataremos a matéria escura como WIMPs (Weakly Interacting Massive Particle), ou partícula massiva que interage fracamente. Calcularemos sua abundância hoje para o modelo $\Lambda$ CDM e para alguns modelos de criação de matéria escura. 


\section{Capítulo 2}

\section{Teoria Cinética Relativística, Equilíbrio na Métrica FLRW e}

\section{Abundâncias Cósmicas}

A teoria cinética relativística é a generalização da teoria cinética sob o prisma da teoria da relatividade. Os primeiros trabalhos nesse sentido foram propostos por Jüttner, em 1911 e 1928. No primeiro trabalho [45], ele generalizou a função de distribuição de Maxwell para o domínio da relatividade. Anos mais tarde [46], ele obteve a forma relativística para as funções de distribuição para bósons e férmions. Em 1935, Walker obteve a primeira formulação da equação de Boltzmann relativística sem colisões [47]. A versão completa da equação de Boltzmann relativística, contendo o termo de colisões, foi dada por Lichnerowicz e Marrot em 1940 [48]. A generalização do Teorema H de Boltzmann para a teoria da relatividade, que garante uma variação positiva da entropia em sistemas fora de equilíbrio, foi derivada por Marrot, Ehlers, Tauber e Weinberg e, finalmente, por Chernikov [49, 50, 51, 52].

O objetivo principal da teoria cinética (relativística ou não) é obter propriedades macroscópicas de sistemas através de suas quantidades microscópicas. Para isso, é usada a função distribuição $f\left(x^{\mu}, p^{\mu}\right)=f(x, p)$. A definição da função distribuição é tal que $f\left(x^{\mu}, p^{\mu}\right) d^{3} x d^{3} p$ fornece o número de partículas em um volume $d^{3} x$ em um ponto $\mathbf{x}$ e com momento em um intervalo $d^{3} p$ em $\boldsymbol{p}$ e em um tempo $t$. Ou seja, o número total de partículas é

$$
N=\int f\left(x^{\mu}, p^{\mu}\right) d^{3} x d^{3} p
$$


Como exemplo de como quantidades macro e microscópicas estão relacionadas, apresentamos dois tensores que são obtidos da função distribuição. Os resultados obtidos nesse capítulo são baseados nas discussões presentes em [53, 54, 55].

Começaremos nossa análise no âmbito da teoria da relatividade restrita. A partir da função distribuição, define-se a densidade de partículas

$$
n(\boldsymbol{x}, t)=\int f d^{3} p
$$

em que $f \equiv f\left(x^{\mu}, p^{\mu}\right)$. Também podemos introduzir a corrente de partículas

$$
\boldsymbol{j}(\boldsymbol{x}, t)=\int \boldsymbol{u} f d^{3} p
$$

em que $\boldsymbol{u}=\frac{\boldsymbol{p}}{p_{0}}$ é a velocidade da partícula com momento $\boldsymbol{p}$. Podemos juntar as duas definições de uma forma mais compacta e escrever o tensor de quadrifluxo de partículas

$$
N^{\mu}=\int f p^{\mu} \frac{d^{3} p}{p^{0}}
$$

Na expressão acima, $p^{\mu}$ é um tensor. Pode-se mostrar que $\frac{d^{3} p}{p^{0}}$ é um escalar invariante [53]. Portanto, para manter o caráter tensorial de $N^{\mu}$, concluímos que a função distribuição é um escalar invariante.

A densidade de energia pode ser escrita como

$$
T^{00}=\int f p^{0} d^{3} p
$$

Da mesma maneira, podemos escrever o fluxo de energia

$$
T^{0 i}=\int f p^{0} u^{i} d^{3} p
$$

A densidade de momento, que é o valor médio do momento da partícula $\boldsymbol{p}$, é

$$
T^{i 0}=\int f p^{i} d^{3} p
$$

Por fim, introduzimos o fluxo de momento (ou tensor de pressão), que é o fluxo na direção 
$j$ do momento na direção $i$

$$
T^{i j}=\int f p^{i} u^{j} d^{3} p
$$

Usando $\boldsymbol{u}=\frac{\boldsymbol{p}}{p_{0}}$, podemos escrever as quantidades acima na forma de um tensor de energiamomento

$$
T^{\mu \nu}=\int f p^{\mu} p^{\nu} \frac{d^{3} p}{p_{0}}
$$

\subsection{Equação de Boltzmann Relativística}

Passaremos a determinar a equação de Boltzmann, que dita a evolução temporal da função distribuição de um fluido. As partículas que constituem esse fluido estão sujeitas a uma série de colisões entre si. Assumiremos as seguintes condições:

- Apenas colisões binárias serão consideradas,

- Hipótese do caos molecular, os momentos das partículas antes e depois das colisões não estão correlacionados,

- A função distribuição não varia muito ao longo de um tempo que é maior que o tempo da duração da colisão mas menor que o tempo entre duas colisões nem em distâncias que são da ordem de grandeza das interações.

Tomando as coordenadas $\left(x^{\mu}, p^{\mu}\right)$ parametrizadas por um parâmetro afim $\lambda$, podemos calcular a variação de $f\left(x^{\mu}, p^{\mu}\right)$ ao longo de $\lambda$. Essa variação é devida a um termo que leva em conta as colisões entre as partículas, o chamado termo colisional $\mathcal{C}[f]$

$$
\frac{d}{d \lambda} f\left(x^{\mu}(\lambda), p^{\mu}(\lambda)\right) \equiv \frac{\partial f}{\partial x^{\mu}} \frac{d x^{\mu}}{d \lambda}+\frac{\partial f}{\partial p^{\mu}} \frac{d p^{\mu}}{d \lambda}=\mathcal{C}[f]
$$

Da equação da geodésica (1.2) e da definição de quadrimomento $p^{\mu}=\frac{d x^{\mu}}{d \lambda}$, podemos reescrever

$$
\frac{d f}{d \lambda} \equiv \mathcal{L}[f] \equiv p^{\mu} \frac{\partial f}{\partial x^{\mu}}-\Gamma_{\alpha \beta}^{\mu} p^{\alpha} p^{\beta} \frac{\partial f}{\partial p^{\mu}}=\mathcal{C}[f],
$$

em que $\mathcal{L}[f]$ é conhecido como operador de Liouville. No caso da relatividade especial, os símbolos de Christoffel se anulam, fornecendo

$$
p^{\mu} \frac{\partial f}{\partial x^{\mu}}=\mathcal{C}[f]
$$


A variação da função distribuição está ligada a uma mudança no número de partículas num intervalo $\Delta^{4} x$ e $\Delta^{3} p$. Essa mudança é resultado das colisões entre partículas e podemos escrever como [54]

$$
\Delta^{4} x \frac{\Delta^{3} p}{p^{0}} \mathcal{C}(x, p)
$$

Para determinar a forma de $\mathcal{C}(x, p)$ precisamos considerar uma colisão entre duas partículas com quadrimomentos iniciais $p^{\mu}$ e $p_{1}^{\mu}$ e quadrimomentos finais $p^{\prime \mu}$ e $p_{1}^{\prime \mu}$ e assumir que o número médio das colisões em um elemento $\Delta^{4} x$ centrado em $x$ é proporcional:

1. ao número médio de partículas por unidade de volume com trimomento $(\boldsymbol{p}, \boldsymbol{p}+\Delta \boldsymbol{p})$, ou seja, $\Delta^{3} p f(x, p)$,

2. ao número médio de partículas por unidade de volume com trimomento $\left(\boldsymbol{p}_{\mathbf{1}}, \boldsymbol{p}_{\mathbf{1}}+\right.$ $\left.\Delta \boldsymbol{p}_{1}\right)$, ou seja, $\Delta^{3} p_{1} f\left(x, p_{1}\right)$, e

3. aos intervalos $\Delta^{3} p^{\prime}, \Delta^{3} p_{1}^{\prime}$ e $\Delta^{4} x$.

O fator de proporcionalidade, denotado por $\frac{W\left(p, p_{1} \mid p^{\prime}, p_{1}^{\prime}\right)}{p^{0} p_{1}^{0} p^{\prime 0} p_{1}^{\prime 0}}$, também chamado de taxa de transição, depende apenas dos quadrimomentos antes e depois da colisão e é um escalar de Lorentz.

Para estar de acordo com a condição de que a função distribuição muda devagar ao longo de distâncias e tempos da ordem dos comprimentos e tempos característicos de interação, é suposto que a diferença das coordenadas no espaço-tempo das partículas colisionais antes e depois da colisão podem ser desprezadas. A dependência nas coordenadas $x^{\mu}$ do espaço-tempo é tal que o mesmo valor figura nas funções distribuição $f(x, p)$ e $f\left(x, p_{1}\right)$, enquanto é ausente na taxa de transição $W\left(p, p_{1} \mid p^{\prime}, p_{1}^{\prime}\right)$.

Podemos dizer que o número médio de partículas, no intervalo $\Delta^{4} x$ e com momento no intervalo $(\boldsymbol{p}, \boldsymbol{p}+\Delta \boldsymbol{p})$, que são perdidas através das colisões, é obtido ao integrarmos o número descrito acima sobre todos os valores de $\boldsymbol{p}_{\mathbf{1}}, \boldsymbol{p}^{\prime}$ e $\boldsymbol{p}_{\mathbf{1}}^{\prime}$. Assim, o número de partículas que são perdidas é

$$
\Delta^{4} x \frac{\Delta^{3} p}{p^{0}} \int f(x, p) f\left(x, p_{1}\right) W\left(p, p_{1} \mid p^{\prime}, p_{1}^{\prime}\right) \frac{d^{3} p_{1}}{p_{1}^{0}} \frac{d^{3} p^{\prime}}{p^{\prime}} \frac{d^{3} p_{1}^{\prime}}{p_{1}^{\prime 0}}
$$

Da mesma maneira, se obtém o ganho de partículas no intervalo $\Delta^{4} x$ e $\Delta^{3} p$ em $x$ e $\boldsymbol{p}$ a partir das colisões restituidoras, com momentos iniciais $\left(\boldsymbol{p}^{\prime}, \boldsymbol{p}_{\mathbf{1}}^{\prime}\right)$ e momentos finais $\left(\boldsymbol{p}, \boldsymbol{p}_{\mathbf{1}}\right)$, 
com o número

$$
\Delta^{4} x \frac{\Delta^{3} p}{p^{0}} \int f\left(x, p^{\prime}\right) f\left(x, p_{1}^{\prime}\right) W\left(p^{\prime}, p_{1}^{\prime} \mid p, p_{1}\right) \frac{d^{3} p_{1}}{p_{1}^{0}} \frac{d^{3} p^{\prime}}{p^{\prime 0}} \frac{d^{3} p_{1}^{\prime}}{p_{1}^{\prime 0}}
$$

Então, o número líquido de partículas nos intervalos $\Delta^{4} x$ e $\Delta^{3} p$ é a diferença das duas integrais acima. Portanto, o termo de colisão é

$$
\mathcal{C}(x, p)=\int\left[f^{\prime} f_{1}^{\prime} W\left(p^{\prime}, p_{1}^{\prime} \mid p, p_{1}\right)-f f_{1} W\left(p, p_{1} \mid p^{\prime}, p_{1}^{\prime}\right)\right] \frac{d^{3} p_{1}}{p_{1}^{0}} \frac{d^{3} p^{\prime}}{p^{\prime 0}} \frac{d^{3} p_{1}^{\prime}}{p_{1}^{\prime 0}}
$$

em que $f=f(x, p), f_{1}=f\left(x, p_{1}\right), f^{\prime}=f\left(x, p^{\prime}\right)$ e $f_{1}^{\prime}=f\left(x, p_{1}^{\prime}\right)$.

Como discutido em [53], alguns autores adicionam um fator $1 / 2$ na frente das integrais discutidas acima. Isso vem do fato de que, se considerarmos o caso quântico, diferente do caso clássico, não conseguimos distinguir os estados finais $\left(\boldsymbol{p}^{\prime}, \boldsymbol{p}_{\mathbf{1}}^{\prime}\right)$ dos estados $\left(\boldsymbol{p}_{\mathbf{1}}^{\prime}, \boldsymbol{p}^{\prime}\right)$. Adicionaremos o fator $1 / 2$ sempre que for necessário, ou seja, se formos considerar o caso quântico em determinado momento no texto.

A taxa de transição tem a propriedade $W\left(p^{\prime}, p_{1}^{\prime} \mid p, p_{1}\right)=W\left(p, p_{1} \mid p^{\prime}, p_{1}^{\prime}\right)$. Portanto, com a construção do termo de colisão descrita acima, podemos escrever a equação de Boltzmann da seguinte forma

$$
p^{\mu} \frac{\partial f}{\partial x^{\mu}}-\Gamma_{\alpha \beta}^{\mu} p^{\alpha} p^{\beta} \frac{\partial f}{\partial p^{\mu}}=\int\left[f^{\prime} f_{1}^{\prime}-f f_{1}\right] \times W\left(p, p_{1} \mid p^{\prime}, p_{1}^{\prime}\right) \frac{d^{3} p_{1}}{p_{1}^{0}} \frac{d^{3} p^{\prime}}{p^{\prime 0}} \frac{d^{3} p_{1}^{\prime}}{p_{1}^{\prime 0}}
$$

Quando se levam em conta as estatísticas quânticas, o termo $f f_{1}$ deve ser substituído por

$$
f f_{1} \rightarrow f f_{1}\left(1+\varepsilon \frac{f^{\prime} h^{3}}{g_{s}}\right)\left(1+\varepsilon \frac{f_{1}^{\prime} h^{3}}{g_{s}}\right)
$$

em que $h$ é a constante de Planck, $g_{s}$ é o fator de degenerescência e $\varepsilon=0,-1,+1$ para estatística de Maxwell-Boltzmann, Fermi-Dirac e Bose-Einstein, respectivamente. Portanto, a equação de Boltzmann no espaço de Minkowski fica

$$
\begin{aligned}
p^{\mu} \frac{\partial f}{\partial x^{\mu}}= & \int\left[f^{\prime} f_{1}^{\prime}\left(1+\varepsilon \frac{f h^{3}}{g_{s}}\right)\left(1+\varepsilon \frac{f_{1} h^{3}}{g_{s}}\right)\right. \\
& \left.-f f_{1}\left(1+\varepsilon \frac{f^{\prime} h^{3}}{g_{s}}\right)\left(1+\varepsilon \frac{f_{1}^{\prime} h^{3}}{g_{s}}\right)\right] \times \\
& W\left(p, p_{1} \mid p^{\prime}, p_{1}^{\prime}\right) \frac{d^{3} p_{1}}{p_{1}^{0}} \frac{d^{3} p^{\prime}}{p^{\prime 0}} \frac{d^{3} p_{1}^{\prime}}{p_{1}^{0}}
\end{aligned}
$$




\subsection{Equação de Transferência e Invariantes de Soma}

No espaço de Minkowski, a equação de Boltzmann fica

$$
p^{\mu} \frac{\partial f}{\partial x^{\mu}}=\int\left[f^{\prime} f_{1}^{\prime}-f f_{1}\right] W\left(p, p_{1} \mid p^{\prime}, p_{1}^{\prime}\right) \frac{d^{3} p_{1}}{p_{1}^{0}} \frac{d^{3} p^{\prime}}{p^{\prime 0}} \frac{d^{3} p_{1}^{\prime}}{p_{1}^{\prime 0}}
$$

Multiplicando a equação acima por uma função arbitrária $\psi\left(x^{\nu}, p^{\nu}\right)$ e integrando com relação a $\frac{d^{3} p}{p^{0}}$, ficamos com

$$
\int \psi p^{\mu} \frac{\partial f}{\partial x^{\mu}} \frac{d^{3} p}{p^{0}}=\int \psi\left[f^{\prime} f_{1}^{\prime}-f f_{1}\right] W\left(p, p_{1} \mid p^{\prime}, p_{1}^{\prime}\right) \frac{d^{3} p}{p^{0}} \frac{d^{3} p_{1}}{p_{1}^{0}} \frac{d^{3} p^{\prime}}{p^{\prime 0}} \frac{d^{3} p_{1}^{\prime}}{p_{1}^{\prime 0}}
$$

O primeiro termo pode ser reescrito da seguinte maneira:

$$
\int \psi p^{\mu} \frac{\partial f}{\partial x^{\mu}} \frac{d^{3} p}{p^{0}}=\frac{\partial}{\partial x^{\mu}} \int \psi p^{\mu} f \frac{d^{3} p}{p^{0}}-\int p^{\mu} f \frac{\partial \psi}{\partial x^{\mu}} \frac{d^{3} p}{p^{0}}
$$

Passaremos agora a reescrever a parte da direita da equação (2.21). Primeiramente, denotaremos por $\tau$ a integral da direita. Tomemos o primeiro termo e troquemos os papeis das partículas antes e depois da colisão. Ou seja, $\left(p^{\prime \mu}, p_{1}^{\prime \mu}\right) \rightarrow\left(p^{\mu}, p_{1}^{\mu}\right)$. Como $W\left(p^{\prime}, p_{1}^{\prime} \mid p, p_{1}\right)=W\left(p, p_{1} \mid p^{\prime}, p_{1}^{\prime}\right)$, temos

$$
\tau=\int\left(\psi^{\prime}-\psi\right) f f_{1} W\left(p, p_{1} \mid p^{\prime}, p_{1}^{\prime}\right) \frac{d^{3} p}{p^{0}} \frac{d^{3} p_{1}}{p_{1}^{0}} \frac{d^{3} p^{\prime}}{p^{\prime 0}} \frac{d^{3} p_{1}^{\prime}}{p_{1}^{\prime 0}}
$$

Agora, trocando $\left(p^{\mu}, p^{\prime \mu}\right) \rightarrow\left(p_{1}^{\mu}, p_{1}^{\prime \mu}\right)$,

$$
\tau=\int\left(\psi_{1}^{\prime}-\psi_{1}\right) f f_{1} W\left(p, p_{1} \mid p^{\prime}, p_{1}^{\prime}\right) \frac{d^{3} p}{p^{0}} \frac{d^{3} p_{1}}{p_{1}^{0}} \frac{d^{3} p^{\prime}}{p^{\prime 0}} \frac{d^{3} p_{1}^{\prime}}{p_{1}^{0}}
$$

Se fizermos a semi-soma dos dois termos acima temos

$$
\begin{aligned}
\tau & =\frac{1}{2} \int\left(\psi^{\prime}+\psi_{1}^{\prime}-\psi-\psi_{1}\right) f f_{1} W\left(p, p_{1} \mid p^{\prime}, p_{1}^{\prime}\right) \frac{d^{3} p}{p^{0}} \frac{d^{3} p_{1}}{p_{1}^{0}} \frac{d^{3} p^{\prime}}{p^{\prime 0}} \frac{d^{3} p_{1}^{\prime}}{p_{1}^{\prime 0}} \\
& =\frac{1}{4} \int\left(\psi+\psi_{1}-\psi^{\prime}-\psi_{1}^{\prime}\right)\left(f^{\prime} f_{1}^{\prime}-f f_{1}\right) W\left(p, p_{1} \mid p^{\prime}, p_{1}^{\prime}\right) \frac{d^{3} p}{p^{0}} \frac{d^{3} p_{1}}{p_{1}^{0}} \frac{d^{3} p^{\prime}}{p^{\prime 0}} \frac{d^{3} p_{1}^{\prime}}{p_{1}^{\prime 0}}
\end{aligned}
$$

em que no último passo foi usado de novo $\left(p^{\prime \mu}, p_{1}^{\prime \mu}\right) \rightarrow\left(p^{\mu}, p_{1}^{\mu}\right)$. 
Portanto, a equação de transferência é

$$
\begin{aligned}
& \frac{\partial}{\partial x^{\mu}}\left[\int \psi p^{\mu} f \frac{d^{3} p}{p^{0}}\right]-\int p^{\mu} f \frac{\partial \psi}{\partial x^{\mu}} \frac{d^{3} p}{p^{0}} \\
& =\frac{1}{4} \int\left(\psi+\psi_{1}-\psi^{\prime}-\psi_{1}^{\prime}\right)\left(f^{\prime} f_{1}^{\prime}-f f_{1}\right) W\left(p, p_{1} \mid p^{\prime}, p_{1}^{\prime}\right) \frac{d^{3} p}{p^{0}} \frac{d^{3} p_{1}}{p_{1}^{0}} \frac{d^{3} p^{\prime}}{p^{\prime 0}} \frac{d^{3} p_{1}^{\prime}}{p_{1}^{0}}
\end{aligned}
$$

Ou ainda, se levarmos em conta as diferentes estatísticas, ficamos com

$$
\begin{aligned}
& \frac{\partial}{\partial x^{\mu}}\left[\int \psi p^{\mu} f \frac{d^{3} p}{p^{0}}\right]-\int p^{\mu} f \frac{\partial \psi}{\partial x^{\mu}} \frac{d^{3} p}{p^{0}}= \\
& \frac{1}{4} \int\left(\psi+\psi_{1}-\psi^{\prime}-\psi_{1}^{\prime}\right)\left[f^{\prime} f_{1}^{\prime}\left(1+\varepsilon \frac{f h^{3}}{g_{s}}\right)\left(1+\varepsilon \frac{f_{1} h^{3}}{g_{s}}\right)\right. \\
& \left.-f f_{1}\left(1+\varepsilon \frac{f^{\prime} h^{3}}{g_{s}}\right)\left(1+\varepsilon \frac{f_{1}^{\prime} h^{3}}{g_{s}}\right)\right] W\left(p, p_{1} \mid p^{\prime}, p_{1}^{\prime}\right) \frac{d^{3} p}{p^{0}} \frac{d^{3} p_{1}}{p_{1}^{0}} \frac{d^{3} p^{\prime}}{p^{\prime 0}} \frac{d^{3} p_{1}^{\prime}}{p_{1}^{\prime 0}}
\end{aligned}
$$

Funções do tipo

$$
\psi(x, p)=a(x)+b_{\mu}(x) p^{\mu}
$$

são ditas invariantes de soma. Devido à conservação do quadrimomento, funções desse tipo têm a propriedade de zerar o lado direito da equação (2.26).

\subsection{Entropia e Função Distribuição de Equilíbrio}

Uma escolha interessante para a função arbitrária $\psi$ é

$$
\psi=-k c\left[\ln \left(\frac{f h^{3}}{g_{s}}\right)-\left(1+\frac{g_{s}}{\varepsilon f h^{3}}\right) \ln \left(1+\frac{\varepsilon f h^{3}}{g_{s}}\right)\right]
$$

que, substituída em (2.26), produz uma equação de balanço do tipo

$$
\frac{\partial S^{\mu}}{\partial x^{\mu}}=\varsigma
$$


em que $h$ é a constante de Planck - introduzida para deixar o argumento do logaritmo adimensional - em que

$$
S^{\mu}=-k c \int p^{\mu} f\left[\ln \left(\frac{f h^{3}}{g_{s}}\right)-\left(1+\frac{g_{s}}{\varepsilon f h^{3}}\right) \ln \left(1+\frac{\varepsilon f h^{3}}{g_{s}}\right)\right] \frac{d^{3} p}{p^{0}}
$$

é o fluxo de entropia e

$$
\begin{aligned}
\varsigma= & \frac{k c}{4} \int f f_{1} \ln \left[\frac{f^{\prime} f_{1}^{\prime}\left(1+\varepsilon f h^{3} / g_{s}\right)\left(1+\varepsilon f_{1} h^{3} / g_{s}\right)}{f f_{1}\left(1+\varepsilon f^{\prime} h^{3} / g_{s}\right)\left(1+\varepsilon f_{1}^{\prime} h^{3} / g_{s}\right)}\right]\left(1+\varepsilon \frac{f^{\prime} h^{3}}{g_{s}}\right) \times \\
& \left(1+\varepsilon \frac{f_{1}^{\prime} h^{3}}{g_{s}}\right)\left[\frac{f^{\prime} f_{1}^{\prime}\left(1+\varepsilon f h^{3} / g_{s}\right)\left(1+\varepsilon f_{1} h^{3} / g_{s}\right)}{f f_{1}\left(1+\varepsilon f^{\prime} h^{3} / g_{s}\right)\left(1+\varepsilon f_{1}^{\prime} h^{3} / g_{s}\right)}-1\right] \times \\
& W\left(p, p_{1} \mid p^{\prime}, p_{1}^{\prime}\right) \frac{d^{3} p}{p^{0}} \frac{d^{3} p_{1}}{p_{1}^{0}} \frac{d^{3} p^{\prime}}{p^{\prime}} \frac{d^{3} p_{1}^{\prime}}{p_{1}^{\prime 0}}
\end{aligned}
$$

é a taxa de produção de entropia. Como $(x-1) \ln x \geq 0, \varsigma \geq 0$. Isso quer dizer que, em processos como esse, a entropia permanece constante ou aumenta (segunda lei da termodinâmica).

Para processos em equilíbrio, a produção de entropia deve ser nula, ou seja, $\varsigma=0$. Isso significa que

$$
\begin{aligned}
& f^{\prime 0} f_{1}^{\prime 0}\left(1+\varepsilon \frac{f^{0} h^{3}}{g_{s}}\right)\left(1+\varepsilon \frac{f_{1}^{0} h^{3}}{g_{s}}\right)= \\
& f^{0} f_{1}^{0}\left(1+\varepsilon \frac{f^{\prime 0} h^{3}}{g_{s}}\right)\left(1+\varepsilon \frac{f_{1}^{\prime 0} h^{3}}{g_{s}}\right),
\end{aligned}
$$

em que o 0 em sobrescrito representa quantidades de equilíbrio. É importante notar que a condição acima também zera o lado direito da equação de Boltzmann. Ou seja, o termo colisional $\mathcal{C}$ é zerado com a introdução da função distribuição de equilíbrio.

Tomando o logaritmo dos dois lados da equação acima, ficamos com

$$
\begin{aligned}
& \ln \left(\frac{f^{0}}{1+\varepsilon f^{0} h^{3} / g_{s}}\right)+\ln \left(\frac{f_{1}^{0}}{1+\varepsilon f_{1}^{0} h^{3} / g_{s}}\right)= \\
& \ln \left(\frac{f^{\prime 0}}{1+\varepsilon f^{\prime 0} h^{3} / g_{s}}\right)+\ln \left(\frac{f_{1}^{\prime 0}}{1+\varepsilon f_{1}^{\prime 0} h^{3} / g_{s}}\right) .
\end{aligned}
$$

Portanto, $\ln \left(\frac{f^{0}}{1+\varepsilon f^{0} h^{3} / g_{s}}\right)$ é um invariante de soma e deve ter a forma (2.28), que pode ser escrita como

$$
\ln \left(\frac{f^{0}}{1+\varepsilon f^{0} h^{3} / g_{s}}\right)=a^{\prime}-b^{\mu} p_{\mu}
$$


ou ainda

$$
f^{0}=\frac{g_{s} / h^{3}}{e^{-a+b^{\mu} p_{\mu}}-\varepsilon}
$$

em que $a=a(x), b^{\mu}=b^{\mu}(x)$ para economizar notação e $a=a^{\prime}-\ln g_{s} / h^{3}$. O sinal "_" é para garantir que $f^{0}$ convirja para $p \rightarrow \infty$.

Primeiro determinaremos o parâmetro $b^{\mu}$. Para isso, o escrevemos em um referencial em repouso:

$$
b_{R}^{\mu}=\left(\frac{\beta}{m c}, \mathbf{0}\right)
$$

em que o parâmetro $\beta$ será determinado mais tarde. Como $b^{\mu}$ é um quadrivetor, seu módulo é o mesmo para qualquer observador, ou seja

$$
b_{R}^{\mu} b_{R \mu}=b^{\mu} b_{\mu}=\frac{\beta^{2}}{(m c)^{2}}
$$

Da expressão acima podemos estabelecer a seguinte relação:

$$
\frac{\partial \beta}{\partial b_{\mu}}=\frac{(m c)^{2}}{\beta} b^{\mu}
$$

Ao inserirmos a função distribuição de equilíbrio na definição de fluxo de partículas, ficamos com

$$
N_{E}^{\mu}=c \int p^{\mu} \frac{g_{s} / h^{3}}{e^{-a+b^{\mu} p_{\mu}}-\varepsilon} \frac{d^{3} p}{p_{0}},
$$

que pode ser escrita da seguinte maneira:

$$
\frac{\partial N_{E}^{\mu}}{\partial a}=-c \frac{\partial \mathcal{I}}{\partial b_{\mu}}
$$

em que

$$
\mathcal{I}=\int \frac{g_{s} / h^{3}}{e^{-a+b^{\nu} p_{\nu}}-\varepsilon} \frac{d^{3} p}{p_{0}}
$$

Em um sistema de referência em repouso temos que

$$
d^{3} p=p^{2} d p d \Omega
$$

em que $d \Omega$ é o diferencial de ângulo sólido. Vamos introduzir uma nova variável tal que

$$
b_{R}^{\mu} p_{\mu}=\frac{p_{0} \beta}{m c}=\beta \cosh \vartheta, p^{2}=m^{2} c^{2} \sinh ^{2} \vartheta, \frac{d^{3} p}{p_{0}}=m^{2} c^{2} \sinh ^{2} \vartheta d \vartheta d \Omega .
$$


Definimos as integrais

$$
J_{n m}(\beta, a)=\int_{0}^{\infty} \frac{\sinh ^{n} \vartheta \cosh ^{m} \vartheta}{e^{\beta \cosh \vartheta-a}-\varepsilon} d \vartheta
$$

que possuem as propriedades

$$
\begin{gathered}
\frac{\partial J_{n m}}{\partial a}=\frac{n-1}{\beta} J_{n-2, m+1}+\frac{m}{\beta} J_{n, m-1}, \\
\frac{\partial J_{n m}}{\partial \beta}=-\frac{n-1}{\beta} J_{n-2, m+2}-\frac{m+1}{\beta} J_{n, m}=-\frac{\partial J_{n, m+1}}{\partial a} .
\end{gathered}
$$

Num sistema em repouso, a densidade de partículas é dada por

$$
n=c^{-1} N_{E}^{\mu} U_{\mu}=\frac{g_{s}}{h^{3}} \int \frac{p^{0}}{e^{\frac{\beta p_{0}}{m c}}-\varepsilon} \frac{d^{3} p}{p^{0}},
$$

ou, em termos das integrais $J$,

$$
n=4 \pi(m c)^{3} \frac{g_{s}}{h^{3}} J_{21}
$$

Da mesma maneira, a integral $\mathcal{I}$ escrita em termos das integrais $J$ fica

$$
\mathcal{I}=4 \pi(m c)^{2} \frac{g_{s}}{h^{3}} J_{20}
$$

Usando as propriedades das derivadas das integrais $J$, encontramos a seguinte relação:

$$
\frac{\partial n}{\partial a}=-\frac{1}{m c} \frac{\partial \mathcal{I}}{\partial \beta}
$$

Com isso, da definição

$$
N_{E}^{\mu}=n U^{\mu},
$$

tiramos que

$$
\frac{\partial N_{E}^{\mu}}{\partial a}=-\frac{1}{m c} \frac{\partial \mathcal{I}}{\partial \beta} U^{\mu} .
$$

Por outro lado,

$$
\frac{\partial N_{E}^{\mu}}{\partial a}=-c \frac{\partial \mathcal{I}}{\partial b_{\mu}}=-c \frac{\partial \mathcal{I}}{\partial \beta} \frac{\partial \beta}{\partial b_{\mu}}=-c \frac{(m c)^{2}}{\beta} \frac{\partial \mathcal{I}}{\partial \beta} b^{\mu}
$$


Portanto, comparando as duas expressões acima, concluímos que

$$
b^{\mu}=\frac{\beta}{m c^{2}} U^{\mu}
$$

Para determinarmos $\beta$ e $a$, escrevamos as expressões de equilíbrio em um referencial em repouso, para a densidade de energia e para a pressão, em termos das integrais $J$ :

$$
\rho=4 \pi m^{4} c^{5} \frac{g_{s}}{h^{3}} J_{22}, \quad p=\frac{4 \pi}{3} m^{4} c^{5} \frac{g_{s}}{h^{3}}\left(J_{22}-J_{20}\right)=\frac{4 \pi}{3} m^{4} c^{5} \frac{g_{s}}{h^{3}} J_{40}
$$

em que foi utilizado

$$
\rho=\frac{1}{c^{2}} T_{E}^{\mu \nu} U_{\mu} U_{\nu},-3 p+\rho=T_{E}^{\mu \nu} \eta_{\mu \nu}
$$

Calculando a entropia por partícula, $\sigma_{E}$, dada por

$$
\begin{aligned}
\sigma_{E}= & \frac{1}{n c^{2}} S_{E}^{\mu} U_{\mu} \\
= & \frac{k}{n} \int p_{0} \frac{d^{3} p}{p_{0}} f^{0}\left[\ln \left(e^{-a+\beta \cosh \vartheta}-\varepsilon\right)+\frac{e^{-a+\beta \cosh \vartheta}}{\varepsilon} \ln \left(\frac{e^{-a+\beta \cosh \vartheta}}{e^{-a+\beta \cosh \vartheta}-\varepsilon}\right)\right] \\
= & \frac{k}{n} \int p_{0} \frac{d^{3} p}{p_{0}} f^{0}(\beta \cosh \vartheta-a)+\frac{g_{s} k}{n \varepsilon h^{3}} \int p_{0} \frac{d^{3} p}{p_{0}}(\beta \cosh \vartheta-a) \\
& -\frac{g_{s} k}{n \varepsilon h^{3}} \int p_{0} \frac{d^{3} p}{p_{0}} \ln e^{-a+\beta \cosh \vartheta}-\varepsilon \\
= & \frac{4 \pi k g_{s}(m c)^{3}}{n h^{3}} \times\left\{\int \frac{\cosh \vartheta \sinh ^{2} \vartheta(\beta \cosh \vartheta-a)}{e^{-a+\beta \cosh \vartheta}-\varepsilon} d \vartheta\right. \\
& \left.\frac{1}{\varepsilon} \int \cosh \vartheta \sinh ^{2} \vartheta\left[\beta \cosh \vartheta-a-\ln \left[e^{-a+\beta \cosh \vartheta} \times\left(1-\varepsilon e^{a-\beta \cosh \vartheta}\right)\right]\right] d \vartheta\right\} \\
= & \frac{4 \pi k g_{s}(m c)^{3}}{n h^{3}} \times\left\{\left(\beta J_{22}-a J_{21}\right)\right. \\
& \left.-\frac{1}{\varepsilon} \int \cosh \vartheta \sinh ^{2} \vartheta \ln \left(1-\varepsilon e^{a-\beta \cosh \vartheta}\right) d \vartheta\right\}
\end{aligned}
$$

Com uma integração por partes na última integral, ficamos com o resultado

$$
\sigma_{E}=k\left(\frac{\beta}{m c^{2}} e-a+\frac{4 \pi m^{3} c^{3} g_{s}}{3 h^{3}} \frac{\beta}{n} J_{40}\right)
$$


Tomando o diferencial da equação acima

$$
\begin{aligned}
d \sigma_{E}= & k\left\{\frac{\beta}{m c^{2}} d e+\frac{e}{m c^{2}} d \beta-d a+\frac{4 \pi(m c)^{3} g_{s}}{3 h^{3}}\left[\frac{J_{40}}{n} d \beta-\right.\right. \\
& \left.\left.\frac{\beta J_{40}}{n^{2}} d n+\frac{\beta}{n}\left(\frac{\partial J_{40}}{\partial a} d a+\frac{\partial J_{40}}{\partial \beta} d \beta\right)\right]\right\}
\end{aligned}
$$

e utilizando as propriedades das derivadas das funções $J$, ficamos com

$$
d \sigma_{E}=\frac{k \beta}{m c^{2}}\left(d e-\frac{p}{n^{2}} d n\right)
$$

ou ainda, usando $\rho=n e$,

$$
d \sigma_{E}=\frac{k \beta}{m c^{2}}\left(\frac{d \rho}{n}-\frac{p+\rho}{n^{2}} d n\right)
$$

que, comparada com a relação de Gibbs (3.10), fornece

$$
\beta=\frac{m c^{2}}{k T}
$$

Com a determinação de $\beta$ podemos escrever $\sigma_{E}$ como

$$
\sigma_{E}=\frac{p+\rho}{n T}-a k
$$

que comparada com a relação de Euler (3.12), fornece

$$
a=\frac{\mu_{E}}{k T}
$$

em $\mu_{E}$ é o potencial químico em equilíbrio.

Agora que determinamos os parâmetros $\beta$ e $a$, podemos escrever a função distribuição de equilíbrio:

$$
f^{0}=\frac{g_{s} / h^{3}}{e^{-\frac{\mu_{E}}{k T}+\frac{U^{\mu} p_{\mu}}{k T}}-\varepsilon},
$$

em que $\varepsilon=0,-1,+1$ para estatística de Maxwell-Boltzmann, Fermi-Dirac e BoseEinstein, respectivamente. As expressões acima foram obtidas por Jüttner em 1911 e $1928[45,46]$. 


\subsection{Equação de Boltzmann na métrica FLRW}

Nessa seção estudaremos uma das aplicações da equação de Boltzmann na Relatividade Geral. Por razões que ficarão mais claras adiante, escreveremos o quadrimomento em letras maiúsculas, $P^{\mu}$.

Na Relatividade Geral, pode-se mostrar que o volume invariante no espaço dos momentos é $\sqrt{g} \frac{d^{3} P}{P_{0}}$, em que $g=-\operatorname{det}\left(g_{\mu \nu}\right)$ é o determinante da métrica. Além disso, os símbolos de Christoffel, diferente do espaço de Minkowski, são não nulos. Portanto, a equação de Boltzmann toma a forma

$$
\begin{aligned}
P^{\mu} \frac{\partial f}{\partial x^{\mu}}-\Gamma_{\alpha \beta}^{\mu} P^{\alpha} P^{\beta} \frac{\partial f}{\partial P^{\mu}}= & \int\left[f^{\prime} f_{1}^{\prime}\left(1+\varepsilon \frac{f h^{3}}{g_{s}}\right)\left(1+\varepsilon \frac{f_{1} h^{3}}{g_{s}}\right)\right. \\
& \left.-f f_{1}\left(1+\varepsilon \frac{f^{\prime} h^{3}}{g_{s}}\right)\left(1+\varepsilon \frac{f_{1}^{\prime} h^{3}}{g_{s}}\right)\right] \times \\
& W\left(P, P_{1} \mid P^{\prime}, P_{1}^{\prime}\right) \sqrt{g} \frac{d^{3} P_{1}}{P_{1}^{0}} \sqrt{g} \frac{d^{3} P^{\prime}}{P^{\prime 0}} \sqrt{g} \frac{d^{3} P_{1}^{\prime}}{P_{1}^{\prime 0}} .
\end{aligned}
$$

Passaremos a equação acima para a camada de massa ("mass-shell"). Para isso, usaremos as seguintes relações entre derivadas [53]:

$$
\frac{\partial f}{\partial x^{\mu}}=\frac{\partial f}{\partial x^{\mu}}+\frac{\partial f}{\partial P^{0}} \frac{\partial P^{0}}{\partial x^{\mu}}, \quad \frac{\partial f}{\partial P^{i}}=\frac{\partial f}{\partial P^{i}}+\frac{\partial f}{\partial P^{0}} \frac{\partial P^{0}}{\partial P^{i}}
$$

em que do lado esquerdo a função de distribuição é uma função de $f\left(x^{\mu}, P^{i}\right)$, enquanto que do lado direito é uma função de $f\left(x^{\mu}, P^{\mu}\right)$. Da condição da camada de massa $\left(g_{\mu \nu} P^{\mu} P^{\nu}=\right.$ $m^{2}$ ), tiramos as seguintes relações:

$$
\frac{\partial P^{0}}{\partial x^{\mu}}=-\frac{1}{P_{0}} P^{\nu} P_{\alpha} \Gamma_{\mu \nu}^{\alpha}, \quad \frac{\partial P^{0}}{\partial P^{i}}=-\frac{P_{i}}{P_{0}} .
$$

Com isso, podemos escrever a equação de Boltzmann na camada de massa:

$$
P^{\mu} \frac{\partial f}{\partial x^{\mu}}-\Gamma_{\alpha \beta}^{i} P^{\alpha} P^{\beta} \frac{\partial f}{\partial P^{i}}=\mathcal{C}[f]
$$

em que $\mathcal{C}[f]$ representa o termo colisional.

Substituindo os símbolos de Christoffel para a métrica FLRW na parte esquerda da equação acima, obtemos 


$$
E\left(\frac{\partial f}{\partial t}-2 \frac{\dot{a}}{a} P^{i} \frac{\partial f}{\partial P^{i}}\right)=\mathcal{C}[f],
$$

em que usamos $E=P^{0}=P_{0}$ por simplicidade.

Aqui é importante introduzir o conceito de momento físico $\left(p^{i}\right)$, que, assim como coordenadas físicas e comóveis, está relacionado ao momento comóvel $\left(P^{i}\right)$ através do fator de escala, $p^{i}=a P^{i}$. Feita a troca de variáveis, a equação fica

$$
\frac{\partial f}{\partial t}-\frac{\dot{a}}{a} p^{i} \frac{\partial f}{\partial p^{i}}=\frac{1}{E} \mathcal{C}[f]
$$

Vimos que tanto para situações sem colisões quanto em equilíbrio, o lado direito da equação de Boltzmann se anula, simplificando a equação de Boltzmann para

$$
\frac{\partial f}{\partial t}-\frac{\dot{a}}{a} p^{i} \frac{\partial f}{\partial p^{i}}=0
$$

Lançando mão da condição de isotropia, podemos escrever a função de distribuição como $f=f(p, t)$ e a equação de Boltzmann em termos do módulo do momento:

$$
\frac{\partial f}{\partial t}-\frac{\dot{a}}{a} p \frac{\partial f}{\partial p}=0
$$

\subsubsection{Equações de Balanço}

O fluxo de partículas, o fluxo de entropia e o tensor de energia-momento são definidos por

$$
\begin{aligned}
N^{\mu} & =\int f P^{\mu} \sqrt{g} \frac{d^{3} P}{P^{0}}=\int f P^{\mu} a^{3} \frac{d^{3} P}{P^{0}} \\
S^{\mu} & =-\int\left(f \ln \left(\frac{f h^{3}}{g}\right)-f\right) P^{\mu} \sqrt{g} \frac{d^{3} P}{P^{0}} \\
& =-\int\left(f \ln \left(\frac{f h^{3}}{g}\right)-f\right) P^{\mu} a^{3} \frac{d^{3} P}{P^{0}}, \\
T^{\mu \nu} & =\int f P^{\mu} P^{\nu} \sqrt{g} \frac{d^{3} P}{P^{0}}=\int f P^{\mu} P^{\nu} a^{3} \frac{d^{3} P}{P^{0}} .
\end{aligned}
$$


Observamos que, devido à isotropia do espaço, as únicas componentes não nulas de $N^{\mu} \mathrm{e}$ $S^{\mu}$ são dadas por

$$
\begin{aligned}
N^{0}=n & =\int f a^{3} d^{3} P \\
S^{0}=s & =-\int(f \ln f-f) a^{3} d^{3} P .
\end{aligned}
$$

A pressão hidrostática pode ser obtida através do tensor de energia-momento por

$$
\begin{aligned}
\mathbf{P} & =-\frac{1}{3} g_{i j} T^{i j}=-\frac{1}{3} g_{i j} \int f \frac{P^{i} P^{j}}{E} a^{3} d^{3} P \\
& =-\frac{1}{3} g_{i j} \int f \frac{p^{i} p^{j}}{a^{2} E} d^{3} p=\frac{1}{3} \delta_{i j} \int f \frac{p^{i} p^{j}}{E} d^{3} p,
\end{aligned}
$$

em que na última linha $E$ é escrito em termos dos momentos $p^{i}$ e $d^{3} p=a^{3} d^{3} P$.

Antes de prosseguirmos com o tópico desta seção, lembremos que o divergente de um campo vetorial é

$$
A_{; \mu}^{\mu}=\frac{1}{g^{1 / 2}} \frac{\partial}{\partial x^{\mu}}\left(g^{1 / 2} A^{\mu}\right)
$$

em que $g=-\operatorname{det}\left(g_{\mu \nu}\right)$. No caso da métrica de FLRW, $g=a^{6}$.

Já para um tensor de dois índices, temos que

$$
B^{\mu \nu} ;_{\mu}=\frac{1}{g^{1 / 2}} \frac{\partial}{\partial x^{\mu}}\left(g^{1 / 2} B^{\mu \nu}\right)+\Gamma_{\mu \lambda}^{\nu} B^{\mu \lambda}
$$

em que $\Gamma_{\mu \lambda}^{\nu}$ são as conexões da métrica.

Agora, calcularemos algumas integrais que serão importantes nos passos a seguir e em capítulos posteriores. São elas:

$$
\int p \frac{\partial f}{\partial p} d^{3} p=4 \pi \int p^{3} \frac{\partial f}{\partial p} d p=\left.4 \pi f p^{3}\right|_{0} ^{\infty}-3 \int f d^{3} p=-3 \int f d^{3} p
$$

em que o primeiro termo da integração por partes se cancela pelo fato de que $p^{3}$ é rapidamente suprimido por $f$ para altos valores de $p$.

Outra integral importante é

$$
\begin{aligned}
\int E p \frac{\partial f}{\partial p} d^{3} p & =4 \pi \int E p^{3} \frac{\partial f}{\partial p} d p=4 \pi\left\{\left.E p^{3} f\right|_{0} ^{\infty}-\int f\left(\frac{p^{4}}{E}+3 E p^{2}\right) d p\right\} \\
& =-3(\rho+\mathbf{P}) .
\end{aligned}
$$


A última integral a ser computada é

$$
\begin{aligned}
& \int p \frac{\partial f}{\partial p} \ln f d^{3} p=4 \pi \int p^{3} \frac{\partial f}{\partial p} \ln f d p \\
& =\left.4 \pi p^{3} f \ln f\right|_{0} ^{\infty}-4 \pi \int f\left(3 p^{2} \ln f+\frac{p^{3}}{f} \frac{\partial f}{\partial p}\right) d p \\
& =-\int f \ln f d^{3} p-4 \pi \int p^{3} \frac{\partial f}{\partial p} d p \\
& =-3 \int(f \ln f-f) d^{3} p \text {. }
\end{aligned}
$$

A partir da equação de Boltzmann, podemos verificar importantes resultados da termodinâmica. Para isso, calcularemos o divergente das quantidades definidas no começo dessa seção.

- Fluxo de Partículas

$$
\begin{aligned}
N_{;_{\mu}} & =\frac{1}{a^{3}} \frac{\partial}{\partial t}\left(a^{3} \int f d^{3} p\right) \\
& =3 \frac{\dot{a}}{a} \int f d^{3} p+\int \frac{\partial f}{\partial t} d^{3} p \\
& =3 \frac{\dot{a}}{a} \int f d^{3} p+\frac{\dot{a}}{a} \int p^{i} \frac{\partial f}{\partial p^{i}} d^{3} p \\
& =3 \frac{\dot{a}}{a} \int f d^{3} p-3 \frac{\dot{a}}{a} \int f d^{3} p=0
\end{aligned}
$$

em que na penúltima passagem foi utilizada a equação de Boltzmann (2.73) e na última foi feita uma integração por partes.

- Fluxo de Entropia

$$
\begin{aligned}
S_{; \mu}^{\mu} & =\frac{1}{a^{3}} \frac{\partial}{\partial x^{\mu}}\left(a^{3} \int(f-f \ln f) P^{\mu} a^{3} \frac{d^{3} P}{P^{0}}\right) \\
& =\frac{1}{a^{3}} \frac{\partial}{\partial x^{\mu}}\left(a^{3} \int(f-f \ln f) d^{3} p\right) \\
& =3 H \int(f-f \ln f) d^{3} p-\int\left(\frac{\partial f}{\partial t} \ln f\right) d^{3} p \\
& =3 H \int(f-f \ln f) d^{3} p-H \int p \frac{\partial f}{\partial p} \ln f d^{3} p=0
\end{aligned}
$$


- Tensor Energia-Momento

Por fim, calcularemos a projeção sobre $u_{\mu}$, de um observador comóvel, do tensor energia-momento

$$
\begin{aligned}
u_{\mu} T^{\mu \nu}{ }_{i \nu} & =\frac{1}{a^{3}} u_{\mu} \frac{\partial}{\partial x^{\nu}}\left(a^{3} \int f P^{\mu} P^{\nu} a^{3} \frac{d^{3} P}{P^{0}}\right)+u_{\mu} \Gamma_{\nu \lambda}^{\mu} \int f P^{\nu} P^{\lambda} a^{3} \frac{d^{3} P}{P^{0}} \\
& =\frac{1}{a^{3}} \frac{\partial}{\partial t}\left(a^{3} \int f E d^{3} p\right)+\Gamma_{\nu \lambda}^{0} \int f P^{\nu} P^{\lambda} a^{3} \frac{d^{3} P}{P^{0}} \\
& =3 H \int f E d^{3} p+\int \frac{\partial f}{\partial t} E d^{3} p+H \delta_{i j} \int f \frac{p^{i} p^{j}}{E} d^{3} p \\
& =3 H(\rho+\mathbf{P})+H \int p \frac{\partial f}{\partial p} E d^{3} p \\
& =3 H(\rho+\mathbf{P})-3 H(\rho+\mathbf{P})=0
\end{aligned}
$$

Portanto, utilizando a equação de Boltzmann (2.73) ao calcular o divergente das quantidades físicas definidas na teoria cinética, obtemos as equações de balanço da termodinâmica de equilíbrio (sem criação de partículas).

\subsubsection{Lei de Evolução da Temperatura}

Utilizando a equação de Boltzmann podemos obter a lei de temperatura de um fluido que evolui livremente em um Universo em expansão. Para isso, vamos reescrever (sem perda de generalidade para os resultados que obteremos) a função distribuição em um referencial comóvel da seguinte maneira:

$$
f^{0}=e^{\alpha(t)-\beta(t) E}
$$

Substituindo a função acima na equação (2.74) ficamos com

$$
\frac{\dot{\alpha}}{\dot{\beta}}=E-\frac{\dot{a}}{a} \frac{\beta}{\dot{\beta}} \frac{p^{2}}{E} .
$$

A equação acima não pode ser resolvida para quaisquer $\alpha$ e $\beta$. Porém, podemos estudar dois importantes casos limites, $m \rightarrow 0$ e $m \rightarrow \infty$. No primeiro caso, temos que $E=p$ e a equação fica:

$$
\frac{\dot{\alpha}}{\dot{\beta}}=p\left(1-\frac{\dot{a}}{a} \frac{\beta}{\dot{\beta}}\right) .
$$

Para partículas sem massa, o potencial químico é zero. Portanto, $\alpha=0$ é parte da 
solução da equação acima. Como pode-se verificar, $\beta \propto a$ zera o lado direito da equação. Esse é um importante resultado, a saber, a temperatura de um gás de partículas sem massa que expande livremente é inversamente proporcional ao fator de escala,

$$
T \propto \frac{1}{a}
$$

Para o outro caso limite, $m \rightarrow \infty$ ou $m \gg p$, temos que $E=m\left(1+\frac{p^{2}}{2 m}\right)$ e a equação a ser resolvida é

$$
\frac{\dot{\alpha}}{\dot{\beta}}-m=\frac{p^{2}}{2 m}-\frac{\dot{a}}{a} \frac{\beta}{\dot{\beta}} \frac{p^{2}}{m},
$$

que tem soluções $\alpha-m \beta=$ const e $\frac{\dot{a}}{a} \frac{\beta}{\beta}=\frac{1}{2}$. Isso significa que para partículas nãorelativísticas a temperatura evolui com o inverso do quadrado do fator de escala

$$
T \propto \frac{1}{a^{2}}
$$

\subsubsection{Equação de Evolução de Relíquias Cósmicas}

Como vimos anteriormente, o Universo passou por diferentes fases. Logo após o Big Bang, o Universo era formado por um plasma primordial (radiação, matéria, matéria escura etc), uma verdadeira sopa cósmica. No início, a temperatura do Universo era muito alta e as componentes desse plasma eram mantidas em equilíbrio. Com a expansão do Universo, a temperatura e a energia foram diminuindo e cada componente do plasma original passa a se comportar de forma diferente. Por exemplo, à medida em que a temperatura do meio abaixa, o plasma não tinha mais energia para gerar partículas massivas através de reações de aniquilação e produção por pares. Quando a taxa de produção pelo plasma se torna insignificante e a componente para de ser afetada pelo plasma, a componente se desacopla e passa a evoluir independentemente. Se as componentes do plasma continuassem a evoluir em equilíbrio com o plasma, não haveria o desacoplamento e não teriam sido formadas estruturas como átomos, moléculas, galáxias etc.

Podemos utilizar a equação de Boltzmann com colisões (2.72) para ter uma descrição analítica do quadro apresentado acima. Para isso, multiplicamos por $\frac{g}{(2 \pi)^{3}} d^{3} p$, integramos a equação para se obter

$$
\frac{d n}{d t}+3 \frac{\dot{a}}{a} n=\frac{g}{(2 \pi)^{3}} \int \mathcal{C}[f] \frac{d^{3} p}{E}
$$


em que $g$ é o número de graus de liberdade da partícula em questão e $n$ é a densidade, definida por

$$
n=\frac{g}{(2 \pi)^{3}} \int f d^{3} p
$$

No Apêndice B, seguimos os passos de [21] para determinar o lado direito da equação (2.95), ficando com a equação de evolução de uma relíquia cósmica

$$
\dot{n}+3 H n=-<\sigma v>\left(n^{2}-n_{e q}^{2}\right)
$$

em que $n_{e q}$ é a densidade da espécie quando está em equilíbrio e $<\sigma v>$ é a seção de choque de aniquilação.

Antes de prosseguirmos com a análise da equação de evolução, estabeleceremos dois limites importantes para $n_{e q}$. Estaremos interessados em casos em que as partículas desacoplam do plasma quando a temperatura do meio é menor que $E-\mu$. Dessa maneira, podemos utilizar a função distribuição de Maxwell-Boltzmann. Assim, temos os seguintes limites:

$$
n_{e q} \equiv \frac{g}{(2 \pi)^{3}} \int e^{-E / T} d^{3} p=\left\{\begin{array}{ll}
g\left(\frac{m T}{2 \pi}\right)^{3 / 2} e^{-m / T} & \text { para } m \gg T \\
g \frac{T^{3}}{\pi^{2}} & \text { para } m \ll T
\end{array} .\right.
$$

Da expressão acima, podemos ver que se a espécie se mantivesse sempre em equilíbrio com o plasma, no limite $m \gg T$, sua densidade cairia exponencialmente e não haveria relíquias para serem medidas hoje em dia.

Vamos reescrever a equação de evolução em termos de uma nova variável, definida como $Y=\frac{n}{s}$, em que $n$ é o número de partículas e $s$ é a densidade de entropia. Supondo que a entropia total, $S=a^{3} s$, se mantém constante, temos que a densidade de entropia varia como

$$
\dot{s}=-3 \frac{\dot{a}}{a} s .
$$

O processo de desacoplamento da componente do fluido cósmico é chamado de freeze-out e ocorre na era da radiação. A densidade de entropia e energia nesse regime são dadas por

$$
s=\frac{2 \pi^{2}}{45} g_{\star, S} T^{3} \quad \text { e } \quad \rho=\frac{\pi^{2}}{30} g_{\star} T^{4}
$$

em que $g_{\star, S}$ e $g_{\star}$ contam diferentes graus de liberdade relativísticos.

Vamos mudar a variável independente de $t$ para $x=\frac{m}{T}$, em que $m$ é a massa da relíqua 
em estudo e $T$ é a temperatura. Além disso, suporemos o caso mais simples em que os graus de liberdade não mudam com a temperatura, ou seja, $g=g_{\star, S}=g_{\star}$. Com isso, a equação de evolução toma a forma

$$
\frac{d Y}{d x}=-\frac{\lambda}{x^{2}}\left(Y^{2}-Y_{e q}^{2}\right)
$$

em que $Y_{e q}=n_{e q} / s$ e $\lambda=0.264 m m_{p l} \sqrt{g}\left\langle\sigma v>\right.$, em que $m_{p l}$ é a massa de Planck.

A Figura 2.1 mostra a equação de evolução para diferentes valores de $\lambda$. Mantendo a massa da partícula fixa, vemos que o aumento da seção de choque (aumentando o valor de $\lambda$ ) leva a um freeze-out ocorrendo mais tarde e com abundância menor.

O processo descrito nessa seção é uma importante ferramenta para a determinação de parâmetros cosmológicos como, por exemplo, massa e seção de choque de candidatos a matéria escura.

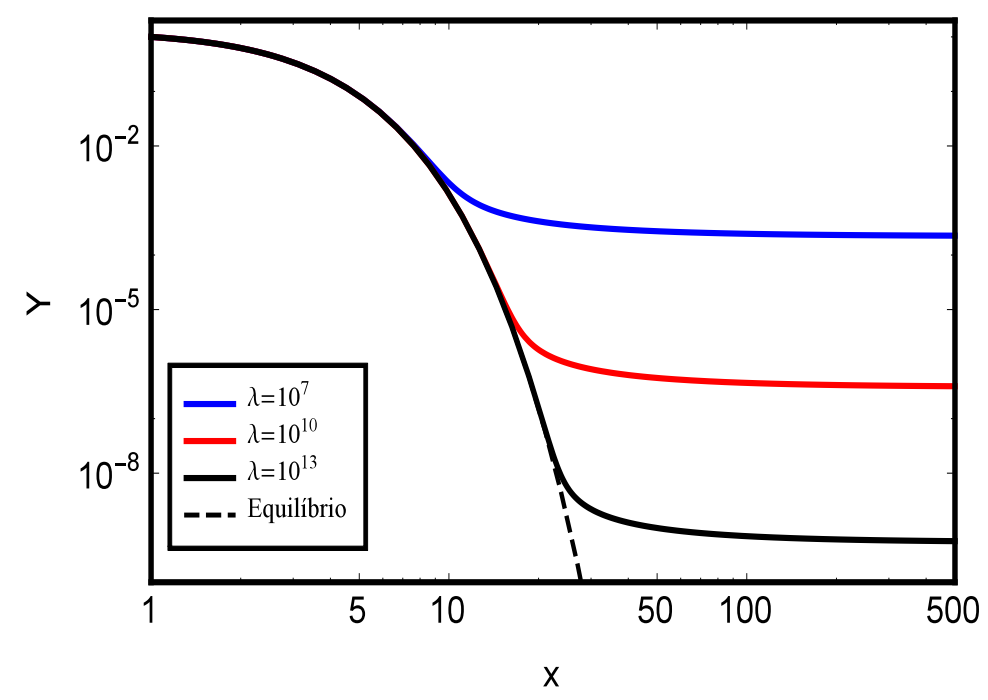

Figura 2.1: Solução da equação de evolução para diferentes valores de $\lambda$. 


\section{Capítulo 3}

\section{Criação Gravitacional de Partículas:}

Formulação Termodinâmica e

\section{Cosmologia}

O Modelo Padrão da Cosmologia (MPC) contém alguns ingredientes fundamentais: o Universo é praticamente plano; a idade do Universo deve ser da ordem de 14 bilhões de anos (na verdade, ser, no mínimo, mais velho que suas estruturas mais antigas, os aglomerados de galáxias que têm idades da ordem de 12,7 bilhões de anos); existe alguma matéria escura não bariônica e o Universo está em expansão acelerada.

O mecanismo mais comum usado para explicar a aceleração do Universo é a existência da constante cosmológica, que age nas equações de Einstein como um fluido com equação de estado $p=-\rho$.

Apesar do sucesso em explicar os dados observacionais, a existência da constante cosmológica enfrenta grandes problemas teóricos. Por exemplo, a diferença entre a densidade medida e a calculada pela teoria quântica de campos varia de 50-120 ordens de grandeza. Por esse motivo, vários outros modelos foram propostos para explicar a expansão acelerada do Universo. Se assumirmos que não exista a constante cosmológica, precisaremos de um mecanismo capaz de acelerar o Universo.

Um modelo proposto recentemente, que consegue explicar a aceleração atual do Universo, é o de criação de partículas. À custa do campo gravitacional, partículas são criadas a todo momento. Parker e colaboradores desenvolveram uma descrição microscópica para o mecanismo de produção de partículas através de um campo gravitacional que varia no 
tempo em um Universo em expansão [56, 57]. Tais estudos foram baseados na técnica Bogoliubov mode-mixing no contexto da Teoria Quântica de Campos (TQC) em espaços curvos.

Podemos entender esse mecanismo quântico de criação gravitacional de partículas da seguinte maneira. No caso de um campo escalar que evolui numa geometria FLRW, por exemplo, sua massa efetiva passa a ser uma quantidade dependente do tempo. Quando o campo é quantizado, leva à criação de partículas com a energia das novas partículas sendo suprida pelo campo gravitacional de fundo que varia no tempo [58]. Em outras palavras, diferente do espaço-tempo de Minkowski, uma geometria que varia no tempo se comporta como uma "bomba" transformando curvatura em partículas. Apesar de descrever rigorosamente o processo de criação de partículas para campos teste que evoluem no fundo cósmico, essa abordagem não provê uma receita clara de como a matéria criada modifica as equações de Einstein. Esse é o problema de back reaction que ainda não foi resolvido.

A criação de partículas no espaço tempo à custa do campo gravitacional foi descrita macroscopicamente por Prigogine e colaboradores. O trabalho foi baseado na termodinâmica de não-equilíbrio para sistemas abertos [15]. Essa abordagem foi revista por alguns autores [59] dentro de uma formulação manifestamente covariante e, em seguida, aplicada na Cosmologia. A vantagem dessa segunda abordagem é que o problema de back reaction é naturalmente incorporado e restrito pela segunda lei da Termodinâmica. Contudo, também é uma descrição incompleta no sentido de que a taxa de criação de partículas deve ser calculada a partir da TQC em uma geometria de FLRW. A diferença entre o mecanismo de criação de partículas e o mecanismo com viscosidade volumétrica foi discutido por $[16,60]$. Eles demonstraram que a produção de partículas e viscosidade volumétrica são capazes de gerar a mesma dinâmica cósmica, porém os processos são completamentes diferentes do ponto de vista termodinâmico.

Nos últimos anos, muita atenção tem sido dada para cosmologias ditadas por um processo de criação "adiabático" de partículas em que matéria e entropia são geradas mas a entropia específica (por partícula) continua constante. Como o processo de criação é descrito macroscopicamente por uma pressão negativa de origem quântica, esses modelos têm sido investigados como uma possibilidade de unificação do chamado setor escuro do Universo. Nesse caso, o estágio acelerado do Universo se torna uma consequência da 
produção gravitacional de partículas $[61,62,63,64]$. Mais recentemente, uma cosmologia em que o espaço, tempo e matéria evoluem entre fases de Sitter primordiais e atuais compelidos por produção de partículas foi proposta e suas predições comparadas com dados disponíveis $[3,65,66]$. A consistência de tal cenário com a segunda lei da Termodinâmica generalizada foi discutida em detalhes em [67].

\subsection{Termodinâmica do Modelo}

A termodinâmica do modelo de criação de matéria será discutida como apresentada em [59].

Considere que o fluido que está constantemente sendo criado possua um tensor de energia-momento da forma

$$
T^{\alpha \beta}=T_{E}^{\alpha \beta}+\Delta T^{\alpha \beta}
$$

em que

$$
T_{E}^{\alpha \beta}=(\rho+\mathbf{P}) u^{\alpha} u^{\beta}-\mathbf{P} g^{\alpha \beta}
$$

é o tensor de energia-momento de equilíbrio, $\rho$ é a densidade de energia, $\mathbf{P}$ é a pressão de equilíbrio e $\Delta T^{\alpha \beta}$ é uma correção que tem a seguinte forma:

$$
\Delta T^{\alpha \beta}=-\mathbf{P}_{\mathbf{c}} h^{\alpha \beta}
$$

em que $\mathbf{P}_{\mathbf{c}}$ é a pressão que surge do processo de criação de partículas e $h^{\alpha \beta}=\left(g^{\alpha \beta}-u^{\alpha} u^{\beta}\right)$ é o operador que projeta um tensor em outro perpendicular a $u^{\alpha}$, chamado de projetor. De tal forma que a conservação do tensor de energia-momento resulta em

$$
\dot{\rho}+\Theta\left(\rho+\mathbf{P}+\mathbf{P}_{\mathbf{c}}\right)=0
$$

em que $\Theta=u^{\alpha}{ }_{;}=3 \frac{\dot{a}}{a}$ é a expansão do fluido.

Aqui podemos ver, a partir da equação acima, que uma pressão de criação de partículas é negativa. Para isso, vamos analisar a equação em um dado volume $V=a^{3}$. A expansão do fluido está relacionada com a variação do volume por $3 \frac{\dot{a}}{a}=\frac{\dot{V}}{V}$. Com isso, podemos reescrever a equação de conservação da seguinte maneira: 


$$
\frac{d}{d t}(\rho V)=-\left(\mathbf{P}+\mathbf{P}_{\mathbf{c}}\right) \frac{d V}{d t}
$$

Considerando um volume em expansão, $\frac{d V}{d t}>0$, com matéria sendo injetada, podemos concluir que a taxa em que a energia $(\rho V)$ nesse volume cai, devido à expansão, é menor que na situação sem criação de matéria. Isso se deve ao fato de existir mais matéria (mais energia) que no caso sem criação. Portanto, da expressão acima, vemos que a pressão total no caso com a criação de matéria, $\mathbf{P}+\mathbf{P}_{\mathbf{c}}$, tem que ser menor que a pressão $\mathbf{P}$, do caso sem criação, para que $\rho V$ diminua mais lentamente. Como a pressão de equilíbrio é sempre positiva, a única forma de $\mathbf{P}+\mathbf{P}_{\mathbf{c}}<\mathbf{P}$ é $\mathbf{P}_{\mathbf{c}}<0$.

O tensor fluxo de partícula tem a forma

$$
N^{\alpha}=n u^{\alpha}
$$

em que $n$ é a densidade de partículas. Como o modelo assume que partículas estão sendo criadas, a equação de balanço é

$$
N^{\alpha} ; \alpha=\Psi
$$

em que $\Psi$ é a fonte de partículas.

O tensor fluxo de entropia é

$$
S^{\alpha}=n \sigma u^{\alpha}
$$

em que $\sigma$ é a entropia específica (por partícula) e satisfaz a segunda lei da termodinâmica

$$
S^{\alpha} ; \alpha \geq 0
$$

Para esse sistema, a relação de Gibbs é

$$
n T d \sigma=d \rho-\frac{\rho+\mathbf{P}}{n} d n
$$

em que $T$ é a temperatura do sistema. Da expressão acima é possível mostrar que

$$
S^{\alpha} ; \alpha=-\frac{\mathbf{P}_{\mathbf{c}} \Theta}{T}-\frac{\mu \Psi}{T}
$$


em que $\mu$ é o potencial químico definido pela relação de Euler:

$$
\mu=\frac{\rho+\mathbf{P}}{n}-T \sigma
$$

Como estamos tratando de processos em que a única diferença do processo em equilíbrio é a produção de partículas, é razoável pensar que a a pressão de criação seja da forma

$$
\mathbf{P}_{\mathbf{c}}=-\frac{\alpha \Psi}{\Theta}
$$

e com essa escolha a produção de entropia é

$$
S^{\alpha} ;_{\alpha}=\frac{\Psi}{T}(\alpha-\mu)
$$

Dessa forma, quando $\Psi=0$ a produção de entropia se anula, como é de se esperar em processos em equilíbrio. Usando a relação de Euler, podemos escrever

$$
S^{\alpha}{ }_{\alpha}=\Psi \sigma+\left(\alpha-\frac{\rho+\mathbf{P}}{n}\right) \frac{\Psi}{T}
$$

que podemos comparar com a diferenciação da expressão do fluxo de entropia, $S^{\alpha}{ }_{\alpha}=$ $\Psi \sigma+n \dot{\sigma}$, e obter

$$
\dot{\sigma}=\frac{\Psi}{n T}\left(\alpha-\frac{\rho+\mathbf{P}}{n}\right) .
$$

No caso de processos em que a criação de partículas é adiabática, temos que $\dot{\sigma}=0$ e

$$
\mathbf{P}_{\mathbf{c}}=-\frac{\rho+\mathbf{P}}{n \Theta} \Psi
$$

A evolução da temperatura para um sistema com criação de matéria é [59]

$$
\frac{\dot{T}}{T}=-\left(\frac{\partial \mathbf{P}}{\partial \rho}\right)_{n} \Theta-\frac{\mathbf{P}_{\mathbf{c}} \Theta+(\partial \rho / \partial n)_{T} \Psi}{T(\partial \rho / \partial T)_{n}}
$$

Considerando $\dot{\sigma}=0$, a equação acima resulta em

$$
\frac{\dot{T}}{T}=\left(\frac{\partial \mathbf{P}}{\partial \rho}\right)_{n} \frac{\dot{n}}{n}=\left(\frac{\partial \mathbf{P}}{\partial \rho}\right)_{n}\left(\frac{\Psi}{n}-\Theta\right) .
$$

que se reduz à lei de equilíbrio no limite $\Psi \rightarrow 0$. Em particular, para partículas ultrare- 
lativísticas, $\left(\frac{\partial \mathbf{P}}{\partial \rho}\right)_{n}=\frac{1}{3}$, a lei acima fornece

$$
\frac{\dot{T}}{T}=-\frac{\dot{a}}{a}+\frac{1}{3} \frac{\Psi}{n}
$$

\subsection{Cosmologia com Criação de Matéria Escura}

Em um Universo com uma métrica FLRW, as equações de campo de Einstein para o modelo com criação de matéria escura são [61, 64]

$$
\begin{aligned}
8 \pi G \rho & =3 \frac{\dot{a}^{2}}{a^{2}}+3 \frac{\kappa}{a^{2}}, \\
8 \pi G\left(\mathbf{P}+\mathbf{P}_{\mathbf{c}}\right) & =-2 \frac{\ddot{a}}{a}-\frac{\dot{a}^{2}}{a^{2}}-\frac{\kappa}{a^{2}}, \\
\frac{\dot{n}}{n}+3 \frac{\dot{a}}{a} & =\frac{\psi}{n} \equiv \Gamma,
\end{aligned}
$$

em que $\rho, \mathbf{P}, \mathbf{P}_{\mathbf{c}}, n$ e $\psi$ são, respectivamente, densidade de energia, pressão termodinâmica, pressão de criação, densidade do número de partículas e a taxa de criação de partículas. A quantidade $\Gamma$ é a taxa de criação do processo.

Como vimos na seção anterior, se a criação for de maneira que o processo seja adiabático, a pressão de criação é

$$
\mathbf{P}_{\mathbf{c}}=-\frac{\rho+\mathbf{P}}{3 n H} \Psi \equiv-\frac{\rho+\mathbf{P}}{3 H} \Gamma
$$

Utilizando a equação de estado usual, $\mathbf{P}=\omega \rho$, pode-se obter a equação que governa a evolução do fator de escala:

$$
a \ddot{a}+\left[\frac{1+3 \omega}{2}-\frac{(1+\omega) \Gamma}{2 H}\right]\left(\dot{a}^{2}+\kappa\right)=0 .
$$

Para um Universo plano e com matéria escura fria $(\kappa=\omega=0)$, a equação acima é reduzida a

$$
a \ddot{a}+\frac{1}{2}\left(1-\frac{\Gamma}{H}\right) \dot{a}^{2}=0,
$$

ou ainda, podemos obter uma equação para a evolução do parâmetro de Hubble:

$$
\dot{H}+\frac{3}{2} H^{2}\left(1-\frac{\Gamma}{3 H}\right)=0
$$


Para continuarmos os estudos do modelo com criação de matéria escura, precisamos de conhecer a forma de $\Gamma$. Como proposto em [61, 64], definimos

$$
\Gamma=3 \gamma H_{0}+3 \beta H
$$

em que $\gamma$ e $\beta$ são parâmetros que estão no intervalo $[0,1]$. $H_{0}$ é a constante de Hubble.

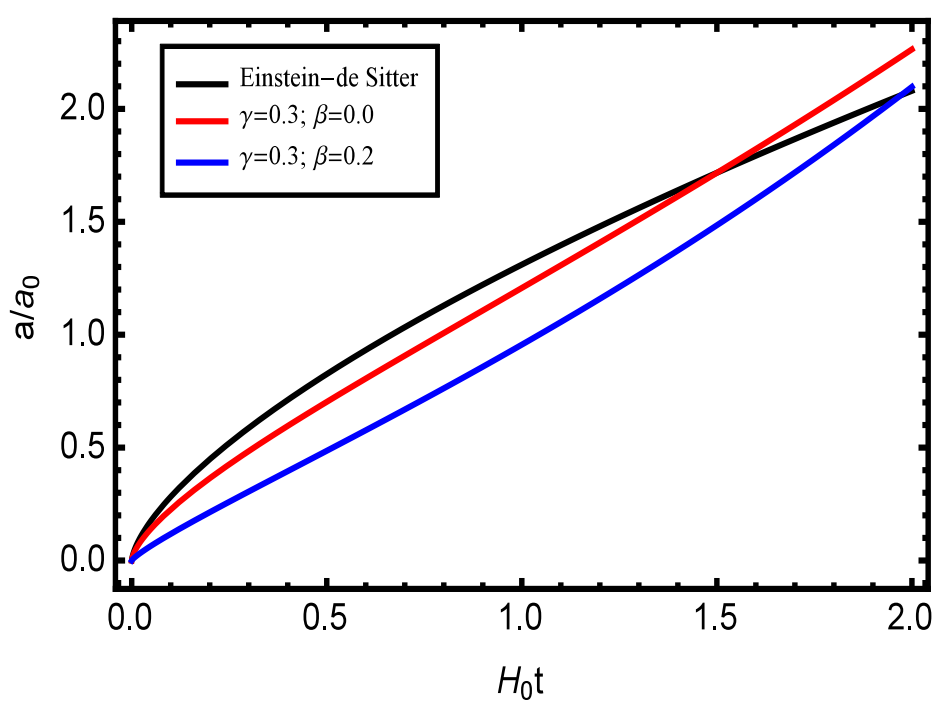

Figura 3.1: Fator de escala para $\gamma=0.3$ e dois valores distintos de $\beta$. Os resultados são comparados com o modelo Einstein-de Sitter.

Inserindo a definição acima na equação de evolução de $H$, encontramos

$$
\dot{H}+\frac{3}{2} H^{2}\left(1-\beta-\frac{\gamma H_{0}}{H}\right)=0
$$

que tem solução

$$
H(t)=H_{0}\left(\frac{\gamma}{1-\beta}\right) \frac{e^{\frac{3 \gamma H_{0}}{2} t}}{\left(e^{\frac{3 \gamma H_{0}}{2} t}-1\right)},
$$

que pode ser integrada para se obter a evolução do fator de escala:

$$
a(t)=a_{0}\left[\left(\frac{1-\gamma-\beta}{\gamma}\right)\left(e^{\frac{3 \gamma H_{0}}{2} t}-1\right)\right]^{\frac{2}{3(1-\beta)}}
$$

em que $a_{0}$ é o fator de escala hoje.

No limite $\gamma \rightarrow 0$, a expressão é reduzida ao caso estudado em [62]:

$$
a(t)=a_{0}\left[\frac{3}{2}(1-\beta) H_{0} t\right]^{\frac{2}{3(1-\beta)}}
$$


quando $\beta=0$, se reduz ao modelo Einstein-de Sitter. Na Figura 3.1 são mostrados fatores de escala para alguns valores de $\beta$ e $\gamma$.

Podemos calcular a idade do Universo para o modelo fazendo $a=a_{0}$ em (3.31),

$$
t_{0}=H_{0}^{-1} \frac{2}{3 \gamma} \ln \left(\frac{1-\beta}{1-\gamma-\beta}\right)
$$

A Figura 3.2 mostra a idade do Universo em função de $\gamma$ para alguns valores de $\beta$. A linha verde representa a idade de 12.7 bilhões de anos, limite inferior para a idade do Universo dado pela idade de aglomerado de galáxias. É interessante notar que o modelo de criação de matéria escura é capaz de contornar o problema da idade do Universo.

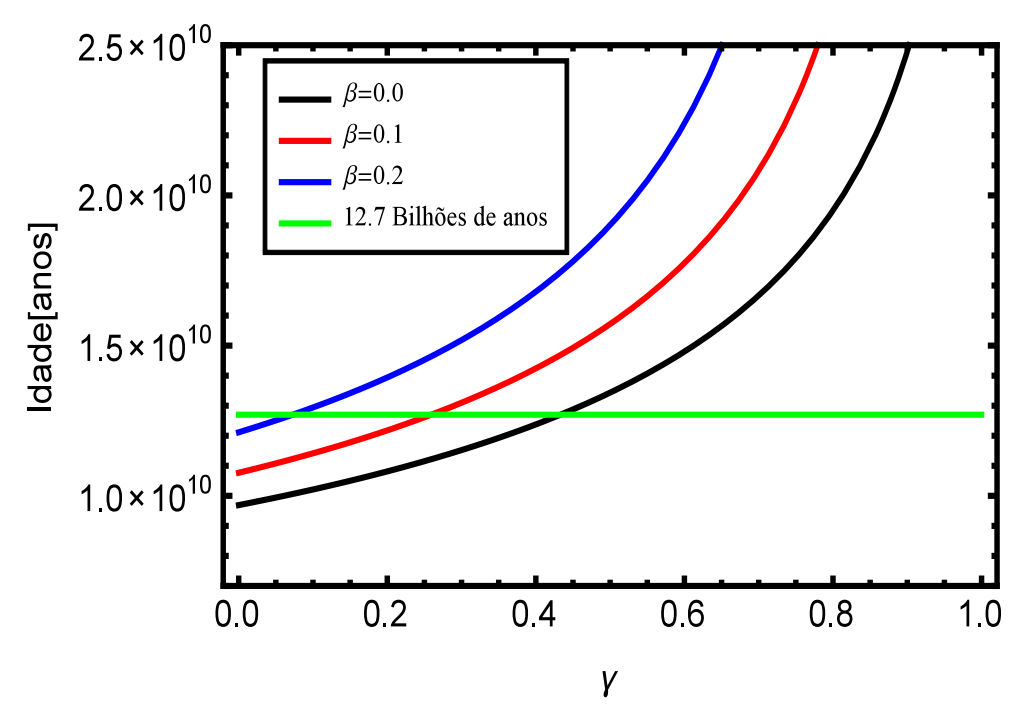

Figura 3.2: Idade do Universo em função de $\gamma$ para alguns valores de $\beta$. A linha verde representa o limite inferior para a idade do Universo.

Fazendo $\Gamma=3 \gamma H_{0}+3 \beta H$ em (3.26), podemos determinar o parâmetro de desaceleração do modelo,

$$
q=\frac{1}{2}\left[1-3 \beta-3 \gamma \frac{H_{0}}{H}\right] .
$$

Usando a definição de redshift em termos do fator de escala, podemos escrever o parâmetro de Hubble da seguinte maneira:

$$
H(z)=H_{0}\left[\frac{\gamma+(1-\gamma-\beta)(1+z)^{\frac{3}{2}(1-\beta)}}{1-\beta}\right]
$$


que, quando inserido na expressão do parâmetro de desaceleração, fornece

$$
q(z)=\frac{1}{2}\left[\frac{(1-3 \beta)(1-\gamma-\beta)(1+z)^{\frac{3}{2}(1-\beta)}-2 \gamma}{(1-\gamma-\beta)(1+z)^{\frac{3}{2}(1-\beta)}-\gamma}\right]
$$

A Figura 3.3 mostra o parâmetro de desaceleração do modelo para alguns valores de $\beta$ e $\gamma$

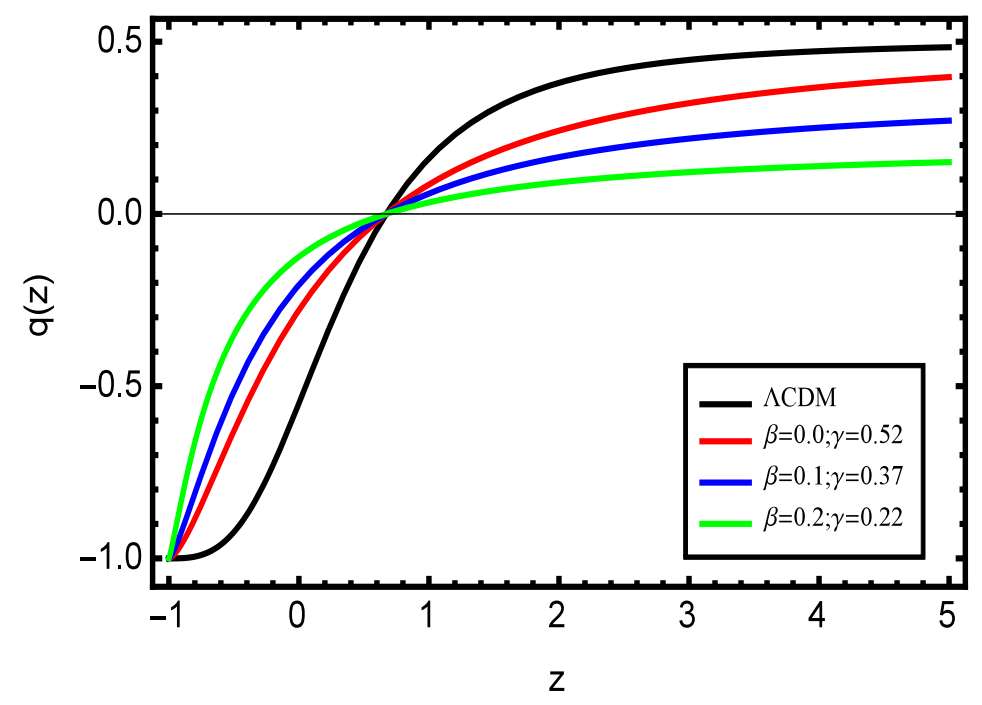

Figura 3.3: Parâmetro de desaceleração para diferentes valores de $\beta$ e $\gamma$.

\subsection{Criação de Matéria $\times \Lambda$ CDM: O Modelo LJO}

Os modelos da seção anterior, foram propostos e estudados por [61, 64]. No primeiro trabalho [61], os autores demonstraram que modelos com criação de matéria escura resolvem o problema da idade do Universo e, genericamente, são capazes de acomodar dados de observações de Supenovas Tipo Ia (SNIa). No segundo trabalho [64], bárions foram incluídos e foi testada a evolução de tais modelos em altos redshifts usando o vínculo em $z_{e q}$, o redshift da época da igualidade entre matéria e radiação, proveniente de vínculos do WMAP no efeito Integrated Sachs-Wolfe. Tal comparação revelou uma tensão entre o vínculo em $z_{e q}$ pela radiação cósmica de fundo em altos redshifts e o vínculo vindo de baixos redshifts dos dados de SNIa, desafiando a viabilidade dessa classe de modelos. Outro problema está relacionado com a relativa dificuldade matemática encontrada quando a componente bariônica é introduzida no modelo de criação de matéria proposto por [61].

A maior dificuldade de modelos com criação de matéria escura vem do fato de que 
esses modelos são espacialmente planos $\left(\Omega_{d m}+\Omega_{b a r}=1\right)$, mas não é claro como eles acomodam os dados de clusters, que consistentemente apontam para $\Omega_{d m}+\Omega_{b a r} \approx 0.3$ [68]. Como a taxa de criação de matéria afeta a quantidade de matéria atual de modo que o parâmetro de densidade de matéria medido efetivamente esteja perto daquele obtido pelas observações disponíveis?

Essas deficiências podem ser resolvidas através da uma escolha apropriada de $\Gamma$, proposta por Lima, Jesus e Oliveira (LJO) [3]. Primeiro, observamos que a aceleração da expansão do Universo é um fenômeno em baixos redshifts, ou seja, ela deve ter sido suprimida durante a fase da radiação. Isso pode ser obtido considerando que a função $\beta(t)$ seja inversamente proporcional à densidade de energia da matéria escura. Como essa quantidade é adimensional, ela pode depender de uma razão envolvendo a densidade de matéria escura. Sabemos que o efeito da criação de partículas é quantificado pela razão $\frac{\Gamma}{3 H}$. Assim, podemos garantir que o efeito da criação será desprezível para redshifts $z \geq 5$ (ver Figura (3.5)), supondo que essa razão seja inversamente proporcional à densidade de matéria escura. Mais especificamente, os autores de [3] propuseram a seguinte expressão:

$$
\frac{\Gamma}{3 H}=\alpha\left(\frac{\rho_{c 0}}{\rho_{d m}}\right)
$$

em que $\alpha$ é um parâmetro constante, $\rho_{c 0}$ é o valor da densidade crítica hoje e $\rho_{d m}$ é a densidade da matéria escura. Esse é dito modelo LJO.

A equação para evolução da densidade de energia para a matéria escura fica

$$
\dot{\rho}_{d m}+3 H \rho_{d m}=\Gamma \rho_{d m} \equiv 3 \alpha \rho_{c 0} H
$$

que pode ser integrada, resultando em

$$
\rho_{d m}=\left(\rho_{d m 0}-\alpha \rho_{c 0}\right)\left(\frac{a_{0}}{a}\right)^{3}+\alpha \rho_{c 0}
$$

ou, em termos do redshift, $1+z=a_{0} / a$,

$$
\rho_{d m}=\left(\rho_{d m 0}-\alpha \rho_{c 0}\right)(1+z)^{3}+\alpha \rho_{c 0}
$$

Como para radiação e bárions, as soluções das equações de conservação de energia são as usuais, ou seja, $\rho_{\text {rad }}=\rho_{\text {rad } 0}(1+z)^{4}$ e $\rho_{\text {bar }}=\rho_{\text {bar } 0}(1+z)^{3}$, podemos inserir essas 
expressões na equação de Friedmann, chegando em (como estamos tratando apenas do estágio da matéria, não levaremos em conta a radiação)

$$
\left(\frac{H}{H_{0}}\right)^{2}=\left(\Omega_{m}-\alpha\right)(1+z)^{3}+\alpha+\left(1-\Omega_{m}\right)(1+z)^{2},
$$

em que definimos

$$
\Omega_{m} \equiv \Omega_{d m}+\Omega_{b a r}
$$

e usamos a condição $\Omega_{k}=1-\Omega_{m}$. A similaridade da expressão acima com a do modelo $\Lambda \mathrm{CDM}$ é surpreendente, pois ela foi obtida considerando-se apenas a existência de matéria não-relativística. O parâmetro de Hubble para o modelo $\Lambda$ CDM é

$$
\left(\frac{H_{\Lambda C D M}}{H_{0}}\right)^{2}=\Omega_{m}(1+z)^{3}+\Omega_{\Lambda}+\left(1-\Omega_{m}-\Omega_{\Lambda}\right)(1+z)^{2} .
$$

Podemos ver que os modelos possuem o mesmo parâmetro de Hubble $H(z)$, com $\alpha$ fazendo o papel dinâmico de $\Omega_{\Lambda}$ e $\Omega_{m}$ sendo substituído por $\Omega_{m}-\alpha$. Tal mapeamento fica mais evidente se definirmos um parâmetro de densidade de matéria "efetivo", $\Omega_{e f} \equiv$ $\Omega_{m}-\alpha$.

Essa intrigante equivalência entre os modelos também pode ser vista mais diretamente através da equação de evolução do fator de escala. Inserindo $\mathbf{P}_{\mathbf{c}}=-\rho_{d m} \Gamma / 3 H$ na segunda equação de Friedmann, ficamos com

$$
2 a \ddot{a}+\dot{a}^{2}+\kappa-3 \alpha H_{0}^{2} a^{2}=0
$$

que deve ser comparada com

$$
2 a \ddot{a}+\dot{a}^{2}+\kappa-\Lambda a^{2}=0,
$$

que vem do modelo $\Lambda$ CDM. As equações acima implica que os modelos terão os mesmos comportamentos dinâmicos se $\alpha=\Lambda / 3 H_{0}^{2} \equiv \Omega_{\Lambda}$, que é exatamente o mesmo resultado da análise feita com o parâmetro de Hubble.

Apesar de os modelos de criação de matéria escura e $\Lambda$ CDM terem a mesma dinâmica, tais cosmologias são baseadas em hipóteses iniciais diferentes e, portanto, podem ser diferenciadas por observações atuais. Matematicamente, isso acontece por causa do papel 
desempenhado por $\alpha$. Em particular, a positividade da densidade de matéria escura (e do parâmetro de Hubble) em altos redshifts implicam que o parâmetro de criação deve satisfazer a condição $\alpha \leq \Omega_{m}$, essa condição não está presente no modelo $\Lambda$ CDM. Ainda, a dependência da contribuição de $\alpha$ com o redshift, $\alpha\left(1-(1+z)^{2}\right)$, deve modificar levemente as predições envolvendo a evolução de pequenas perturbações e o problema de formação de estruturas.

Se a planitude espacial é imposta, como predito pela inflação e sugerida por dados da radiação cósmica de fundo, temos $\Omega_{m}=1$, e

$$
\left(\frac{H}{H_{0}}\right)^{2}=(1-\alpha)(1+z)^{3}+\alpha
$$

com $\alpha$ sendo o único parâmetro livre, além de $H_{0}$, do mesmo modo que no modelo $\Lambda$ CDM plano. Agora $\Omega_{e f}=1-\alpha$.

\subsubsection{Redshift de Transição e Vínculos de Supernova}

Combinando as duas equações de Friedmann para o modelo, ficamos com

$$
\frac{\ddot{a}}{a}=-\frac{4 \pi G}{3}\left(\rho_{b a r}+\rho_{d m}+3 p_{c}\right)
$$

dado que $\mathbf{P}_{\mathbf{c}}=-\alpha \rho_{c 0}$, encontramos

$$
\frac{\ddot{a}}{a}=-\frac{4 \pi G}{3} \rho_{c 0}\left[\left(\Omega_{b a r}+\Omega_{d m}-\alpha\right)(1+z)^{3}-2 \alpha\right]
$$

Quando essa expressão se anula, pode-se achar a seguinte expressão para o redshift de transição

$$
z_{t}=\left(\frac{2 \alpha}{\Omega_{d m}-\alpha}\right)^{1 / 3}-1 .
$$

Naturalmente, para se estimar o redshift de transição é necessário vincular o valor de $\alpha$ pelas observações.

Os autores de [3] usaram os vínculos de Supernovas distantes para determinar o valor de $\alpha$. O módulo de distância de uma Supernova a um dado redshift, dado o conjunto de parâmetros $\boldsymbol{s}$, é $[19,20]$

$$
\mu_{p}(z \mid s)=m-M=5 \log d_{L}+25
$$


em que $m$ e $M$ são, respectivamente, as magnitudes aparentes e absolutas, o conjunto de parâmetros é $\boldsymbol{s} \equiv\left(H_{0}, \alpha, \Omega_{m}\right)$ e $d_{L}$ é a distância de luminosidade (em megaparsecs)

$$
d_{L}=c(1+z) \int_{0}^{z} \frac{d z^{\prime}}{H\left(z^{\prime} ; s\right)}
$$

Como podemos ver da Figura 3.4, os autores de [3], utilizando técnicas de minimização de $\chi^{2}$, obtiveram um best fit de $\alpha=0.93_{-0.26-0.44-0.63}^{+0.22+0.35+0.46}$ e $\Omega_{e f}=0.41_{-0.15-0.26-0.37}^{+0.13+0.21+0.29}$ com $68.3 \%, 95.4 \%, 99.9 \%$ de confiança estatística.

Para o caso plano, em que o único parâmetro é $\alpha$, os autores acharam $\alpha=$ $0.713_{-0.028-0.058-0.089}^{+0.027+0.052+0.077}$, mostrando que o modelo de criação de matéria escura tem um bom fit e reproduz bem os resultados do modelo $\Lambda$ CDM.

O valor do redshift de transição é implicitamente dependente da curvatura. Para um modelo curvo de criação de matéria escura, se obtém o valor central de $z_{t}=0.65$, enquanto que para o cenário plano, obtém-se $z_{t}=0.71$, valor obtido também pelo modelo $\Lambda$ CDM, ver Equação (1.83).

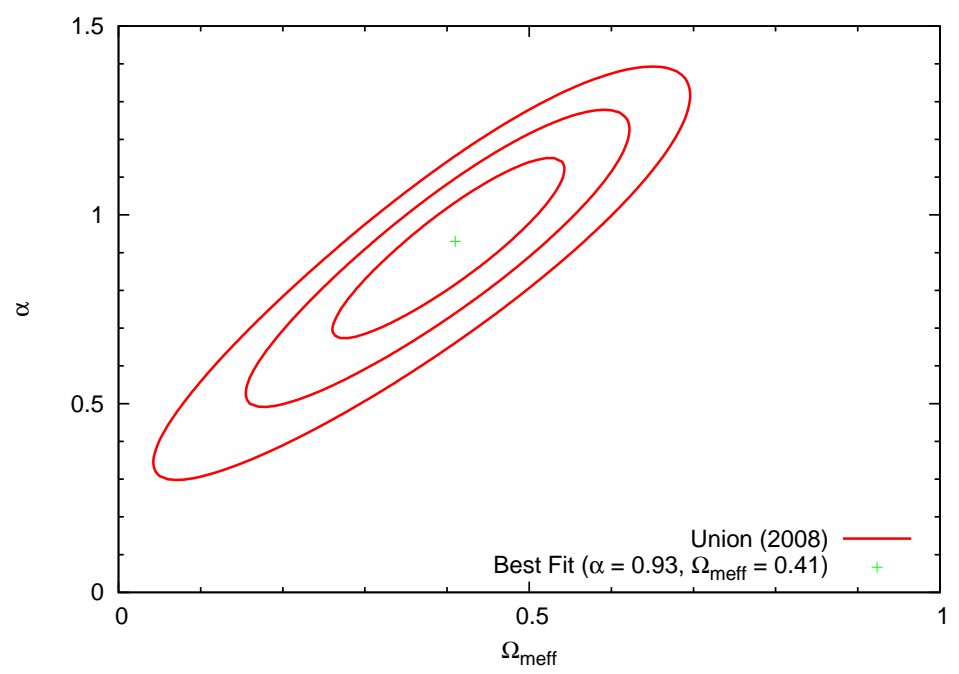

Figura 3.4: Vínculos obtidos através de análise de Supernovas do tipo Ia. Figura retirada de $[3]$.

Utilizando os recentes dados do experimento Planck [25], mais precisamente $H_{0}=$ $67.31 \pm 0.96$, podemos analisar a evolução do parâmetro de Hubble e da taxa de criação de matéria escura para o modelo LJO. Como podemos ver pela Figura (3.5), à medida que o tempo passa, $z$ vindo de $\infty$ até 0 , a taxa de criação cresce e o parâmetro de Hubble diminui.

Na Figura (3.6), podemos ver o comportamento das seguintes quantidades: $\frac{\Gamma(z)}{H(z)}, q(z)$ 


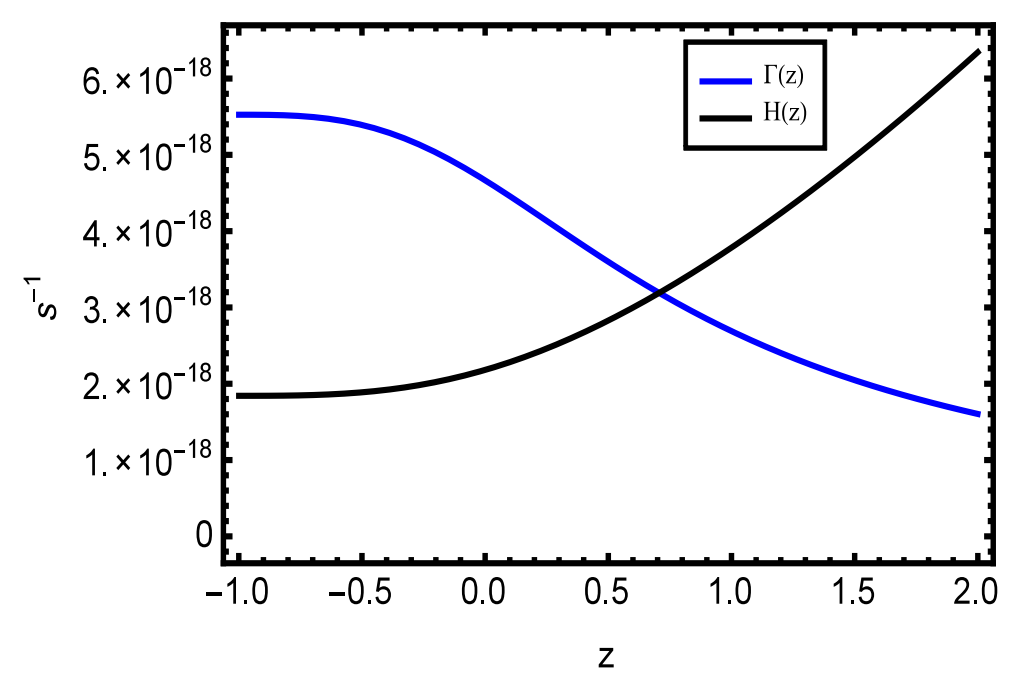

Figura 3.5: Taxa de criação de matéria escura e parâmetro de Hubble em função do redshift.

e $\frac{\mathbf{P}_{\mathbf{c}}}{\rho_{d m}}$. A linha vertical na figura mostra $z=0.71$, que é o valor do redshift discutido na seção anterior. As duas linhas horizontais correspondem aos valores 1 e $-1 / 3$. Essas linhas nos ajudarão na análise que vem a seguir.

Observando o parâmetro de desaceleração, $q(z)$ (curva preta no gráfico), vemos que para altos redshifts a expansão do Universo é desacelerada $(q(z)>0)$. O parâmetro de desaceleração começa a diminuir até atingir um valor nulo e, a partir daí, assume valores negativos $(q(z)<0)$, ou seja, a expansão do Universo se torna acelerada até hoje $(z=0)$. Seguindo o comportamento de $\frac{\Gamma(z)}{H(z)}$ (curva azul no gráfico), vemos que $q(z)$ começa a diminuir quando a taxa de criação assume valores cada vez maiores em relação à taxa de expansão do Universo. Quando a taxa de criação se torna igual à taxa de expansão do Universo, $\frac{\Gamma(z)}{H(z)}=1$, a expansão do Universo muda para o regime acelerado. Ao mesmo tempo, notamos que a pressão de criação, em comparação à densidade de matéria, $\frac{\mathbf{P}_{\mathbf{c}}}{\rho_{d m}}$, se torna cada vez mais negativa até que, em determinado momento, $\mathbf{P}_{\mathbf{c}}<-1 / 3\left(\rho_{d m}+\rho_{b a r}\right)$ (condição necessária para uma expansão acelerada pela segunda equação de Friedmann). Com auxílio da linha vertical, vemos que o instante em que todas essas condições ocorrem é quando $z=z_{t}=0.71$. Em resumo, a taxa de criação de matéria cresce até se tornar da mesma ordem (superando posteriormente) da taxa de expansão do Universo. Quando isso ocorre, a pressão de criação se torna suficientemente negativa para que a expansão seja acelerada.

Podemos atribuir valores para a taxa de criação, a fim de termos uma noção real de 


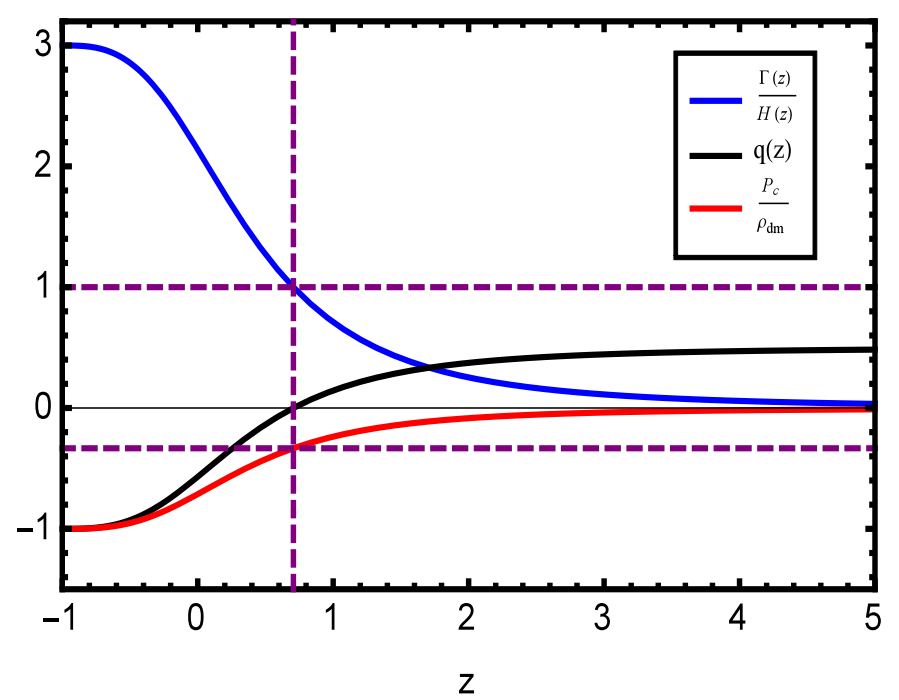

Figura 3.6: Quociente entre taxa de criação de matéria e parâmetro de Hubble, parâmetro de desaceleração e quociente entre pressão de criação e densidade de matéria criada em função do redshift.

seu funcionamento. Para isso, multiplicamos a taxa de criação pela densidade de matéria criada. Com isso, temos quanta matéria é criada por unidade de tempo e volume. A Figura (3.7) mostra o comportamento dessa quantidade. Para hoje, $z=0$, essa taxa seria de, aproximadamente, $1.6 \times 10^{12}$ massas solares por volume de Hubble por ano.

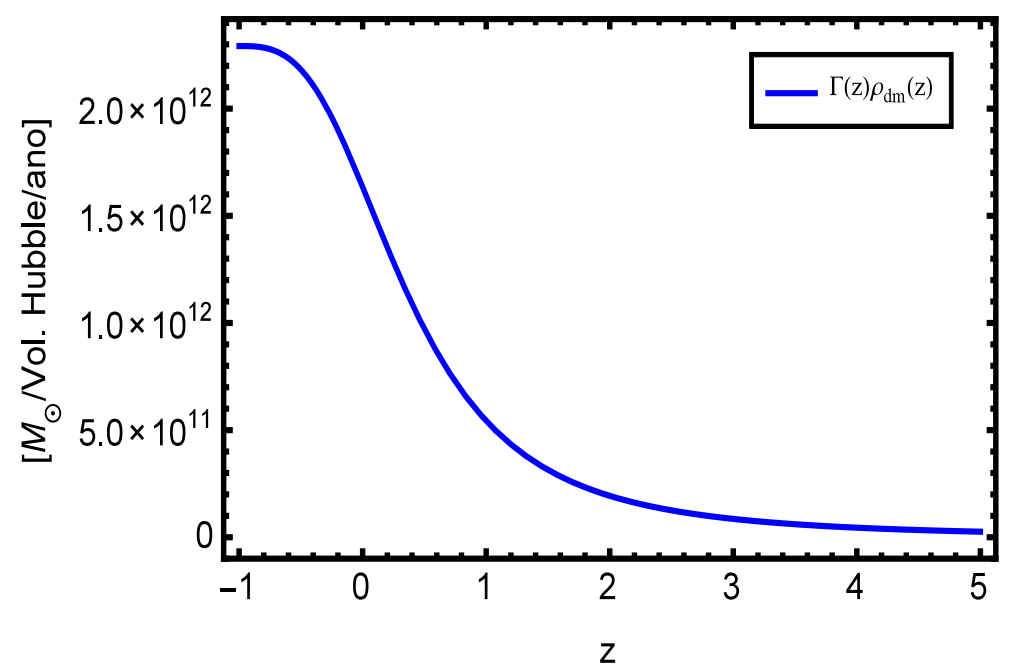

Figura 3.7: Quantidade de matéria criada por unidade de volume de Hubble por ano em função do redshift.

Além dos estudos em nível de background feitos por [3] (como visto na seção anterior, o modelo LJO possui a mesma evolução para o parâmetro de Hubble que o modelo $\Lambda$ CDM), o modelo LJO foi testado em nível perturbativo a fim de quebrar a degenerescência com $\Lambda$ CDM. Primeiramente, foram utilizadas as equações neo-Newtonianas para determinar a 
evolução das perturbações lineares [69]. Posteriormente, de forma mais detalhada, utilizando a abordagem neo-Newtoniana relativística para perturbações lineares e relativística para perturbações não-lineares $[70,71]$. A conclusão a que os autores dos trabalhos acima citados chegaram é que em todos os níveis os modelos são degenerados. Mais recentemente, assumindo que todas componentes são criadas (e não somente matéria), obteve-se a mesma degenerescência [72]. 


\section{Capítulo 4}

\section{Criação Gravitacional de Partículas: Formulação Cinética}

As propriedades termodinâmicas do modelo de criação de matéria escura foram estudados na Seção 3.1. Buscamos agora uma justificativa cinética. Para isso, proporemos um termo de criação para o lado direito da equação de Boltzmann. A primeira tentativa de modificação da equação de Boltzmann nesse contexto foi proposta por [73]. Os resultados que serão mostrados nesse capítulo estão disponíveis em $[17,18]$.

A forma mais geral para a equação de Boltzmann é

$$
\mathcal{L}[f]=\mathcal{C}[f]+\mathcal{P}_{g}[f]
$$

em que $\mathcal{L}$ é o operador de Liouville, $\mathcal{C}$ é o termo colisional e $\mathcal{P}_{g}$ é o termo responsável pela produção de partículas a partir do campo gravitacional. Para seguir a análise, tomaremos $\mathcal{C}=0$. Essa escolha se justifica pelos seguintes motivos: as consequências do termo colisional já foram intensivamente investigadas na literatura e, talvez, mais importante: a natureza do termo $\mathcal{P}_{g}$ é intrinsecamente diferente das contribuições colisionais usuais, precisando de um tratamento alternativo.

Modelaremos o termo de criação tendo em mente que as partículas são criadas a partir do campo gravitacional e, portanto, o novo termo deve levar em conta a gravidade. No contexto da Relatividade Geral, isso quer dizer que o efeito desse termo deve desaparecer numa métrica de Minkowski. Tal condição pode ser garantida supondo que o termo de criação é proporcional aos símbolos de Christoffel e, portanto, se reduz a zero quando a 
métrica é minkowskiana. Também devemos levar em consideração que o processo deve depender da razão (ver Equação (3.24))

$$
\frac{\Gamma}{\Theta},
$$

em que $\Gamma$ é a taxa de criação de partículas e $\Theta=3 H$ é a taxa de expansão do Universo. Com essas duas condições, temos que

$$
\mathcal{P}_{g}=-\frac{\Gamma}{\Theta} \Gamma_{\alpha \beta}^{\mu} P^{\alpha} P^{\beta} L_{\mu}
$$

em que $L_{\mu}$ é um vetor que será determinado. Dessa maneira garantimos que o termo de criação se anula quando a taxa de produção é zero e quando o campo gravitacional é desprezível, podendo assim ser considerada a métrica de Minkowski, que possui conexões nulas.

Assumiremos que o vetor $L_{\mu}$ é uma combinação linear de $P_{\mu}$ e a primeira derivada de $f$, tomando a forma

$$
L_{\mu}=A \frac{\partial f}{\partial P^{\mu}}+B P_{\mu}
$$

então, a equação de Boltzmann (2.70) pode ser escrita como

$$
P^{\mu} \frac{\partial f}{\partial x^{\mu}}-\Gamma_{\alpha \beta}^{\mu} P^{\alpha} P^{\beta} \frac{\partial f}{\partial P^{\mu}}=-\frac{\Gamma}{\Theta} \Gamma_{\alpha \beta}^{\mu} P^{\alpha} P^{\beta}\left(A \frac{\partial f}{\partial P^{\mu}}+B P_{\mu}\right) .
$$

Portanto, como visto ao final do capítulo anterior, a equação de Boltzmann com termo colisional nulo na camada de massa tem a seguinte forma:

$$
P^{\mu} \frac{\partial f}{\partial x^{\mu}}-\Gamma_{\alpha \beta}^{i} P^{\alpha} P^{\beta} \frac{\partial f}{\partial P^{i}}=0
$$

mostrando que a componente 0 não aparece no segundo termo. Seguindo esse raciocínio, impomos que $L_{0}=0$ e obtemos $B=-\frac{A}{P_{0}} \frac{\partial f}{\partial P^{0}}$. Assim, a forma final da equação de Boltzmann é

$$
P^{\mu} \frac{\partial f}{\partial x^{\mu}}-\Gamma_{\alpha \beta}^{\mu} P^{\alpha} P^{\beta} \frac{\partial f}{\partial P^{\mu}}=-A \frac{\Gamma}{\Theta} \Gamma_{\alpha \beta}^{\mu} P^{\alpha} P^{\beta}\left(\frac{\partial f}{\partial P^{\mu}}-\frac{P_{\mu}}{P_{0}} \frac{\partial f}{\partial P^{0}}\right) .
$$

Vamos agora impor que a condição de que os quadri-momentos admissíveis estão dentro da camada de massa, isto é, $P^{\mu} P_{\mu}=m^{2}$. Isso implica 


$$
P^{\mu} \frac{\partial f}{\partial x^{\mu}}-\Gamma_{\alpha \beta}^{i} P^{\alpha} P^{\beta} \frac{\partial f}{\partial P^{i}}=-A \frac{\Gamma}{\Theta} \Gamma^{i}{ }_{\alpha \beta} P^{\alpha} P^{\beta} \frac{\partial f}{\partial P^{i}},
$$

em que $A$ é um número que será fixado a fim de reproduzir exatamente a abordagem termodinâmica do modelo com criação de partículas.

Substituindo os símbolos de Christoffel da métrica de FLRW, ficamos com

$$
P^{0} \frac{\partial f}{\partial t}-2 H P^{0} P^{i} \frac{\partial f}{\partial P^{i}}=-2 A \frac{\Gamma}{\Theta} H P^{0} P^{i} \frac{\partial f}{\partial P^{i}}
$$

como feito anteriormente, passaremos a equação acima, que está escrita em termos dos momentos comóveis, para os momentos físicos, $p^{i}=a P^{i}$. Para isso, utilizaremos a seguinte relação entre as derivadas temporais de $f$ :

$$
\begin{aligned}
\frac{\partial f\left(t, P^{i}\right)}{\partial t} & =\frac{\partial f\left(t, p^{i}\right)}{\partial t}+\frac{\partial p^{i}}{\partial t} \frac{\partial f\left(t, p^{i}\right)}{\partial p^{i}} \\
& =\frac{\partial f\left(t, p^{i}\right)}{\partial t}+\frac{\dot{a}}{a} p^{i} \frac{\partial f\left(t, p^{i}\right)}{\partial p^{i}}
\end{aligned}
$$

em que foi usado $\frac{\partial p^{i}}{\partial t}=\dot{a} P^{i}=\frac{\dot{a}}{a} p^{i}$. Portanto, a equação escrita em momentos físicos fica

$$
p^{0} \frac{\partial f}{\partial t}-H p^{0} p^{i} \frac{\partial f}{\partial p^{i}}=-2 A \frac{\Gamma}{\Theta} H p^{0} p^{i} \frac{\partial f}{\partial p^{i}},
$$

e, como será visto adiante, a escolha apropriada para a constante arbitrária é $A=\frac{1}{2}$. Com isso, a equação de Boltzmann para o modelo de criação de partículas assume a forma final

$$
E \frac{\partial f}{\partial t}-\frac{\dot{a}}{a}\left(1-\frac{\Gamma}{\Theta}\right) E p \frac{\partial f}{\partial p}=0
$$

em que $p^{i}$ foi substituído por $p$ devido à isotropia do espaço.

Vale notar que a forma do termo de produção gravitacional proposto aqui tem uma concepção completamente diferente da apresentada em [73]. Lá, uma separação de $\mathcal{P}_{g}$ ( $H$ na notação dos autores), de alguma forma inspirada no termo colisional usual, foi proposta (ver equação (37) em [73]). 


\subsection{Equações de Balanço}

Usaremos as definições de quantidades macroscópicas de fluxo de partículas, fluxo de entropia e do tensor de energia-momento

$$
\begin{array}{r}
N^{\mu}=\int f P^{\mu} a^{3} \frac{d^{3} P}{P^{0}}, \\
S^{\mu}=-\int(f \ln f-f) P^{\mu} a^{3} \frac{d^{3} P}{P^{0}}, \\
T_{\text {coll }}^{\mu \nu}=\int f P^{\mu} P^{\nu} a^{3} \frac{d^{3} P}{P^{0}},
\end{array}
$$

junto com (4.12) para recuperar os resultados de 3.1. Observando que, devido à isotropia do espaço, as únicas componentes não nulas de $N^{\mu}$ e $S^{\mu}$ são

$$
\begin{array}{r}
N^{0}=n=\int f d^{3} p, \\
S^{0}=s=-\int(f \ln f-f) d^{3} p,
\end{array}
$$

enquanto que o tensor de energia-momento tem uma forma diagonal tal que a densidade de energia é dada por

$$
\rho=u_{\mu} u_{\nu} T^{\mu \nu}=T^{00}
$$

Isso significa dizer que qualquer correção isotrópica $\Delta T^{\mu \nu}$ aos estados de equilíbrio tem $\Delta T^{00}=0$.

Calcularemos agora a divergência dos vetores e tensores definidos no começo dessa seção.

- Fluxo de partículas:

$$
\begin{aligned}
N^{\mu}{ }_{\mu} & \equiv \frac{1}{a^{3}} \frac{\partial}{\partial x^{\mu}}\left(a^{3} \int f P^{\mu} a^{3} \frac{d^{3} P}{P^{0}}\right)=\frac{1}{a^{3}} \frac{\partial}{\partial t}\left(a^{3} \int f d^{3} p\right) \\
& =3 H n+H\left(1-\frac{\Gamma}{\Theta}\right) \int p \frac{\partial f}{\partial p} d^{3} p \\
& =n \Gamma,
\end{aligned}
$$

que está de acordo com (3.7), com $\Psi=n \Gamma$.

- Fluxo de entropia: 


$$
\begin{aligned}
S_{{ }_{; \mu}} & \equiv-\frac{1}{a^{3}} \frac{\partial}{\partial x^{\mu}}\left(a^{3} \int(f \ln f-f) P^{\mu} a^{3} \frac{d^{3} P}{P^{0}}\right) \\
& =-\frac{1}{a^{3}} \frac{\partial}{\partial t}\left(a^{3} \int(f \ln f-f) d^{3} p\right) \\
& =3 s H-\int \frac{\partial f}{\partial t} \ln f d^{3} p \\
& =3 s H-H\left(1-\frac{\Gamma}{\Theta}\right) \int p \frac{\partial f}{\partial p} \ln f d^{3} p \\
& =-\Gamma \int(f \ln f-f) d^{3} p \\
& =s \Gamma,
\end{aligned}
$$

que é o mesmo resultado que (3.15) para $\dot{\sigma}=0$ e $\Psi=n \Gamma$.

- Tensor energia-momento:

Vamos agora calcular o divergente do tensor de energia-momento total projetado na direção da quadrivelocidade $u_{\mu}$. Isso envolve um aspecto sutil e delicado do ponto de vista cinético. De fato, as equações de campo de Einstein necessitam um tensor de energia-momento de divergência nula e sabemos que a pressão de criação é negativa (para um Universo em expansão) e de origem não-colisional. Contudo, lembramos que, diferentemente da densidade de energia, não temos restrição na pressão cinética para estados fora do equilíbrio [55]. Dessa maneira, podemos assumir aqui (assim como na abordagem macroscópica) a existência de um termo de correção não-colisional para a pressão. Agora, vamos supor também que essa correção toma a forma $\Delta \tilde{T}_{j}^{i}=-\tilde{\mathbf{P}} \delta_{j}^{i}$ ou, equivalentemente, $\Delta \tilde{T}^{\mu \nu}=-\tilde{\mathbf{P}} h^{\mu \nu}(\tilde{\mathbf{P}}$ é uma pressão desconhecida e não necessariamente igual ao valor de equilíbrio local macroscópico). Nesse caso, podemos escrever

$$
u_{\mu} T_{; \nu}^{\mu \nu} \equiv u_{\mu}\left[\frac{1}{a^{3}} \frac{\partial}{\partial x^{\nu}}\left(a^{3} T^{\mu \nu}\right)+\Gamma_{\alpha \beta}^{\mu} T^{\alpha \beta}\right]
$$


com $T^{\mu \nu}=T_{\text {coll }}^{\mu \nu}+\Delta \tilde{T}^{\mu \nu}$, em que $T_{\text {coll }}^{\mu \nu}$ é dada pela Eq. (4.15). Ficando assim:

$$
\begin{aligned}
u_{\mu} T^{\mu \nu}{ }_{\nu} & \equiv \frac{1}{a^{3}} \frac{\partial}{\partial t}\left(a^{3} T_{\text {coll }}^{00}\right)+\Gamma_{i j}^{0}\left(T_{\text {coll }}^{i j}+\Delta \tilde{T}^{i j}\right) \\
& =\frac{1}{a^{3}} \frac{\partial}{\partial t}\left(a^{3} \int f E d^{3} p\right)+3 \frac{\dot{a}}{a}(\mathbf{P}+\tilde{\mathbf{P}}) \\
& =3 H(\rho+\mathbf{P}+\tilde{\mathbf{P}})+H\left(1-\frac{\Gamma}{\Theta}\right) \int E p \frac{\partial f}{\partial p} d^{3} p \\
& =3 H(\rho+\mathbf{P}+\tilde{\mathbf{P}})-3 H\left(1-\frac{\Gamma}{\Theta}\right)(\rho+\mathbf{P}) \\
& =3 H \tilde{\mathbf{P}}+(\rho+\mathbf{P}) \Gamma .
\end{aligned}
$$

Portanto, obtemos um tensor energia-momento total de divergência nula, tal como exigido pelas equações de campo de Einstein, somente se a pressão de criação $\tilde{\mathbf{P}}$ for dada por

$$
\tilde{\mathbf{P}}=-(\rho+\mathbf{P}) \frac{\Gamma}{\Theta} \equiv \mathbf{P}_{\mathbf{c}}
$$

em completo acordo com a expressão macroscópica (veja Eq.(3.17)).

É importante frisar que quando não há criação de partículas, todos os resultados de equilíbrio são recuperados. Ou seja, quando $\Gamma=0$, então $N^{\mu}{ }_{\mu}=0, S^{\mu} ;_{\mu}=0$ e $u_{\alpha} T^{\alpha \beta} ;_{\beta}=0$, como esperado.

\subsection{Lei de Temperatura}

Vamos agora determinar a lei de evolução da temperatura para fluidos descritos por esse processo. Inserindo a função distribuição $f=e^{\alpha-\beta E}$ na equação (4.12), obtemos

$$
\dot{\alpha}-\dot{\beta} E+H \beta\left(1-\frac{\Gamma}{\Theta}\right) \frac{p^{2}}{E}=0 .
$$

Essa equação tem dois limites. O limite relativístico $(m=0, E=p)$ e o limite não relativístico $\left(m \gg T, E=m+\frac{p^{2}}{2 m}\right)$. Os dois casos serão investigados a seguir.

- $m=0$

Nesse caso, a expressão acima fica

$$
\frac{\dot{\alpha}}{\dot{\beta}}=E\left[1-\left(1-\frac{\Gamma}{\Theta}\right) \frac{\dot{a}}{a} \frac{\beta}{\dot{\beta}}\right],
$$


que possui solução $\dot{\alpha}=0 \operatorname{com} \beta=1 / T$, recuperando o resultado da termodinâmica de não equilíbrio, veja (3.20)

$$
\frac{\dot{T}}{T}=-\frac{\dot{a}}{a}+\frac{\Gamma}{3} .
$$

A solução geral da equação acima pode ser escrita como

$$
T=T_{0}\left(\frac{a_{0}}{a}\right) e^{-\frac{1}{3} \int_{t}^{t_{0}} \Gamma\left(t^{\prime}\right) d t^{\prime}} .
$$

Em termos do redshift, a solução geral $T(z)$ para um fluido CMB dotado de uma criação "adiabática" de partículas é dada por

$$
T=T_{0}(1+z) e^{\frac{1}{3} \int_{0}^{z} \Gamma\left(z^{\prime}\right) \frac{d t}{d z^{\prime}} d z^{\prime}} .
$$

- $m \gg T$

Nesse limite, a equação se torna

$$
\frac{\dot{\alpha}}{\dot{\beta}}-m=\frac{p^{2}}{m}\left[\frac{1}{2}-H \frac{\beta}{\dot{\beta}}\left(1-\frac{\Gamma}{\Theta}\right)\right],
$$

que possui solução $\alpha-m \beta=$ const $\mathrm{e}$

$$
\frac{\dot{T}}{T}=-2\left(\frac{\dot{a}}{a}-\frac{\Gamma}{3}\right) .
$$

A solução geral $T(z)$ para um fluido não relativístico dotado de uma criação "adiabática" de partículas é

$$
T=T_{0}(1+z)^{2} e^{\frac{1}{3} \int_{0}^{z} \Gamma\left(z^{\prime}\right) \frac{d t}{d z^{\prime}} d z^{\prime}} .
$$

Como uma ilustração do formalismo desenvolvido, podemos considerar a lei fenomenológica $\Gamma=3 \beta H$, discutida na literatura $[61,74]$. Podemos ver que a solução para a temperatura em termos do redshift é

$$
T=T_{0}(1+z)^{1-\beta}
$$


para o caso relativístico e

$$
T=T_{0}(1+z)^{2(1-\beta)}
$$

para o não relativístico. Naturalmente, quando $\beta=0$ (sem produção), os resultados usuais são recuperados.

Para o modelo LJO, $\Gamma=3 \alpha\left(\frac{\rho_{c 0}}{\rho_{d m}}\right) H$, a evolução de temperatura para um fluido relativístico fica

$$
\frac{\dot{T}}{T}=-\frac{\dot{a}}{a}\left(1-\alpha \frac{H_{0}^{2}}{H^{2}}\right)
$$

Substituindo $\frac{H^{2}}{H_{0}^{2}}=\frac{1-\alpha}{a^{3}}+\alpha$ na expressão acima, temos a solução $T(a)$

$$
T(a)=T_{0} \frac{\left[1+\alpha\left(a^{3}-1\right)\right]^{1 / 3}}{a}
$$

Já para o caso não-relativístico, a equação de evolução é

$$
\frac{\dot{T}}{T}=-2 \frac{\dot{a}}{a}\left(1-\alpha \frac{H_{0}^{2}}{H^{2}}\right),
$$

cuja solução é dada por:

$$
T(a)=T_{0} \frac{\left[1+\alpha\left(a^{3}-1\right)\right]^{2 / 3}}{a^{2}} .
$$

Assim como no caso anterior, quando não há criação de matéria, $\alpha=0$, os resultados usuais são reobtidos. 


\section{Capítulo 5}

\section{Nova Equação de Relíquias Cósmicas}

Vamos agora investigar como a criação gravitacional de partículas, representada pela equação de Boltzmann generalizada (4.12), modifica a equação de evolução de relíquias cósmicas $\chi$.

Para estudar esse processo, é necessário recolocar o termo colisional $\mathcal{C}[f]=\mathcal{C}_{e}[f]+\mathcal{C}_{i}[f]$, em que "e" representa processos elásticos e "i" processos inelásticos [55], na equação de Boltzmann

$$
\mathcal{L}[f]=\mathcal{C}[f]
$$

em que

$$
\mathcal{L}(f) \equiv E\left[\frac{\partial f}{\partial t}-H\left(1-\frac{\Gamma}{\Theta}\right) p \frac{\partial f}{\partial p}\right]
$$

é o operador de Liouville generalizado.

Multiplicando por $\frac{g_{\chi}}{(2 \pi)^{3}} d^{3} p$ e integrando, obtém-se

$$
\frac{d n_{\chi}}{d t}+\left(3 \frac{\dot{a}}{a}-\Gamma_{\chi}\right) n_{\chi}=\frac{g_{\chi}}{(2 \pi)^{3}} \int \mathcal{C}[f] \frac{d^{3} p}{E}
$$

em que

$$
n_{\chi}=\frac{g_{\chi}}{(2 \pi)^{3}} \int f d^{3} p
$$

é a densidade de número de partículas e $g_{\chi}$ são os graus de liberdade da relíquia e $\Gamma_{\chi}$ é a taxa de produção gravitacional da relíquia.

No Apêndice B obtemos o lado direito da Equação (5.3). Com esse resultado, podemos 


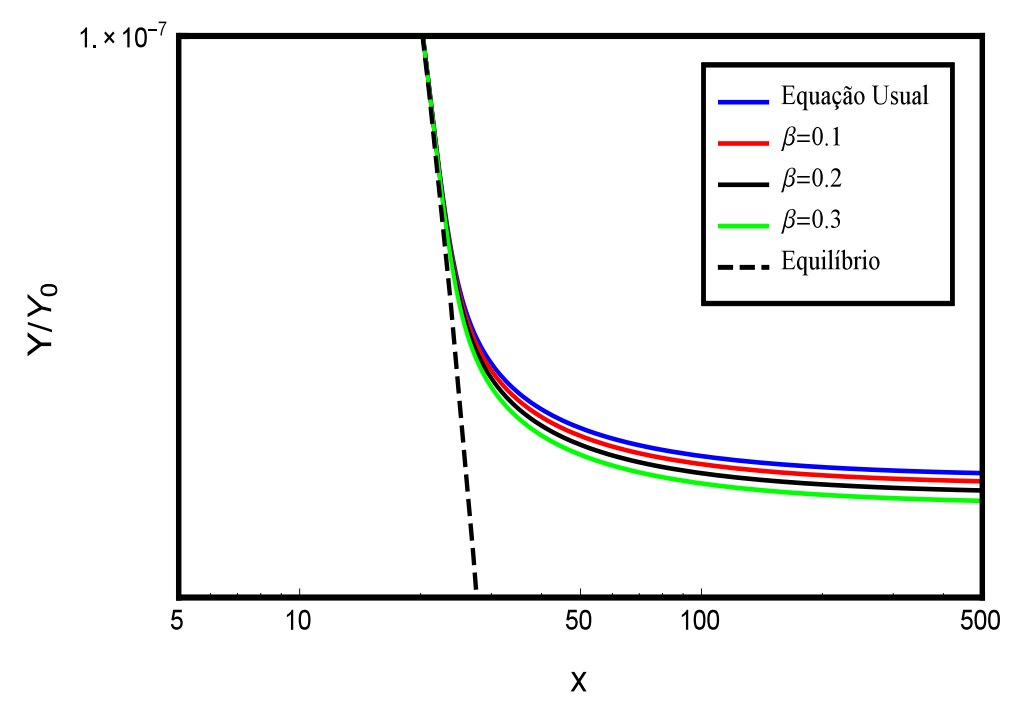

Figura 5.1: Soluções da nova equação de evolução para diferentes valores de $\beta$. O parâmetro $\lambda$ foi fixado em $\lambda=10^{13}$. Mais detalhes no texto.

escrever a equação de evolução da seguinte maneira:

$$
\frac{d n_{\chi}}{d t}+\left(3 \frac{\dot{a}}{a}-\Gamma_{\chi}\right) n_{\chi}=-<\sigma v>\left(n_{\chi}^{2}-n_{\chi e q}^{2}\right)
$$

em que $\langle\sigma v\rangle$ é a média térmica da taxa de aniquilação . Essa equação generaliza a conhecida forma na ausência de processos gravitacionais de criação de partículas.

Vemos que a produção gravitacional de partículas, quantificada por $\Gamma_{\chi}$, aparece como um desvio da equação de evolução usual. O processo físico descrito pela equação acima é o seguinte. Para $T \gg m_{\chi}, \chi$ se comporta como radiação e sua densidade de equilíbrio é $n_{\chi e q} \propto T^{3}$. Quando o Universo esfria, eventualmente $T \ll m_{\chi}$ e a densidade de equilíbrio de $\chi$ passa a ser $n_{\chi e q} \propto T^{3 / 2} e^{-m_{\chi} / T}$. Durante a evolução do Universo, $n_{\chi}$ segue $n_{\chi e q}$ até que sua densidade se torna muito pequena e as partículas $\chi$ não conseguem se aniquilar e o lado direito da equação (5.5) não tem mais efeito sobre a equação de evolução. Esse é o chamado freeze-out: as partículas param de se aniquilar e perdem contato com sua densidade de equilíbrio, $n_{\chi e q}$. Se as partículas $\chi$ ficassem sempre em equilíbrio, sua abundância decairia exponencialmente até que não houvesse mais relíquias mensuráveis. Veja Figura (5.1).

Usando a definição $Y=\frac{n_{\chi}}{s}$, em que $s$ é a densidade de entropia e, nesse caso considerado (produção "adiabática" de partículas, $\dot{\sigma}=0)[59], \dot{s}=-3 H s\left(1-\frac{\Gamma_{\gamma}}{3 H}\right)$, em que $\Gamma_{\gamma}$ é a taxa de produção de fótons (como no caso usual, a densidade de entropia é governada 
pela radiação), se obtém

$$
\frac{d Y}{d t}+\left(\Gamma_{\gamma}-\Gamma_{\chi}\right) Y=-s<\sigma v>\left(Y^{2}-Y_{e q}^{2}\right)
$$

A equação acima pode ser reescrita da seguinte forma:

$$
\frac{d Y}{d x}+\frac{\Gamma_{\gamma}-\Gamma_{\chi}}{H x\left(1-\frac{\Gamma_{\gamma}}{3 H}\right)} Y=-\frac{s<\sigma v>}{H x\left(1-\frac{\Gamma_{\gamma}}{3 H}\right)}\left(Y^{2}-Y_{e q}^{2}\right)
$$

em que $x=\frac{m_{\chi}}{T}$ e foi usado $\frac{d Y}{d t}=-x \frac{d Y}{d x} \frac{\dot{T}}{T}=x\left(1-\frac{\Gamma_{\gamma}}{3 H}\right) H \frac{d Y}{d x}$. Além disso, consideramos a lei de temperatura para o banho relativístico (4.26):

$$
\frac{\dot{T}}{T}=-\frac{\dot{a}}{a}+\frac{\Gamma_{\gamma}}{3}
$$

A Equação (5.7) é a equação de evolução de relíquias mais geral quando partículas e fótons são criados pelo campo gravitacional. Analisaremos o problema particular quando as taxas de criação dos fótons e relíquias são os mesmos ou da mesma ordem de grandeza, $\Gamma_{\gamma} \approx \Gamma_{\chi}$. Isso leva a uma simplificação do segundo termo do lado esquerdo.

O processo ocorre durante a era da radiação, em que $s=\frac{2 \pi^{2}}{45} g_{\star, S} T^{3}, \rho=\frac{\pi^{2}}{30} g_{\star} T^{4}$ e $H^{2}=\frac{8 \pi G}{3} \rho . g_{\star, S}$ e $g_{\star}$ contam diferentes graus de liberdade relativísticos para a densidade de entropia e densidade de energia, respectivamente. Substituindo essas quantidades na equação acima, obtemos

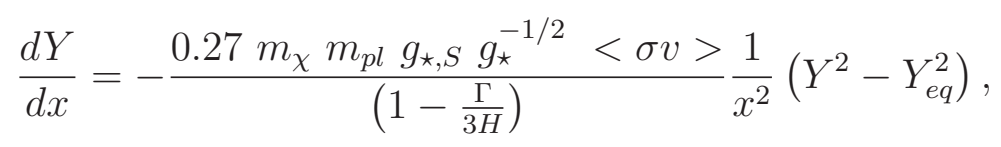

em que $m_{\chi}$ é a massa da relíquia e $m_{p l}=G^{-1 / 2}$ é massa de Planck. Com base no trabalho de Drees, Iminniyaz e Kakizaki [75], fixaremos $g=g_{\star, S}=g_{\star}=90$. A partícula a ser considerada aqui será um férmion com $g_{\chi}=2$ e $m_{\chi}=100 \mathrm{GeV}$. Naturalmente, quando $\Gamma=0$, a equação de evolução usual é recuperada (2.101).

\subsection{Caso $\Gamma=3 \beta H$}

Como exemplo, consideraremos um processo com taxa de criação de matéria $\Gamma_{\chi}=\Gamma_{\gamma}=$ $\Gamma=3 \beta H$. Para esse modelo, a densidade de partículas das relíquias e densidade de 
entropia para estágios finais são

$$
n_{\chi} \propto a^{-3(1-\beta)}, s \propto a^{-3(1-\beta)} .
$$

Portanto, é esperado que $Y$ evolua para um valor constante em estágios finais, condição que demonstraremos a seguir.

A equação de evolução é

$$
\frac{d Y}{d x}=-\frac{\lambda}{(1-\beta)} \frac{1}{x^{2}}\left(Y^{2}-Y_{e q}^{2}\right)
$$

em que $\lambda=0.27 m_{\chi} m_{p l} \sqrt{g}<\sigma v>$.

Como mencionado por [76], $\lambda$ tem valores na ordem de $10^{8}-10^{14}$ e para esse intervalo a equação acima é numericamente dura ("stiff"). Usando a troca de variável proposta por eles, $W=\ln Y$, ficamos com

$$
\frac{d W}{d x}=-\frac{\lambda}{(1-\beta)} \frac{1}{x^{2}}\left[e^{W}-e^{\left(2 W_{e q}-W\right)}\right]
$$

Primeiro, resolvemos a equação de $x=1$ a $x=500$ para ver como diferentes valores de $\beta$ afetam a evolução da equação. A Figura (5.1) mostra a diferença entre a equação usual e a nova equação para diferentes valores de $\beta$. Como se pode ver na figura, a presença de um processo constante de criação de matéria faz com que a relíquia desacople mais tarde e com uma abundância menor do que no caso usual.

Agora, plotaremos a diferença percentual $\Delta Y=\frac{Y-Y_{\text {usual }}}{Y_{\text {usual }}}$, em que $Y_{\text {usual }}$ é a equação usual. Como se pode ver pela Figura (5.2), a abundância da relíquia pode mudar por fatores da ordem de $10 \%$ a $30 \%$, dependendo do valor de $\beta$.

Podemos buscar por soluções semi-analíticas para extrairmos parâmetros cosmológicos $[21,22,75]$. No Universo jovem, $x \ll x_{f}$, em que $x_{f}$ é o $x$ no freeze-out, a solução exata da equação de evolução segue a solução de equilíbrio. A equação de evolução em termos de $\Delta$ é

$$
\frac{d \Delta}{d x}=-\frac{d Y_{e q}}{d x}-\frac{\lambda}{(1-\beta)} \frac{\Delta}{x^{2}}\left(2 Y_{e q}+\Delta\right)
$$

que tem a seguinte solução para $x \gg 1$

$$
\Delta=\frac{(1-\beta) x^{2}}{2 \lambda}
$$




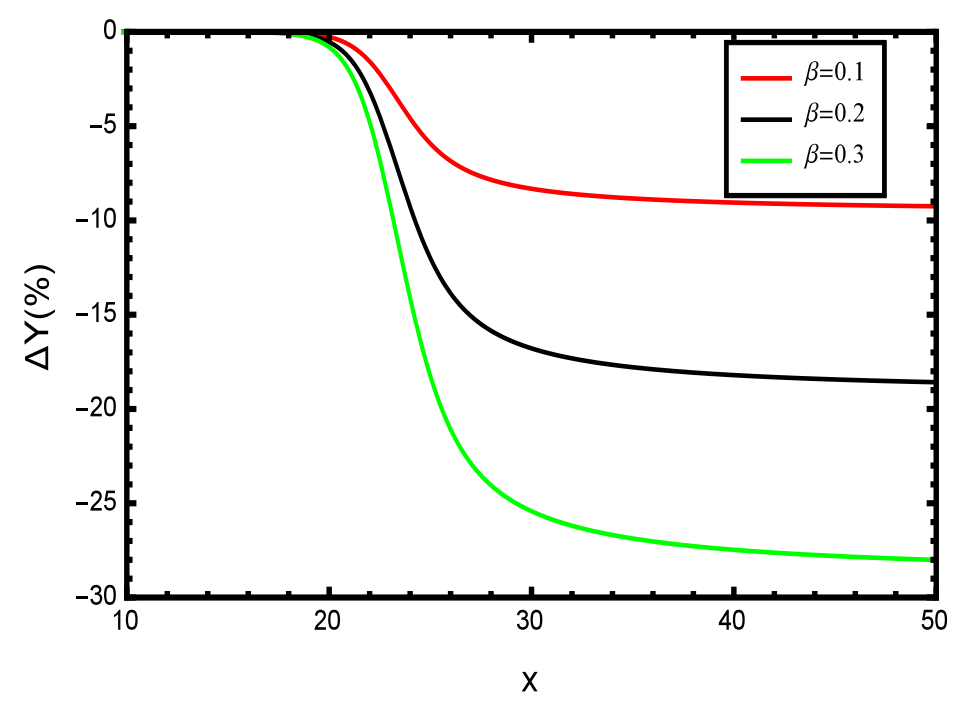

Figura 5.2: Diferença percentual $\Delta Y=\frac{Y-Y_{\text {usual }}}{Y_{\text {usual }}}$ para diferentes valores de $\beta, \lambda=10^{13}$.

e foi considerado que $\frac{d Y_{e q}}{d x} \approx-Y_{e q}$ nesse regime.

O freeze-out ocorre quando $\Delta \equiv Y-Y_{e q}=\varepsilon Y_{e q}$, em que $\varepsilon$ é da ordem de 1 . Colocando esse resultado na condição de freeze-out, obtemos uma equação para $x_{f}$

$$
x_{f}=\ln \frac{0.0017 \varepsilon m_{\chi} m_{p l} \sqrt{g}<\sigma v>}{(1-\beta) x_{f}} .
$$

Usando $\varepsilon=\sqrt{2}-1$ [75] e valores típicos para a $\langle\sigma v>$ de WIMPs, temos que o freeze-out ocorre em $x_{f} \approx 21-22$, dependendo do valor de $\beta$.

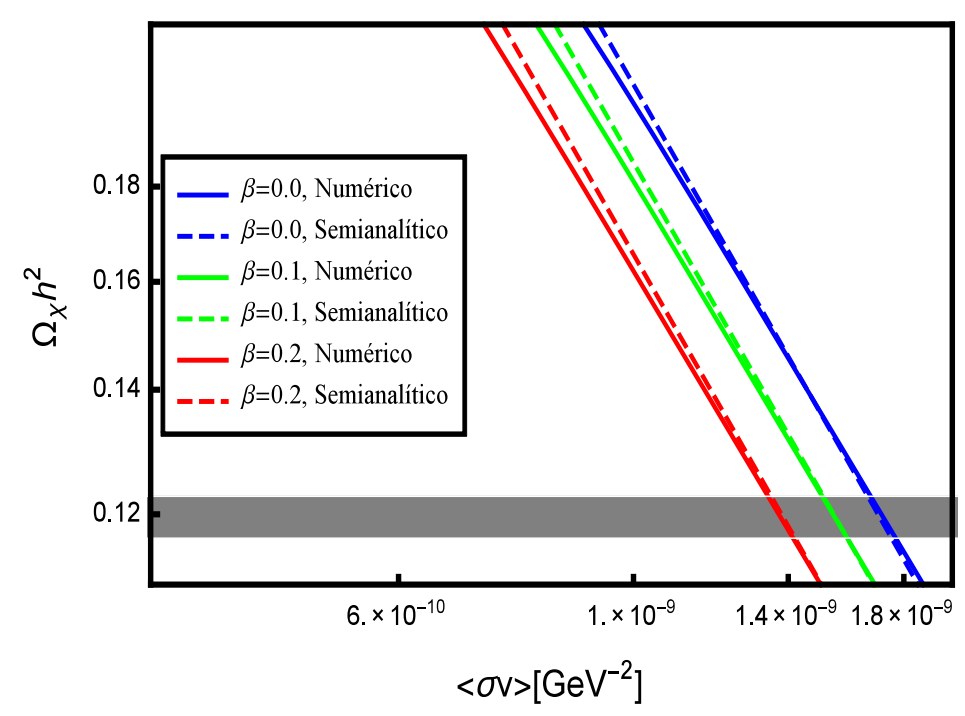

Figura 5.3: Parâmetro de densidade de relíquias $\Omega_{\chi} h^{2}$ como função de $\langle\sigma v\rangle$.

Para épocas muito posteriores ao freeze-out $\left(x \gg x_{f}\right)$, a eficiência da produção de relíquias pelo banho térmico se torna desprezível, isso significa que o termo $\left\langle\sigma v>Y_{c}^{2}\right.$ 


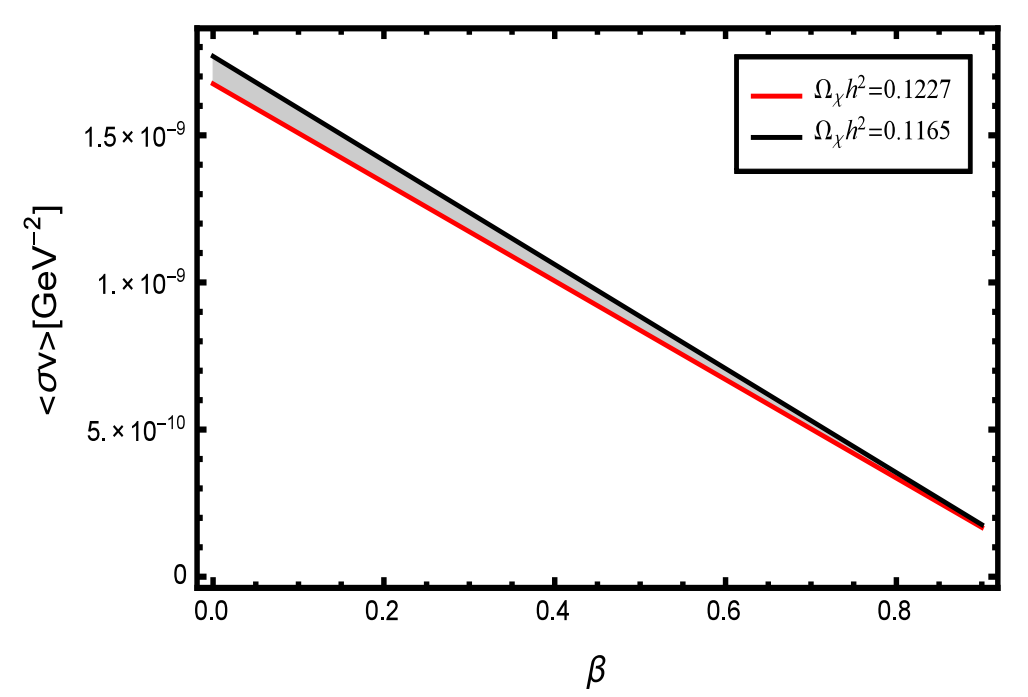

Figura 5.4: $\left\langle\sigma v>\right.$ como função de $\beta$ para o best-fit do experimento Planck $\Omega_{\chi} h^{2}=$ $0.1196 \pm \sigma$.

pode ser ignorado e que a equação a ser resolvida é

$$
\frac{d Y}{d x}=\frac{-\lambda}{(1-\beta)} \frac{Y^{2}}{x^{2}}
$$

que possui a solução

$$
Y(x \rightarrow \infty)=\frac{(1-\beta)}{\lambda} x_{f}
$$

Lembrando que a densidade do WIMP hoje é $\rho_{\chi, 0}=m_{\chi} n_{\chi, 0}=m_{\chi} s_{0} Y(\infty)$ e usando $s_{0}=2900 \mathrm{~cm}^{-3}$, podem-se verificar os efeitos de $\beta$ sobre $\Omega_{\chi} h^{2}$ como função de $\langle\sigma v>$. Mais precisamente,

$$
\Omega_{\chi} h^{2}=\frac{\rho_{\chi, 0}}{\rho_{c, 0}} h^{2}=(1-\beta) 8.5 \times 10^{-11} \frac{x_{f}}{\sqrt{g}<\sigma v>} .
$$

Na Figura (5.3), ao assumirmos $m_{\chi}=100 \mathrm{GeV}$, mostramos o comportamento de $\Omega_{\chi} h^{2}$ como função da seção de choque para diferentes valores de $\beta$, como descrito na figura. A região em cinza demarca os vínculos $\Omega_{\chi} h^{2}=0.1196 \pm 0.0031$ ( $1 \sigma$ c.l.) obtidos pelo experimento Planck [25].

Na Figura (5.4), adotando de novo os vínculos do Planck para $\Omega_{\chi} h^{2}$, mostramos como a seção de choque $\langle\sigma v\rangle$ depende do parâmetro $\beta$. Como regra geral, para um dado $\Omega_{\chi} h^{2}$, vemos que o valor de $\langle\sigma v>$ cai quando os valores de $\beta$ aumentam. 


\subsection{Produção de WIMPs no Modelo LJO}

Agora analisaremos como a abundância de uma relíquia cósmica é modificada no caso do LJO, ou seja, $\frac{\Gamma_{\chi}}{H}=3 \alpha \frac{H_{0}^{2}}{H^{2}}$. Nesse modelo, a criação de matéria é desprezível no começo do Universo, como pode ser visto pela Figura (3.6). Isso significa que a equação geral de evolução (5.7)

$$
\frac{d Y}{d x}+\frac{\Gamma_{\gamma}-\Gamma_{\chi}}{H x\left(1-\frac{\Gamma_{\gamma}}{3 H}\right)} Y=-\frac{s<\sigma v>}{H x\left(1-\frac{\Gamma_{\gamma}}{3 H}\right)}\left(Y^{2}-Y_{c}^{2}\right)
$$

pode ser simplificada.

Assumiremos que a taxa de criação de radiação é muito menor que a taxa de criação de partículas de matéria escura e a taxa de expansão do Universo, ou seja, $\Gamma_{\gamma} \ll \Gamma_{\chi}$ e $\Gamma_{\gamma} \ll 3 H$. Com isso, até o freeze-out a equação é

$$
\frac{d Y}{d x}=-\frac{s<\sigma v>}{H x}\left(Y^{2}-Y_{c}^{2}\right)
$$

que tem a forma da equação de evolução usual [21, 22].

Após o freeze-out, a equação de balanço para a relíquia já desacoplada do banho térmico é

$$
\dot{n}+3 H n=n \Gamma_{\chi}
$$

Fazendo a mesma troca de variáveis que na seção anterior, ou seja, definindo $Y=\frac{n}{s}$, ficamos com

$$
\dot{Y}=\Gamma_{\chi} Y
$$

Após a era da radiação, entramos na era da matéria e a lei de evolução para uma componente não-relativística, que já não sofre mais influência do banho térmico, é

$$
\frac{\dot{T}}{T}=-2\left(H+\frac{\Gamma_{\chi}}{3}\right)
$$

Introduzindo a quantidade $x=\frac{m}{T}$, temos

$$
\frac{d Y}{d x}=\frac{\Gamma_{\chi}}{2 H x\left(1-\frac{\Gamma_{\chi}}{3 H}\right)} Y=\frac{3 \alpha}{2 x\left(\frac{H^{2}}{H_{0}^{2}}-\alpha\right)} Y .
$$


Das Equações (3.46) e (4.37), pode-se mostrar que a era da matéria é regida por

$$
\frac{H^{2}}{H_{0}^{2}}=\left(\frac{x_{0}}{x}\right)^{3 / 2}
$$

Com isso, a equação de evolução após o freeze-out fica

$$
\frac{d Y}{d x}=\frac{3 \alpha}{2 x\left[\left(\frac{x_{0}}{x}\right)^{3 / 2}-\alpha\right]} Y
$$

Fazendo a troca de variáveis $u=\frac{x}{x_{0}}$, ficamos com a forma final da equação:

$$
\frac{d Y}{d u}=\frac{3 \alpha \sqrt{u}}{2\left[1-\alpha u^{3 / 2}\right]} Y .
$$

Calculamos o valor de $Y_{f}$ no freeze-out através da Equação (5.20). Utilizando o valor encontrado, evoluímos a abundância da relíquia até hoje, $x_{0}$ ou $u=1$. A equação acima tem solução analítica dada por

$$
Y(u)=Y_{f} \frac{\left(\alpha u_{f}^{3 / 2}-1\right)}{\alpha u^{3 / 2}-1}
$$

em que $u_{f}=\frac{x_{f}}{x_{0}}$ é o valor de $u$ no freeze-out.

Na Figura (5.5), mostramos os resultados. Assim como no caso anterior, escolhemos um WIMP de massa $m=100 \mathrm{GeV}$. Lembrando que a densidade do WIMP hoje é $\rho_{\chi, 0}=$ $m_{\chi} n_{\chi, 0}=m_{\chi} s_{0} Y(u=1)$ e usando $s_{0}=2900 \mathrm{~cm}^{-3}$, calculamos o valor do parâmetro de densidade, dado por

$$
\Omega_{\chi} h^{2}=\frac{\rho_{\chi, 0}}{\rho_{c, 0}} h^{2}
$$

A curva vermelha mostra a abundância de uma relíquia com massa $m=100 \mathrm{GeV}$ e seção de choque $\left\langle\sigma v>=1.65 \times 10^{-9} \mathrm{GeV}^{-2}\right.$ para o caso usual (sem criação de matéria). Esse valor foi escolhido para que a abundância final esteja dentro do intervalo fornecido pelo experimento Planck (região em cinza, que está compreendida entre $0.1165 \leq \Omega_{\chi} h^{2} \leq$ $0.1227)$.

A curva preta mostra a evolução para o caso LJO. Para uma massa de $m=100 \mathrm{GeV}$, a seção de choque para que a abundância hoje esteja dentro do intervalo fornecido pelo Planck é $\langle\sigma v\rangle=3.71 \times 10^{-8} \mathrm{GeV}^{-2}$. Como referência, mostramos a curva em azul, que é o caso usual com esses valores para a massa e seção de choque. Vemos que a presença de 


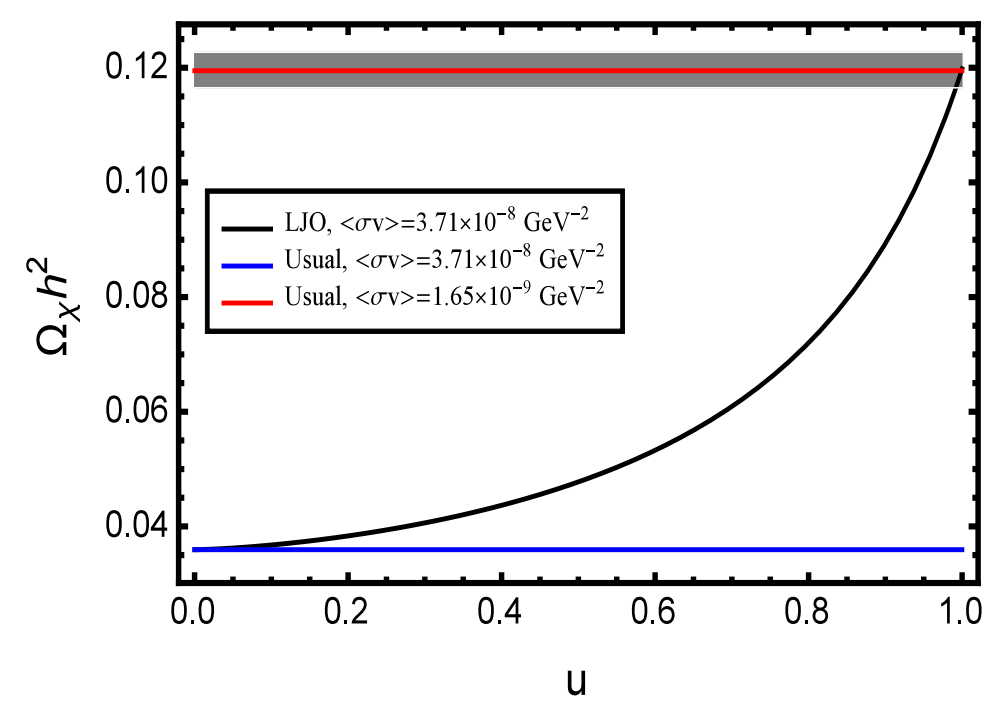

Figura 5.5: $\Omega_{\chi} h^{2}$ em função de $u$ para o modelo LJO.

uma criação gravitacional de partículas implicaria um aumento de uma ordem de grandeza no valor esperado para a seção de choque. O valor de $x_{0}=10^{24}$ é baseado no trabalho de $[77]$.

Na Figura (5.6), mostramos a evolução da diferença percentual entre o modelo LJO e o caso usual sem criação de matéria. A linha vertical vermelha representa o momento em que a taxa de criação de matéria se iguala à taxa de expansão do Universo (após esse instante, a taxa de criação se torna cada vez maior em relação à taxa de expansão do Universo). Esse é o ponto correspondente ao redshift de transição presente na Figura $(3.6)$.

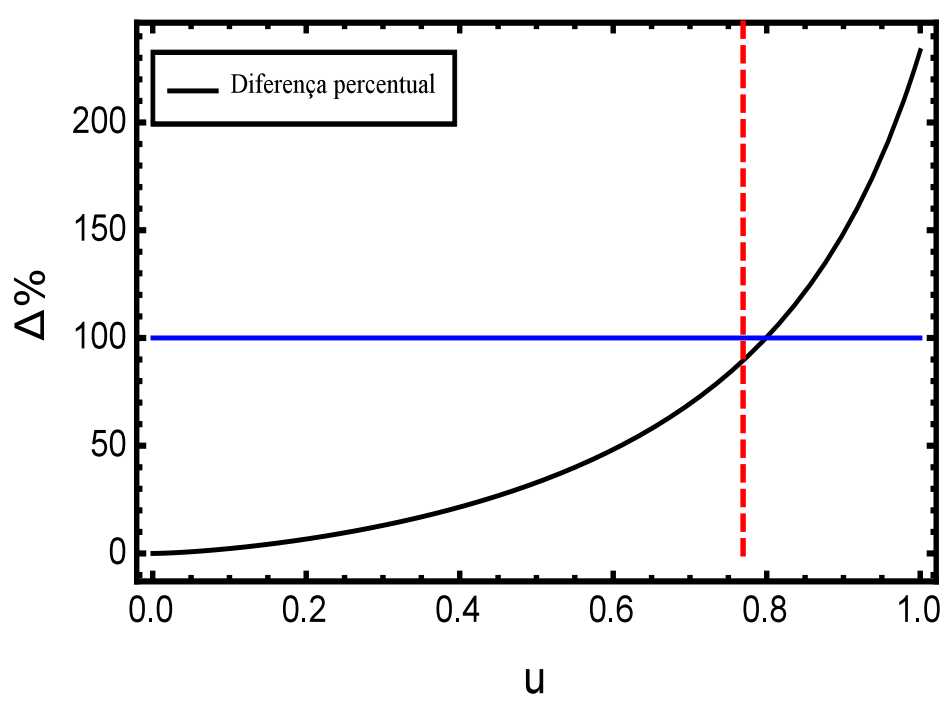

Figura 5.6: Diferença percentual entre modelo LJO para o caso usual sem criação de matéria em função de $u$. 


\section{Capítulo 6}

\section{Conclusão e Perspectivas}

No final do século passado, a Cosmologia passou por uma revolução: descobriu-se que, diferentemente do que se esperava, a expansão do Universo é acelerada. O candidato mais natural para esse fato é a constante cosmológica, dando ao modelo $\Lambda$ CDM o lugar de modelo padrão da Cosmologia atual. Contudo, tal modelo sofre com sintomas graves: falta de um mecanismo capaz de explicar a discrepância entre observação e teoria no valor da energia do vácuo associada à energia escura e o problema da coincidência cósmica. Admitindo-se a Relatividade Geral, modelos que possuam uma pressão negativa podem ser considerados alternativas ao $\Lambda$ CDM. Dentro desse contexto, modelos com criação gravitacional de partículas vêm tomando cada vez mais visibilidade.

Nesta tese, fizemos uma breve revisão da Cosmologia moderna. Apresentamos algumas ferramentas da Relatividade Geral que foram usadas para construir o Modelo Padrão da Cosmologia. Com o objetivo de ilustrar alguns exemplos, apresentamos modelos cosmológicos simples.

No Capítulo 2, fizemos uma revisão da teoria cinética relativística. O maior objetivo desse capítulo foi entender como a equação de Boltzmann na métrica de FLRW é obtida. A partir da equação de Botlzmann, obtivemos a equação de evolução para relíquias cósmicas.

No Capítulo 3, foi exposta uma explicação alternativa ao problema do setor escuro. Nessa nova abordagem, matéria escura é criada a partir do campo gravitacional. O efeito dinâmico que esse processo causa no Universo é a presença de uma pressão negativa, podendo assim explicar a expansão acelerada do Universo. A energia escura, nesse contexto, faz-se desnecessária. Portanto, poderíamos reduzir o setor escuro a uma componente.

Fizemos no Capítulo 4 nossa primeira contribuição original ao problema de criação de 
matéria [17]. Propusemos um termo fenomenológico de origem não-colisional à equação de Boltzmann covariante capaz de reproduzir todos os resultados da formulação termodinâmica fora de equilíbrio. O objetivo principal desse trabalho era dar uma justificativa cinética para a produção de partículas através do campo gravitacional. Com o termo proposto, fomos capazes de reobter as equações de balanço e a lei de evolução da temperatura. Dessa forma, resolvemos um problema que estava em aberto desde 1992 [59].

Nossa segunda contribuição original se encontra no Capítulo 5. Após obter a forma correta da generalização da equação de Boltzmann, aplicamos o formalismo para estudar como a criação de partículas pode afetar a equação de evolução de relíquias cósmicas. Nossos resultados do artigo [18] podem ser resumidos nos seguintes itens:

1. Para todos valores aceitáveis para $\beta$, o cenário qualitativo básico é mantido e a abundância final satura quando a aniquilação se torna desprezível. A abundância final calculada é modificada na presença de uma produção gravitacional de partículas. Ver Figura (5.1).

2. Para a taxa de criação adotada $\left(\Gamma_{\chi}=3 \beta H\right)$, a nova equação de evolução apenas se diferencia por um fator $1 /(1-\beta)$ quando comparada à equação usual. Ver Equação $(5.11)$.

3. A diferença percentual das relíquias na presença da criação de matéria pode ser mudada por fatores de (10\% a 30\%) dependendo dos valores assumidos para $\beta$. Ver Figura (5.2)

4. A densidade atual da matéria escura e a seção de choque de aniquilação são fortemente dependentes do parâmetro $\beta$. Para todos os valores de $\beta$, a seção de choque deve ser menor que a do caso usual (quando $\beta=0$ ) para que se acomodem os recentes resultados do satélite Planck. Ver Figuras (5.3) e (5.4).

Outra aplicação, ainda não submetida a publicação, focaliza no cálculo de abundância de WIMPs para o chamado modelo LJO. Como sabemos, tal modelo pode ser considerado uma alternativa viável para o modelo $\Lambda$ CDM [3]. Nesse modelo, a presença de criação de partículas de matéria escura implica um aumento de uma ordem de grandeza da seção de choque de um WIMP. Ver Figura (5.5).

Para trabalhos futuros, pretendemos aprofundar nosso entendimento do processo de criação gravitacional de partículas. Para isso, buscaremos formulações do ponto de vista 
da teoria cinética quântica ou mesmo em teorias quânticas em espaços curvos. Também buscaremos consequências desses modelos em testes cosmológicos que envolvam formalismos cinéticos como, por exemplo, análise da radiação cósmica de fundo. 


\section{Apêndice A}

\section{Dedução alternativa da Equação de}

\section{Boltzmann}

Nessa seção deduziremos a equação de Boltzmann na métrica FLRW de forma alternativa àquela apresentada no texto. Aqui serão seguidos os passos de [55].

A variação da função distribuição é

$$
\frac{d f\left(x^{\mu}, P^{\mu}\right)}{d \lambda}=P^{\mu} \frac{\partial f}{\partial x^{\mu}}+\frac{\partial f}{\partial P^{\mu}} \frac{d P^{\mu}}{d \lambda} .
$$

Através da equação da geodésica,

$$
\frac{d P^{\mu}}{d \lambda}=-\Gamma_{\alpha \beta}^{\mu} P^{\alpha} P^{\beta}
$$

e dos símbolos de Christoffel não nulos, temos as seguintes relações:

$$
\frac{d P^{0}}{d \lambda}=-P^{i} P^{j} \Gamma_{i j}^{0}=-\delta_{i j} P^{i} P^{j} \dot{a} a=-P^{2} \dot{a} a
$$

$\mathrm{e}$

$$
\frac{d P^{i}}{d \lambda}=-2 \Gamma^{i}{ }_{0 j} P^{0} P^{j}-\Gamma_{j k}^{i} P^{j} P^{k}=-2 \frac{\dot{a}}{a} \delta_{j}^{i} P^{0} P^{j}=-2 \frac{\dot{a}}{a} P^{0} P^{i}
$$

Agora, usando as conexões da métrica FLRW para um Universo plano, ficamos com

$$
\frac{d f\left(x^{\mu}, P^{\mu}\right)}{d \lambda}=P^{0} \frac{\partial f}{\partial t}-2 \frac{\dot{a}}{a} P^{0} P^{i} \frac{\partial f}{\partial P^{i}}-\dot{a} a P^{2} \frac{\partial f}{\partial P^{0}},
$$

em que foi assumido que a função distribuição não depende das posições. 
Definimos a função [55]

$$
\hat{f}(P, t)=\int \delta\left[P^{0}-\left(P^{2} a^{2}+m^{2} c^{2}\right)^{1 / 2}\right] f\left(\boldsymbol{P}, P^{0}, t\right) d P^{0}
$$

Calculando a derivada temporal da expressão acima temos

$$
\begin{aligned}
\frac{\partial \hat{f}}{\partial t}= & \int \frac{\partial f}{\partial t} \delta\left[P^{0}-\left(P^{2} a^{2}+m^{2} c^{2}\right)^{1 / 2}\right] d P^{0}+\int f \frac{\partial}{\partial t} \delta\left[P^{0}-\left(P^{2} a^{2}+m^{2} c^{2}\right)^{1 / 2}\right] d P^{0} \\
= & \int \frac{\partial f}{\partial t} \delta\left[P^{0}-\left(P^{2} a^{2}+m^{2} c^{2}\right)^{1 / 2}\right] d P^{0}+ \\
& \left.\frac{\partial P^{0}}{\partial t}\right|_{P^{0}=\left(P^{2} a^{2}+m^{2} c^{2}\right)^{1 / 2}} \int f \frac{\partial}{\partial P^{0}} \delta\left[P^{0}-\left(P^{2} a^{2}+m^{2} c^{2}\right)^{1 / 2}\right] d P^{0} \\
= & \int \frac{\partial f}{\partial t} \delta\left[P^{0}-\left(P^{2} a^{2}+m^{2} c^{2}\right)^{1 / 2}\right] d P^{0}-\left.\dot{a} a \frac{\partial f}{\partial P^{0}} \frac{P^{2}}{P^{0}}\right|_{P^{0}=\left(P^{2} a^{2}+m^{2} c^{2}\right)^{1 / 2}}
\end{aligned}
$$

em que foram usadas as seguintes propriedades:

$$
\begin{gathered}
\frac{\partial}{\partial t} \delta\left[P^{0}-\left(P^{2} a^{2}+m^{2} c^{2}\right)^{1 / 2}\right]=\left.\frac{\partial P^{0}}{\partial t}\right|_{P^{0}=\left(P^{2} a^{2}+m^{2} c^{2}\right)^{1 / 2}} \frac{\partial}{\partial P^{0}}\left[P^{0}-\left(P^{2} a^{2}+m^{2} c^{2}\right)^{1 / 2}\right] \\
\left.\frac{\partial P^{0}}{\partial t}\right|_{P^{0}=\left(P^{2} a^{2}+m^{2} c^{2}\right)^{1 / 2}}=\dot{a} a \frac{P^{2}}{P^{0}} \\
\int \delta^{\prime}(x) \phi(x) d x=-\int \delta(x) \phi^{\prime}(x) d x
\end{gathered}
$$

Faremos uma integral na camada de massa

$$
\begin{aligned}
& \int \frac{d f}{d \lambda} \frac{1}{P^{0}} \delta\left[P^{0}-\left(P^{2} a^{2}+m^{2} c^{2}\right)^{1 / 2}\right] d P^{0}= \\
& \int \frac{\partial f}{\partial t} \delta\left[P^{0}-\left(P^{2} a^{2}+m^{2} c^{2}\right)^{1 / 2}\right] d P^{0}-\left.\dot{a} a \frac{\partial f}{\partial P^{0}} \frac{P^{2}}{P^{0}}\right|_{P^{0}=\left(P^{2} a^{2}+m^{2} c^{2}\right)^{1 / 2}}-2 \frac{\dot{a}}{a} P^{i} \frac{\partial \hat{f}}{\partial P^{i}} \\
& \int \frac{d f}{d \lambda} \frac{1}{P^{0}} \delta\left[P^{0}-\left(P^{2} a^{2}+m^{2} c^{2}\right)^{1 / 2}\right] d P^{0}=\frac{\partial \hat{f}}{\partial t}-2 \frac{\dot{a}}{a} P^{i} \frac{\partial \hat{f}}{\partial P^{i}}
\end{aligned}
$$

Com isso, escreveremos o operador de Liouville, $L(f)$, na métrica de FLRW,

$$
L(f)=\frac{\partial f}{\partial t}-2 \frac{\dot{a}}{a} P^{i} \frac{\partial f}{\partial P^{i}}
$$

em que abandonamos o "chapéu" por conveniência de notação, mas sempre lembrando que agora todas as quantidades já estão na camada de massa. A equação acima é a mesma 
que a Eq (2.71) sem o termo de colisão. 


\section{Apêndice B}

\section{Dedução do termo colisional da}

\section{equação de evolução}

Nessa seção, seguindo os passos de [21], vamos determinar o lado direito da equação de evolução para relíquias cósmicas. O termo colisional para processos do tipo $\psi+a+b+$ $\cdots \longleftrightarrow i+j+\cdots$ é dado por

$$
\begin{aligned}
\frac{g}{(2 \pi)^{3}} \int \mathcal{C}[f] \frac{d^{3} p_{\psi}}{E_{\psi}}= & -\int d \Pi_{\psi} d \Pi_{a} d \Pi_{b} \cdots d \Pi_{i} d \Pi_{j} \cdots \\
& \times(2 \pi)^{4} \delta^{4}\left(p_{\psi}+p_{a}+p_{b} \cdots-p_{i}-p_{j} \cdots\right) \\
& \times\left[|\mathcal{M}|_{\psi+a+b+\cdots \rightarrow i+j+\cdots}^{2} f_{a} f_{b} \cdots f_{\psi}\left(1 \pm f_{i}\right)\left(1 \pm f_{j}\right) \cdots\right. \\
& \left.-|\mathcal{M}|_{i+j+\cdots \rightarrow \psi+a+b+\cdots}^{2} f_{i} f_{j} \cdots\left(1 \pm f_{a}\right)\left(1 \pm f_{b}\right) \cdots\left(1 \pm f_{\psi}\right)\right],
\end{aligned}
$$

em que $f_{i}, f_{j}, f_{a}, f_{b}, \cdots$ são as funções de distribuição para as espécies $i, j, \cdots, a, b$ e $f_{\psi}$ é a função de distribuição para espécie cuja evolução estamos estudando. Aqui $(+)$ se aplica para bósons e (-) se aplica para férmions. O volume de espaço de fase é

$$
d \Pi \equiv g \frac{1}{(2 \pi)^{3}} \frac{d^{3} p}{2 E}
$$

em que $g$ conta os graus de liberdade internos. O delta quadridimensional garante a conservação de energia e momento. A matriz $|\mathcal{M}|_{i+j+\cdots \rightarrow \psi+a+b+\cdots}^{2}$ contém informações sobre processos $i+j+\cdots \rightarrow \psi+a+b+\cdots$, para os quais, quando se assume invariância 
temporal, temos a propriedade

$$
|\mathcal{M}|_{i+j+\cdots \rightarrow \psi+a+b+\cdots}^{2}=|\mathcal{M}|_{\psi+a+b+\cdots \rightarrow i+j+\cdots}^{2} \equiv|\mathcal{M}|^{2}
$$

Usando a estatística de Maxwell-Boltzmann para todas espécies, ficamos com a equação de evolução

$$
\begin{aligned}
\dot{n}_{\psi}+ & 3 H n_{\psi}=-\int d \Pi_{\psi} d \Pi_{a} d \Pi_{b} \cdots d \Pi_{i} d \Pi_{j} \cdots(2 \pi)^{4}|\mathcal{M}|^{2} \\
& \times \delta^{4}\left(p_{i}+p_{j} \cdots-p_{\psi}-p_{a}-p_{b} \cdots\right)\left[f_{a} f_{b} \ldots f_{\psi}-f_{i} f_{j} \cdots\right] .
\end{aligned}
$$

Vamos estudar o caso em que o número de partículas de uma espécie estável ( $\psi$ e sua antipartícula $\bar{\psi}$ ) muda apenas através de processos de aniquilações e destruição por pares

$$
\psi \bar{\psi} \longleftrightarrow X \bar{X}
$$

em que $X$ representa todas as espécies em que $\psi$ pode se aniquilar.

Assumiremos que $X$ possui potencial químico nulo. Isso se justifica pelo fato de que as partículas $X$ terão um forte contato térmico com o banho relativístico. Com isso a função de distribuição para a espécie $X$ é

$$
\begin{aligned}
& f_{X}=\exp \left(-\frac{E_{X}}{T}\right) \\
& f_{\bar{X}}=\exp \left(-\frac{E_{\bar{X}}}{T}\right) .
\end{aligned}
$$

A conservação de energia implica que

$$
f_{X} f_{\bar{X}}=\exp \left[-\left(E_{X}+E_{\bar{X}}\right) / T\right]=\exp \left[-\left(E_{\psi}+E_{\bar{\psi}}\right) / T\right]=f_{\psi}^{\mathrm{Eq}} f_{\bar{\psi}}^{\mathrm{Eq}}
$$

Com isso, temos que

$$
\left[f_{\psi} f_{\bar{\psi}}-f_{X} f_{\bar{X}}\right]=\left[f_{\psi} f_{\bar{\psi}}-f_{\psi}^{\mathrm{Eq}} f_{\bar{\psi}}^{\mathrm{Eq}}\right]
$$

Assim, a equação de evolução pode ser escrita em termos de $n_{\psi}$ e $n_{\psi}^{\text {Eq. }}$

$$
\frac{d n_{\psi}}{d t}+3 H n_{\psi}=-\left\langle\sigma_{\psi \bar{\psi} \rightarrow X \bar{X}}|v|\right\rangle\left[n_{\psi}^{2}-\left(n_{\psi}^{\mathrm{Eq}}\right)^{2}\right]
$$


em que definimos a média termal da seção de choque de aniquilação como

$$
\begin{aligned}
\left\langle\sigma_{\psi \bar{\psi}} \rightarrow X \bar{X}|v|\right\rangle \equiv\left(n_{\psi}^{\mathrm{Eq}}\right)^{-2} \int d \Pi_{\psi} d \Pi_{\bar{\psi}} d \Pi_{X} d \Pi_{\bar{X}}(2 \pi)^{4} \\
\quad \times \delta^{4}\left(p_{\psi}+p_{\bar{\psi}}-p_{X}-p_{\bar{X}}\right)|\mathcal{M}|^{2} \exp \left(-E_{\psi} / T\right) \exp \left(-E_{\bar{\psi}} / T\right) .
\end{aligned}
$$




\section{Apêndice $\mathrm{C}$}

\section{Equação de Boltzmann em}

\section{momentos comóveis}

Na Seção 2.4.1 calculamos as equações de balanço utilizando momentos físicos. Nessa seção iremos obter os mesmos resultados usando a equação de Boltzmann em momentos comóveis (2.71) sem colisões, ou seja

$$
\frac{\partial f}{\partial t}-2 H p^{i} \frac{\partial f}{\partial p^{i}}=0
$$

A condição de camada de massa nos dá as seguintes propriedades:

$$
E=\sqrt{a^{2} p^{2}+m^{2}}, \frac{\partial E}{\partial t}=a \dot{a} \frac{p^{2}}{E}, \frac{\partial E}{\partial p}=a^{2} \frac{p}{E} .
$$

Usando as definições usuais das quantidades macroscópicas

$$
\begin{aligned}
N^{\mu} & =\int f p^{\mu} \sqrt{g} \frac{d^{3} p}{p^{0}}=\int f p^{\mu} a^{3} \frac{d^{3} p}{p^{0}} \\
S^{\mu} & =-\int\left(f \ln \left(\frac{f h^{3}}{g}\right)-f\right) p^{\mu} \sqrt{g} \frac{d^{3} p}{p^{0}} \\
& =-\int\left(f \ln \left(\frac{f h^{3}}{g}\right)-f\right) p^{\mu} a^{3} \frac{d^{3} p}{p^{0}}, \\
T^{\mu \nu} & =\int f p^{\mu} p^{\nu} \sqrt{g} \frac{d^{3} p}{p^{0}}=\int f p^{\mu} p^{\nu} a^{3} \frac{d^{3} p}{p^{0}},
\end{aligned}
$$

Observando que, devido à isotropia do espaço, as únicas componentes não nulas de $N^{\mu}$ e 
$S^{\mu}$ são dadas por

$$
\begin{aligned}
& N^{0}=n=\int f a^{3} d^{3} p, \\
& S^{0}=s=-\int(f \ln f-f) a^{3} d^{3} p .
\end{aligned}
$$

Agora, com a ajuda do operador de Boltzmann (C.1), o divergente dos fluxos termodinâmicos podem ser calculados.

- Fluxo de Partículas

$$
\begin{aligned}
N_{; \mu}^{\mu} & \equiv \frac{1}{a^{3}} \frac{\partial}{\partial x^{\mu}}\left(a^{3} \int f p^{\mu} a^{3} \frac{d^{3} p}{p^{0}}\right)=\frac{1}{a^{3}} \frac{\partial}{\partial t}\left(a^{6} \int f d^{3} p\right) \\
& =6 H n+2 a^{3} H \int p \frac{\partial f}{\partial p} d^{3} p,
\end{aligned}
$$

em que a Eq. (C.1) foi usada no último passo. Inserindo o resultado da integração por partes, ficamos com

$$
N_{; \mu}^{\mu}=0
$$

- Fluxo de Entropia

$$
\begin{aligned}
S_{; \mu}^{\mu} & \equiv-\frac{1}{a^{3}} \frac{\partial}{\partial x^{\mu}}\left(a^{3} \int(f \ln f-f) p^{\mu} a^{3} \frac{d^{3} p}{p^{0}}\right) \\
& =-\frac{1}{a^{3}} \frac{\partial}{\partial t}\left(a^{6} \int(f \ln f-f) d^{3} p\right) \\
& =6 s H-\int \frac{\partial f}{\partial t} \ln f d^{3} p \\
& =6 s H-2 a^{3} H \int p \frac{\partial f}{\partial p} \ln f d^{3} p .
\end{aligned}
$$

A última integral já foi calculada e quando substituída, obtemos

$$
S_{; \mu}^{\mu}=0
$$

- Tensor Energia-Momento

Vamos agora calcular a projeção do divergente do tensor de energia-momento na direção de $u_{\mu}$ : 


$$
u_{\mu} T_{; \nu}^{\mu \nu} \equiv u_{\mu}\left[\frac{1}{a^{3}} \frac{\partial}{\partial x^{\nu}}\left(a^{3} T^{\mu \nu}\right)+\Gamma_{\alpha \beta}^{\mu} T^{\alpha \beta}\right],
$$

o qual somaremos sobre os índices repetidos; usando os símbolos de Christoffel não nulos temos

$$
\begin{aligned}
u_{\mu} T_{; \nu}^{\mu \nu} & \equiv \frac{1}{a^{3}} \frac{\partial}{\partial t}\left(a^{3} T^{00}\right)+\Gamma_{i j}^{0} T^{i j} \\
& =\frac{1}{a^{3}} \frac{\partial}{\partial t}\left(a^{6} \int f E d^{3} p\right)+3 \frac{\dot{a}}{a} P \\
& =6 H \rho+3 H P+a^{3} \int \frac{\partial E}{\partial t} f d^{3} p+a^{3} \int E \frac{\partial f}{\partial t} d^{3} p \\
& =6 H \rho+3 H P+a^{4} \dot{a} \int f \frac{p^{2}}{E} d^{3} p+a^{3} 2 H \int E p \frac{\partial f}{\partial p} d^{3} p \\
& =6 H \rho+3 H P+3 H P+a^{3} 2 H \int E p \frac{\partial f}{\partial p} d^{3} p \\
& =0 .
\end{aligned}
$$




\section{Referências Bibliográficas}

[1] E. Hubble. A relation between distance and radial velocity among extra-galactic nebulae. Proc.Nat.Acad.Sci., 15:168-173, 1929.

[2] N. Suzuki et al. The Hubble Space Telescope Cluster Supernova Survey: V. Improving the Dark Energy Constraints Above $z>1$ and Building an Early-Type-Hosted Supernova Sample. Astrophys. J., 746:85, 2012.

[3] J.A.S. Lima, J.F. Jesus, and F.A. Oliveira. CDM Accelerating Cosmology as an Alternative to LCDM model. JCAP, 1011:027, 2010.

[4] A. Einstein. The Foundation of the General Theory of Relativity. Annalen Phys., 49:769-822, 1916.

[5] A. Einstein. Cosmological Considerations in the General Theory of Relativity. Sitzungsber.Preuss.Akad.Wiss.Berlin (Math.Phys.), 1917:142-152, 1917.

[6] A. Friedman. On the curvature of space. General Relativity and Gravitation, 31(12):1991-2000, 1999.

[7] A. Friedmann. On the possibility of a world with constant negative curvature of space. General Relativity and Gravitation, 31(12):2001-2008, 1999.

[8] A. G. Lemaître. A homogeneous universe of constant mass and increasing radius accounting for the radial velocity of extra-galactic nebulae. Monthly Notices of the Royal Astronomical Society, 91(5):483-490, 1931.

[9] S. Perlmutter et al. Measurements of Omega and Lambda from 42 high redshift supernovae. Astrophys.J., 517:565-586, 1999.

[10] Adam G. Riess et al. Observational evidence from supernovae for an accelerating universe and a cosmological constant. Astron.J., 116:1009-1038, 1998. 
[11] M. Roos. Dark Matter: The evidence from astronomy, astrophysics and cosmology. arXiv:1001.0316, 2010.

[12] M. J. Mortonson, D. H. Weinberg, and M. White. Dark Energy: A Short Review. arXiv:1401.0046, 2013.

[13] J. A. S. Lima. Alternative dark energy models: an overview. Brazilian Journal of Physics, 34:194 - 200, 032004.

[14] Ya. B. Zeldovich. JETP Lett., 12:307, 1970.

[15] I. Prigogine, J. Geheniau, E. Gunzig, and P. Nardone. Thermodynamics and cosmology. Gen.Rel.Grav., 21:767-776, 1989.

[16] J.A.S. Lima and A.S.M. Germano. On the Equivalence of matter creation in cosmology. Phys.Lett., A170:373-378, 1992.

[17] J. A. S. Lima and I. Baranov. Gravitationally Induced Particle Production: Thermodynamics and Kinetic Theory. Phys. Rev., D90(4):043515, 2014.

[18] I. Baranov and J. A. S. Lima. Gravitationally Induced Particle Production and its Impact on the WIMP Abundance. arXiv, 1505.02743, 2015.

[19] S. Weinberg. Gravitation and Cosmology, Principles and applications of the General Theory of Relativity. John Wiley \& Sons, 1972.

[20] S. Weinberg. Cosmology. Oxford University Press, 2008.

[21] E. W. Kolb and M. Turner. The Early Universe. Westview Press, 1990.

[22] S. Dodelson. Modern Cosmology. Elsevier, 2003.

[23] R. J. A. Lambourne. Relativity, Gravitation and Cosmology. The Open University, 2010.

[24] B. Ryden. Introduction to cosmology. Pearson Addison-Wesley, 2003.

[25] P. A. R. Ade et al. Planck 2015 results. XIII. Cosmological parameters. arXiv:1502.01589, 2015.

[26] S. Serjeant. Observational Cosmology. The Open University, 2010. 
[27] T. Padmanabhan. Cosmological constant: The Weight of the vacuum. Phys. Rept., 380:235-320, 2003.

[28] P. J. E. Peebles and Bharat Ratra. The cosmological constant and dark energy. Rev. Mod. Phys., 75:559-606, Apr 2003.

[29] M Özer and M. O. Taha. A possible solution to the main cosmological problems. Physics Letters B, 171(4):363 - 365, 1986.

[30] J. C. Carvalho, J. A. S. Lima, and I. Waga. Cosmological consequences of a timedependent $\Lambda$ term. Phys. Rev. D, 46:2404-2407, Sep 1992.

[31] J. A. S. Lima and J. M. F. Maia. Deflationary cosmology with decaying vacuum energy density. Phys. Rev. D, 49:5597-5600, May 1994.

[32] J. A. S. Lima and M. Trodden. Decaying vacuum energy and deflationary cosmology in open and closed universes. Phys. Rev., D53:4280-4286, 1996.

[33] J. M. Overduin and F. I. Cooperstock. Evolution of the scale factor with a variable cosmological term. Phys. Rev. D, 58:043506, Jul 1998.

[34] J. M. F. Maia and J. A. S. Lima. Scalar field description of decaying Lambda cosmologies. Phys. Rev., D65:083513, 2002.

[35] J. S. Alcaniz and J. A. S. Lima. Interpreting cosmological vacuum decay. Phys. Rev., D72:063516, 2005.

[36] S. Basilakos, M. Plionis, and J. A. S. Lima. Confronting Dark Energy Models using Galaxy Cluster Number Counts. Phys. Rev., D82:083517, 2010.

[37] M. S. Turner and M. White. Cdm models with a smooth component. Phys. Rev. D, 56:R4439-R4443, Oct 1997.

[38] J. S. Alcaniz and J. A. S. Lima. New limits on $\Omega_{\Lambda}$ and $\Omega_{m}$ from old galaxies at high redshift. Astrophys. J., 521:L87, 1999.

[39] J. A. S. Lima. Alternative dark energy models: An Overview. Braz. J. Phys., 34:194-200, 2004. 
[40] A. Kamenshchik, U. Moschella, and V. Pasquier. An alternative to quintessence. Physics Letters B, 511(2,4):265 - 268, 2001.

[41] M. Makler, S. Quinet de Oliveira, and I. Waga. Observational constraints on chaplygin quartessence: Background results. Phys. Rev. D, 68:123521, Dec 2003.

[42] J. A. S. Lima, J. V. Cunha, and J. S. Alcaniz. Constraining the dark energy with galaxy clusters x-ray data. Phys. Rev., D68:023510, 2003.

[43] J. S. Alcaniz and J. A. S. Lima. Measuring the chaplygin gas equation of state from angular and luminosity distances. Astrophys. J., 618:16, 2005.

[44] J. L. Feng. Dark Matter Candidates from Particle Physics and Methods of Detection. Ann. Rev. Astron. Astrophys., 48:495-545, 2010.

[45] F. Jüttner. Das Maxwellsche Gesetz der Geschwindigkeitsverteilung in der Relativtheorie. Ann. Physik und Chemie, 34:856-882, 1911.

[46] F. Jüttner. Die relativistische Quantentheorie des idealen Gases. Zeitschr. Physik, 47:542-566, 1928.

[47] A. G. Walker. The boltzmann equations in general relativity. Proceedings of the Edinburgh Mathematical Society (Series 2), 4:238-253, 41936.

[48] A. Lichnerowicz and R. Marrot. Propriétés statistiques des ensembles de particules en relativité restreinte. C. R. Acad. Sci. Paris, 210:759-761, 1940.

[49] R. Marrot. Sur l'équation intégrodifferentielle de boltzmann. J. Math. Pures et Appl., 25:93-113, 1940.

[50] J. Ehlers. Contributions to the relativistic mechanics of continuous media. General Relativity and Gravitation, 25(12):1225-1266, 1993.

[51] G. E. Tauber and J. W. Weinberg. Internal state of a gravitating gas. Phys. Rev., 122:1342-1365, May 1961.

[52] N. A. Chernikov. The relativistic gas in the gravitational field. Acta Physica Polonica, 23:629-645, 1963. 
[53] C. Cercignani and G. M. Kremer. The Relativistic Boltzmann Equation: Theory and Applications. Birkhäuser Verlag, 2002.

[54] S. R. de Groot, W. A. van Leeuwen, and Ch. G. van Weert. Relativistic Kinetic Theory - Principles and Applications. North-Holland Publishing Company, 1980.

[55] J. Bernstein. Kinetic Theory in Expanding Universe. University Press, 1988.

[56] L. Parker. Particle creation in expanding universes. Phys. Rev. Lett., 21:562-564, Aug 1968.

[57] S. A. Fulling, L. Parker, and B. L. Hu. Conformal energy-momentum tensor in curved spacetime: Adiabatic regularization and renormalization. Phys. Rev. D, 10:39053924, Dec 1974.

[58] N. D. Birrell and P. C. Davies. Quantum Fields in Curved Space. University Press, 1982.

[59] M.O. Calvao, J.A.S. Lima, and I. Waga. On the thermodynamics of matter creation in cosmology. Phys.Lett., A162:223-226, 1992.

[60] R. Silva, J.A.S. Lima, and M.O. Calvao. Temperature evolution law of imperfect relativistic fluids. Gen.Rel.Grav., 34:865-875, 2002.

[61] J.A.S. Lima, F.E. Silva, and R.C. Santos. Accelerating Cold Dark Matter Cosmology $\left(\Omega_{\Lambda} \equiv 0\right)$. Class.Quant.Grav., 25:205006, 2008.

[62] J. A. S. Lima, A. S. M. Germano, and L. R. W. Abramo. Frw-type cosmologies with adiabatic matter creation. Phys. Rev. D, 53:4287-4297, Apr 1996.

[63] J.A.S. Lima and J.S. Alcaniz. Flat FRW cosmologies with adiabatic matter creation: Kinematic tests. Astron.Astrophys., 348:1-7, 1999.

[64] G. Steigman, R.C. Santos, and J.A.S. Lima. An Accelerating Cosmology Without Dark Energy. JCAP, 0906:033, 2009.

[65] S. Basilakos and J.A.S. Lima. Constraints on Cold Dark Matter Accelerating Cosmologies and Cluster Formation. Phys.Rev., D82:023504, 2010. 
[66] J.A.S. Lima, S. Basilakos, and F.E.M. Costa. New Cosmic Accelerating Scenario without Dark Energy. Phys.Rev., D86:103534, 2012.

[67] J. P. Mimoso and D. Pavón. Entropy evolution of universes with initial and final de sitter eras. Phys. Rev. D, 87:047302, Feb 2013.

[68] J.A.S. Lima, J.V. Cunha, and J.S. Alcaniz. Constraining the dark energy with galaxy clusters x-ray data. Phys.Rev., D68:023510, 2003.

[69] J. F. Jesus, F. A. Oliveira, S. Basilakos, and J. A. S. Lima. Newtonian perturbations on models with matter creation. Phys. Rev. D, 84:063511, Sep 2011.

[70] R. O. Ramos, M. V. dos Santos, and I. Waga. Matter creation and cosmic acceleration. Phys. Rev. D, 89:083524, Apr 2014.

[71] M. V. dos Santos, I. Waga, and R. O. Ramos. Degeneracy between ccdm and $\Lambda$ CDM cosmologies. Phys. Rev. D, 90:127301, Dec 2014.

[72] J. A. S. Lima, R. C. Santos, and J. V. Cunha. Is $\Lambda$ CDM an effective CCDM cosmology? arXiv:1508.07263, 2015.

[73] J. Triginer, W. Zimdahl, and D. Pavon. Kinetic theory for particle production. Class. Quant. Grav., 13:403-416, 1996.

[74] J.A.S. Lima, A.I. Silva, and S.M. Viegas. Is the radiation temperature redshift relation of the standard cosmology in accordance with the data? Mon.Not.Roy.Astron.Soc., 312:747-752, 2000.

[75] M. Drees, H. Iminniyaz, and M. Kakizaki. Abundance of cosmological relics in lowtemperature scenarios. Phys. Rev., D73:123502, 2006.

[76] G. Steigman, B. Dasgupta, and J. F. Beacom. Precise Relic WIMP Abundance and its Impact on Searches for Dark Matter Annihilation. Phys. Rev., D86:023506, 2012.

[77] C. Armendariz-Picon and J. T. Neelakanta. How cold is cold dark matter? Journal of Cosmology and Astroparticle Physics, 2014(03):049, 2014. 